\title{
Untersuchung von bipolaren Vakuumbögen auf Erste-Wand-Materialien
}

\author{
Diplomarbeit
}

zur Erlangung des akademischen Grades

Diplomingenieur „Dipl.-Ing (FH)“

\section{Technische Hochschule Wildau}

Fachbereich Ingenieurwesen / Wirtschaftsingenieurwesen

Studiengang: Ingenieurwesen

Studienrichtung: Physikalische Technik

eingereicht von: Mathias Marx

geboren am : 16.09.1986

Reg.-Nr.: P06/11/SS2010

eingereicht am: 4. April 2011

Betreuer/Einrichtung:

Dr. rer. nat. P. Siemroth/Arc Precision

Themenstellendes Institut:

Dipl. phys. H. Beyer/TH-Wildau

Arc Precision - Sources Coatings and Analysis GmbH 



\section{Selbständigkeitserklärung}

Ich erkläre hiermit, dass ich die vorliegende Arbeit selbstständig angefertigt und nur die angegeben Quellen und Hilfsmittel verwendet habe.

Datum, Unterschrift 


\section{Inhaltsverzeichnis}

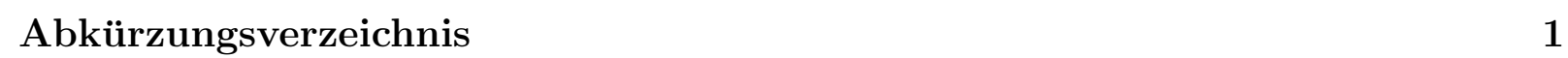

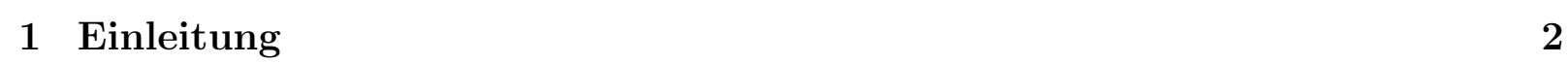

2 Kurzer Abriss des Wissenstandes - Theorie $\quad 4$

$2.1 \quad$ Einführung in den Vakuumbogen . . . . . . . . . . . . . . . . . . . . . 4

2.2 Emission der Ladungsträger . . . . . . . . . . . . . . . . . . . . . . . . . 5

$2.3 \quad$ Quasineutralität, Brennspannung und Bogenstrom . . . . . . . . . . . . . . 6

2.4 Kathodenerosion $\ldots \ldots \ldots \ldots \ldots$

2.5 Brennflecktypen . . . . . . . . . . . . . . . . . . . . . . . . . . . . . . . . . . . . . . . . . .

2.6 Brennfleckbewegung $\ldots \ldots \ldots \ldots$

\begin{tabular}{|lll}
\hline 3 & Beschreibung der experimentellen Technik & 12
\end{tabular}

3.1 Aufbau der experimentellen Apparatur . . . . . . . . . . . . . . . . . . . . 12

3.1 .1 Kreuzflanschkammer (Rezipient) . . . . . . . . . . . . . . . . . . . . 12

3.1 .2 Ultrahochvakuumerzeugung . . . . . . . . . . . . . . . . . . . . . . 14



3.1 .4 Auslegung der Magnetspulen. . . . . . . . . . . . . . . . . . . . . . . . 19

3.1.5 Stromquelle . . . . . . . . . . . . . . . . . . . . . . . 21

3.2 Optische Beobachtungstechnik . . . . . . . . . . . . . . . . . . . . . 22

3.2.1 Hochgeschwindigkeitskamera - Phantom . . . . . . . . . . . . . 22

3.2 .2 Übersichtskamera - Casio/EX-F1 . . . . . . . . . . . . . . . . . . . . . . . . 23

3.3 Sonstige Messtechnik . . . . . . . . . . . . . . . . . . . . . . . 23

$3.4 \quad$ Externe Möglichkeiten zur Probenuntersuchung . . . . . . . . . . . . . . . 24

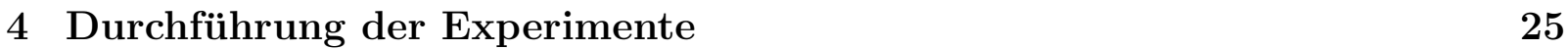

4.1 Klassifizierung des Probenmaterials . . . . . . . . . . . . . . . . . 25

4.2 Aufbau und Durchführung der Versuche . . . . . . . . . . . . . . . . . . . . 26

4.2 .1 Beobachtung der Brennfleckdynamik . . . . . . . . . . . . . . . 26

$4.2 .2 \quad$ Beobachtung der Partikelemission . . . . . . . . . . . . . . . . . . . . 28

$4.2 .3 \quad$ Beobachtung der Kathodenerosion . . . . . . . . . . . . . . . . . . 30

\begin{tabular}{|lll}
\hline 5 & Ergebnisse & 31
\end{tabular}

$5.1 \quad$ Allgemeine Beobachtungen der Versuche . . . . . . . . . . . . . . . . . . . 31

5.1 .1 Kohlenstoff $-\mathrm{C}$. . . . . . . . . . . . . . . . . . 31

5.1 .2 Wolfram $-\mathrm{W}$. . . . . . . . . . . . . . . . . . . . 35

5.1 .3 Kohlenstoff $-\mathrm{CW}$. . . . . . . . . . . . . . . . . . . . . . . . . . . . . . . . . . .

5.1 .4 Wolfram $-\mathrm{WAl}_{2} \mathrm{O}_{3} \ldots \ldots \ldots \ldots$. . . . . . . . . . . 45

5.2 Detaillierte Auswertung $\ldots \ldots \ldots \ldots$. . . . . . . . . . . . . . 46 
$5.2 .1 \quad$ Strukturebenen der Bogenfußpunkte . . . . . . . . . . . . . . . 46

$5.2 .2 \quad$ Geschwindigkeiten der Brennfleckenfront . . . . . . . . . . . . . . . 51

$5.2 .3 \quad$ Reaktion der Brennflecken auf schnelle Stromänderungen . . . . . . . 58

5.2 .4 Geschwindigkeit der Makropartikel . . . . . . . . . . . . . . . . 62

$\begin{array}{lll}6 & \text { Zusammenfassung } & 65\end{array}$

\begin{tabular}{ll}
\hline Anhang & 67
\end{tabular}

\begin{tabular}{ll}
\hline Literaturverzeichnis & 74
\end{tabular}

\begin{tabular}{ll}
\hline Danksagung & 77
\end{tabular} 


\section{Abkürzungsverzeichnis}

$\rho \ldots \ldots \ldots \ldots$ Spezifischer Widerstand, in $\frac{\Omega \cdot m m^{2}}{m}$

$B_{\text {ext }} \ldots \ldots \ldots$ magnetische Flussdichte des externen Magnetfeldes, in $\mathrm{mT}$

$B_{\text {int }} \ldots \ldots \ldots$ magnetische Flussdichte des internen Magnetfeldes, in $\mathrm{mT}$

$I_{\text {Bogen }} \ldots \ldots$. Bogenstrom, in A

$I_{\text {Spule }} \ldots \ldots \ldots$ Spulenstrom, in $\mathrm{A}$

$l_{\text {Leiter }} \ldots \ldots \ldots$ Länge des Leiter, in $\mathrm{m}$

$l_{\text {Spule }} \ldots \ldots \ldots$ Länge der Spule, in mm

$R_{B F F} \ldots \ldots$. Radius der Brennfleckfront

$t_{\text {ent }} \ldots \ldots \ldots$ Entladungsdauer, in $\mu s$

$t_{\text {interval }} \ldots \ldots$ Bildintervall, in $\mu s$

но $\ldots \ldots \ldots$ magnetische Feldkonstante $\left(4 \cdot \pi \cdot 10^{-4} \mathrm{H} / \mathrm{mm}\right)$

A $\ldots . . . \ldots$ Ampere, Si-Basiseinheit der elektrischen Stromstärke, in C/s

$\mathrm{A}_{\text {Leiter }} \ldots \ldots$ Querschnitt des Leiters, in $\mathrm{mm}^{2}$

$\operatorname{arc} \ldots \ldots \ldots$ engl. für Bogen

BFF ........ Brennfleckfront

CMOS ...... Complementary Metal Oxide Semiconductor

$\mathrm{E}_{W A} \ldots \ldots . .$. oberes Energieniveau der Austrittsarbeit, in eV

$\exp \ldots \ldots \ldots$ Belichtungszeit, in $\mu s$

fps. ........ frames per second

$\mathrm{H} \ldots \ldots \ldots$ Henry, magnetische Feldstärke, in $\mathrm{A} / \mathrm{mm}$

IPP ....... Max-Planck-Institut für Plasmaphysik

$\mathrm{m} \ldots \ldots \ldots$ Si-Längeneinheit

$\mathrm{MHz} \ldots \ldots$. Mega Hertz

$\mathrm{Pa} \ldots \ldots . .$. Pascal, abgeleitete Si-Einheit für den Druck, in $N / m^{2}$

$\mathrm{R} \ldots \ldots \ldots$ Elektrischer Widerstand, in $\Omega$

$\mathrm{r} \ldots \ldots \ldots \ldots$ Radius Leiterschleife bzw. Windungslage

UHV ....... Ultrahochvakuum

$\mathrm{V} \ldots \ldots \ldots$ abgeleitete Si-Einheit der Spannung, in J/C (Volt)

v.l.n.r ....... von links nach rechts

$\mathrm{x} \ldots \ldots \ldots \ldots$ horizontaler Abstand auf der Mittelachse der Spule, in mm 


\section{Kapitel 1}

\section{Einleitung}

Die vorliegende Arbeit untersucht bipolare Vakuumbögen auf Erste-Wand-Materialien von Fusionsreaktoren.

„Die an der ersten Wand eines Fusionsreaktors ablaufenden Wechselwirkungsprozesse sind sowohl für die Erhöhung der Standzeit der Wand als auch für die Sauberkeit des Plasmas und damit für die erreichbaren Plasmatemperaturen von größter Bedeutung. Dabei laufen gleichzeitig eine Vielzahl von Einzelteilchenwechselwirkungen und von kollektiven Prozessen ab. $\mathrm{Zu}$ den letzteren zählen Lichtbogenentladungen auf der Innenwand von Fusionsreaktoren. Diese sind von besonderer Bedeutung, weil sie als einziger Abtragsprozess in der Lage sind, Partikel direkt in das zentrale Plasma einzuschießen. Während die durch Ionenbeschuss oder thermische Verdampfung abgetragenen Atome des Wandmaterials nur durch langsame Diffusionsprozesse in zentrale Bereiche des Plasmas gelangen, emittiert eine Bogenentladung in großer Zahl mikroskopische Partikel (sog. Droplets) mit hohen Geschwindigkeiten, die unmittelbar bis in das Kernplasma vordringen bevor sie dort verdampfen.

Im Rahmen der Entwicklung von Vakuumschaltern in den 70/80ziger Jahren und der Optimierung der Vakuumbogenverdampfung in den letzten 20 Jahren wurden grundlegende Eigenschaften (der) Bogenentladungen erforscht. Für eine ganze Reihe von Materialien wurden die wichtigsten Parameter gründlich vermessen. Dies betrifft Menge, Geschwindigkeit und Komponenten (Plasma, Droplets) des abgetragen Materials, den Spot-Teilungsstrom, das Bewegungsverhalten der Spots und dessen Beeinflussung durch externe Magnetfelder und weitere Parameter. ... Das Bogenverhalten auf den typischen Materialien der ersten Wand bzw. der Limiter von Fusionsanlagen wurde bisher kaum quantitativ untersucht. Besonders schlecht ist die Datenlage bei Werkstoffen, die ihrerseits mit dünnen Schichten bedeckt sind." 1

Auf Grundlage eines Forschungsauftrages zwischen Arc Precision - Sources, Coatings and Analysis und dem Max-Planck-Institut für Plasmaphysik (IPP) soll ein Ultrahochvakuum(UHV) Pumpstand aufgebaut werden, mit dem es möglich ist, umfangreiche Untersuchungen des Brennfleckverhaltens auf Erste-Wand-Materialien von Fusionsanlagen durchzuführen. In Fusionsanlagen wurden unipolare Vakuumbögen beobachtet, während der Pumpstand bipolare Vakuumbögen zündet. Die Analogie des Brennfleckverhaltens beider ist Grundlage der Experimente. Es ergeben sich folgende Schwerpunkte.

- Untersuchung der Bewegung von Kathodenbrennflecken mit einer Hochgeschwindig- 
keitskamera

- Untersuchung des Emissionsverhalten von Makropartikel mit Rasterelektronenmikroskopie und der Hochgeschwindigkeitskamera

- Mikroskopische und fotografische Untersuchungen der Kathodenerosion

Das Max-Planck-Institut für Plasmaphysik betreibt zu Forschungszwecken verschiedene Fusionsanlagen, z.B. Wendelstein 7-X (Stellerator) in Greifswald oder ASDEX Upgrade (Tokamak) in Garchingen. Mit diesen experimentellen Anlagen soll die Gewinnung von Energie durch Verschmelzung leichter Atomkerne erforscht werden. Als Teil des Europäischen Fusionsprogramms und Mitglied der Helmholtz-Gemeinschaft Deutscher Forschungszentren erteilt das Max-Planck-Institut für Plasmaphysik dem Unternehmen Arc Precision den Forschungsauftrag: "Untersuchung der Abtragsmechanismen und der Spotbewegung von Lichtbogen-Wandentladungen auf reaktorrelevanten Materialien". [1]

Das Unternehmen Arc Precision entwickelt Plasmaquellen zur Herstellung von Beschichtungen. Die Plasmaquellen, die als Modul oder komplette Beschichtungsanlage vertrieben werden, basieren auf gefilterten Hochstrombögen, deren Voraussetzung eine stabile Vakuumbogenentladung ist. Das Bogenplasma wird mit Hilfe von Magnetspulen gefiltert, dass heißt von ungeladenen Makropartikeln getrennt. Die Filterung und der hohe Ionisationsgrad des Hochstrombogenplasmas machen die Qualität der Beschichtungen aus. Mit diesem Verfahren ist Arc Precision weltweit einer der wenigen Anbieter derartiger Plasmaquellen und verfügt über optimale Voraussetzung zur Erfüllung des Forschungsauftrages.

Die vorliegende Arbeit beinhaltet mit Kapitel 2 eine Betrachtung des derzeitigen Wissenstandes. Darin werden die Einordnung der Vakuumbogenentladung in die Gasentladungen vorgenommen, grundlegende Mechanismen des Vakuumbogen wie z.B. die Elektronenemission aus dem Kathodenmaterial erläutert und weitere typische Merkmale der Vakuumbogenentladungen besprochen. In Kapitel 3 wird der Aufbau des UHV Pumpstandes, zur Zündung und Beobachtung der Vakuumbögen und die experimentelle wie analytische Technik beschrieben. Die Versuchsanordnung und die durchgeführten Experimente sind Gegenstand von Kapitel 4. Kapitel 5 schildert die Beobachtungen der Experimente im allgemeinen und nimmt Stellung zu den drei folgenden Themen.

- Strukturebenen der Vakuumbogenbrennflecken

- Geschwindigkeitsuntersuchungen der Brennflecken bzw. der Brennfleckfront

- Die Reaktion der Kathodenbrennflecken auf schnelle Bogenstromänderung

- Geschwindigkeitsuntersuchungen der Makropartikel 


\section{Kapitel 2}

\section{Kurzer Abriss des Wissenstandes - Theorie}

\subsection{Einführung in den Vakuumbogen}

Als Plasma wird ein Gemisch aus Ladungsträgern, neutralen Teilchen und Photonen, in elektrischen, magnetischen und elektromagnetischen Feldern bezeichnet. Die Ladungsträger sind hauptsächlich negative Elektronen und positive Ionen, aber auch negative Ionen können vorkommen.

Der Transport von elektrischem Strom durch ein Plasma wird als Gasentladung bezeichnet. Es gibt eine Vielzahl von Gasentladungen mit verschiedenen Voraussetzungen und Eigenschaften. Ihnen allen gemeinsam ist der Fluss von elektrischem Strom durch ein Plasma.

Die Gasentladungen werden in drei Hauptgruppen unterteilt, Funkenentladung, Glimmentladung und Bogenentladung. Für die meisten Bogenentladungen ist der Kathodenbrennfleck charakteristisch. Bogenentladungen können bei unterschiedlichem Umgebungsdruck brennen. Oberhalb eines Druckes von ca. 100 bis 10 Pa spricht man von einem Lichtbogen, unterhalb von einem Vakuumbogen. Der Vakuumbogen ist eine Gasentladung bzw. ein Spezialfall der Bogenentladung.

Wenn die unterschiedlichen Ladungsträger im Plasma eine annähernd gleiche Temperatur bzw. Energie haben, befindet sich das Plasma im thermischen Gleichgewicht. Während sich das Plasma des Lichtbogens in thermischem Gleichgewicht befindet, gilt diese Eigenschaft für den Vakuumbogen nicht immer.

Die Ladungsträger für das Vakuumbogenplasma stammen nicht, wie bei einem Lichtbogen aus dem Gas zwischen zwei Elektroden, sondern werden, durch den Kathodenbrennfleck örtlich begrenzt, aus dem Kathodenmaterial herausgelöst. Im Kathodenbrennfleck liegt das Kathodenmaterial in vier Zuständen vor, fest, flüssig, in Dampfphase und als Plasma. „Eine weitere Bezeichnung für den Vakuumbogen ist Metalldampfentladung in einer Vakuumumgebung, denn der Bogen schafft sich sein eigenes Medium." [2] Seite ix.

Im Folgenden werden der Vakuumbogen und seine materielle Quelle, die Kathodenbrennflecken, genauer betrachtet. 


\subsection{Emission der Ladungsträger}

Eine Kombination aus thermischer Anregung und elektrischer Feldemission ermöglicht es den Elektronen, aus dem Kathodenmaterial in den Raum zwischen Kathode und Anode zu gelangen.

Innerhalb von Metallen sind Elektronen frei beweglich, sie bilden Elektronenwolken. Thermisch angeregte Elektronen befinden sich auf einem höheren Energieniveau, sodass die zum Austritt aus der Elektronenwolke benötigte und materialspezifische Austrittsarbeit $\left(\mathrm{E}_{W A}\right)$ geringer ist (Abstand zwischen Ferminiveau $(\mathrm{FN})$ und $\mathrm{E}_{W A}$ ). Wird das Metall stark genug aufgeheizt, die Elektronen thermisch angeregt, so können sie den Festkörper verlassen. Für sich genommen ist die thermische Emission von Elektronen nicht ausreichend stark genug, um die benötigten hohen Stromdichten zu erzeugen.

Nach quantenmechanischer Betrachtungsweise entspricht die Aufenthaltswahrscheinlichkeit eines Elektrons in der Elektronenwolke des Festkörpers (Potenzialtopf) einer Wellenfunktion. Außerhalb des Potentialtopfes in Abbildung 2.1 wird die Aufenthaltswahrscheinlichkeit des Elektrons durch eine exponentiell abfallende Funktion beschrieben, deren Lösung nur im unendlichen null wird. Durch Anlegen eines äußeren elektrischen Feldes bekommt die Funktion eine Lösung im Endlichen, sie entspricht der Wahrscheinlichkeit aus dem Festkörper heraus zu tunneln.

Abbildung 2.1 skizziert die thermische Anregung der Elektronen (Energieniveau 1 unangeregt und 2 angeregt), den Tunneleffekt, sowie die Kombination beider. Es ist zu erkennen, dass die Tunnelwahrscheinlichkeit mit stärkerer thermischer Anregung und höherer elektrischer Feldstärke (Anstieg des Potentials von $\left.E_{\text {Feld }}\right)$ wächst $\left(\mathrm{W}_{1}>\mathrm{W}_{2}\right)$. Je mehr Elektronen emittiert werden, desto höher werden Feldstärke und Temperatur, wodurch noch mehr Elektronen emittierte werden.
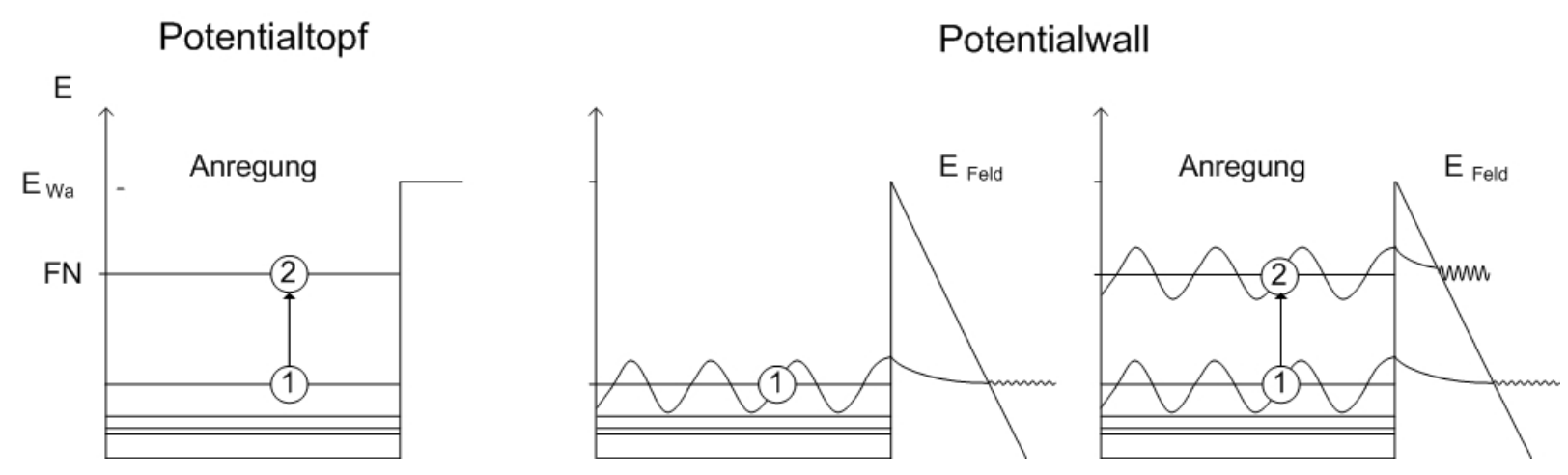

Abbildung 2.1: Thermische Anregung der elektronen im Potentialtopf, Feldemission durch Potentialwall und die Kombination beider Effekte, Thermo-Feld-Emission (Schema)

Die sehr komplexen Verhältnisse der Thermo-Feld-Emission werden in Gleichung 2.1 von E. Hantzsche [3] vereinfacht zusammengefasst. Daraus geht hervor, dass beide Effekte, thermische Anregung und Feldemission, sich gegenseitig bedingen und potenzieren. 


$$
j(T, F) \approx k\left(A T^{2}+B F^{9 / 8}\right) \exp \left[-\left(\frac{T^{2}}{C}+\frac{F^{2}}{D}\right)^{-1 / 2}\right]
$$

$j(T, F)$ Elektronenstromdichte, $T$ Temperatur, F Feldstärke, $k$ und $A$ bis $D$ sind Konstanten

Ein mögliches Modell für das Entstehen der Kathodenbrennflecken beschreibt Schrade [4]. Demnach können, aufgrund der Thermo-Feld-Emission, einige Ladungsträger in den Kathodenfallraum gelangen und vom elektrischen Feld beschleunigt werden. Die Stromdichte steigt an Mikrospitzen der Oberfläche zu Größenordnungen von $10^{12} \mathrm{~A} / \mathrm{m}^{2}$, in deren Folge die Aufheizung des Kathodenmaterials sehr viel größer ist als die Wärmeleitung bzw. Abkühlung des Kathodenmaterials. Dieses verdampft explosionsartig und wird durch Stoßprozesse mit den beschleunigten Elektronen ionisiert. Ein hoch ionisiertes Plasma breitet sich aus von der Kathode weg zur Anode hin.

\subsection{Quasineutralität, Brennspannung und Bogenstrom}

Stabile Plasmen sind quasineutral. Es gleichen sich positive und negative Ladungen auf makroskopischer Ebene aus. Auf mikroskopischer Ebene sind die Ladungen getrennt und Wechselwirken untereinander.



Abbildung 2.2: Spannungsabfall über die Bogenlänge Links. Und Bewegungsrichtung der Ladungsträger vor und nach dem Kathodenfallraum, sowie Makropartikel Rechts. Nach Martin [5]. (Skizze)

Bei Vakuumbögen von geringer Stromstärke (weniger 1kA) bleibt die Anode passiv, sie emittiert keine Ladungsträger. Dennoch ist das Bogenplasma über den Großteil der Distanz zwischen Anode und Kathode quasineutral. Da der Kathodenbrennfleck die einzige 
Ionenquelle ist, müssen die Ionen gegen das elektrische Feld des Bogenstromes beschleunigt werden. Siehe Abbildung 2.2 nach Martin [5]. Offenbar herrschen im Kathodenbrennfleck Bedingungen und Mechanismen die diese Beschleunigung zur Folge haben.

Die Bewegung der Elektronen setzt sich aus zwei Komponenten zusammen. Thermische Elektronen (geringe kinetische Energie, ca. 1 - $3 \mathrm{eV}$ ) bewegen sich ungerichtet, nur aufgrund ihrer Temperatur. Die zweite komponente ist gerichtet und resultiert aus der Beschleunigung im elektrischen Feld. Die Trennung von Elektronen und Ionen, aufgrund der zweiten Komponente führt zur ambipolaren Diffusion beider Ladungsträger in Richtung Anode.

Der Großteil der Spannung fällt über dem Kathodenfallraum ab, der nur einen Bruchteil der Länge des Bogens ausmacht (Abbildung 2.2 links). An dieser Stelle, kurz vor der Kathode sammeln sich die meisten Metallionen und bilden eine positive Raumladung. Über dem Rest der Bogenlänge fällt nur sehr wenig Spannung ab, was mit der Quasineutralität des Plasmas hinter dem Kathodenfallraum zusammenhängt.

Detaillierte Untersuchungen von Kimblin [6] haben gezeigt, dass der Ionenstrom im Plasma ca. zehn Prozent des Bogenstromes beträgt. Der Ionenstrom ist dem Bogenstrom entgegen gerichtet, weshalb er vom Elektronenstrom kompensiert werden muss. Dem entsprechend beträgt der Elektronenstrom 110\% des Bogenstromes. In Abbildung 2.3 ist der Zusammenhang von Bogenstrom und Ionenstrom dargestellt.



Abbildung 2.3: Maximaler Ionenstrom der Kathodenbrennflecken auf $\mathrm{Cd}, \mathrm{Zn}, \mathrm{Ag}, \mathrm{Cu}, \mathrm{Cr}, \mathrm{Fe}, \mathrm{Ti}$, $C$, Mo und $W$ Elektroden gegen den dc Bogenstrom von Kimblin [6].

Bei einem hohen elektrischen Stromfluss und einem geringen Spannungsabfall über den gesamten Vakuumbogen folgt ein geringer elektrischer Widerstand des Bogenplasmas. Folgendes stichprobenartiges Zahlenbeispiel verdeutlicht das. $R=\frac{U}{I}$ mit 600 Ampere Bogenstrom und 39,2 Volt Spannungsabfall, ergibt sich ein Ohmschen Widerstand von $65 \mathrm{~m} \Omega \mathrm{zu}$ 
einem Zeitpunkt von einer Millisekunde bei Entladung 18 am 05.11.2010.

\subsection{Kathodenerosion}

Der Brennfleck hinterlässt Krater, die dem Verlauf der Bogenspur entsprechen und in Abbildung 2.4 von Jakubka [7] gut sichtbar sind. Wroe untersuchte bereits 1958 Wolfram Kathoden und konnte mit Bögen von 150 A Bogenstrom und 30 ms Brenndauer hauptsächlich Krater von einem Mikrometer Kraterdurchmesser finden. [8]

Die Kathodenbrennflecken erodieren das Kathodenmaterial hauptsächlich in zwei Formen, als Plasma oder geschmolzene Makropartikel. Metalldampf hat hingegen einen sehr geringen Anteil an der erodierten Masse. 9] Die Erosionsrate ist definiert als Masse des abgelösten Metalls oder Gases in Relation zu dafür aufgewandten Ladungen. (Boxman [2] Seite 122)

Die geschmolzenen Makropartikel bestehen aus dem Kathodenmaterial und werden von den explosiven und komplexen Vorgängen im Brennfleck beschleunigt. Sie sind nahezu elektrisch neutral und haben daher keinen signifikanten Anteil am Bogenstrom, lassen sich demnach aber auch nicht von Magnetfeldern beeinflussen. Der Literatur zufolge [9], verlassen die Makropartikel die Krater in geringem Winkel (Cu: 1-20 Cd: 1-40 ) zur Oberfläche und können von Größen unter einem Mikrometer bis zu 100 Mikrometer gemessen werden.

Der größte Teil des Metalldampf wird von den beschleunigten Elektronen im Brennfleck stoßionisiert, und als Plasma emittiert. Nach Boxman [2] Seite 132 erreichen die Ionen eine Geschwindigkeit von 10-20 km/s, die kinetische Energie der Ionen liegt dem entsprechend bei $E_{k i n}=\frac{m_{i}}{2} \cdot v^{2}$.

\subsection{Brennflecktypen}

Es werden zwei Arten von Kathodenbrennflecken unterschieden, Typ 1 und Typ 2. [10] 11 Die Brennflecken vom Typ 1 bewegen sich schneller als die Brennflecken vom Typ 2. Die Krater, die Typ 1 hinterlässt sind klein und diskontinuierlich angeordnet und verursachen weniger Erosion. Im Gegensatz zu den großen, sich überlappenden Kratern der Brennflecken vom Typ 2.

Typ 1 tritt auf kontaminierten Oberflächen auf, dass heißt auf dünnen dielektrischen Schichten von einigen Nanometern Schichtdicke. Das können sowohl natürliche Oxidschichten als auch Wasser oder eventuelle Bearbeitungsrückstände sein. Typ 2 tritt auf sauberen, dass heißt rein metallischen Oberflächen auf. Bei den ersten Brennflecken auf einer Kathode ist die Erosion des Metalls niedrig weil die Oberfläche noch kontaminiert ist. Mit steigender Anzahl aufeinander folgender Entladungen ändert der Brennfleck seiner Erscheinung. Dies entspricht der Änderung von Typ 1 hin zu Typ 2. Die Brennflecken formieren die Kathodenoberfläche. 
Abbildung 2.4 zeigt Bogenspuren die von beiden Brennflecktypen verursacht wurden. Der Übergang von Typ 1 zu Typ 2 ist mit schwarzen Pfeilen markiert.



Abbildung 2.4: Foto von Kathodenerosion durch beide Brennflecktypen, I und II jeweils in weiß beschriftet; Übergang mit schwarzen Pfeilen gekennzeichnet; von Jakubka [7]

\subsection{Brennfleckbewegung}

Der Anschein der Brennfleckbewegung entsteht durch die Auslöschung und Neubildung der Brennflecken. Die Bewegung ist also diskontinuierlich (Boxman [2] Seite 93 folgend). Die Zeit zwischen zwei aufeinander folgenden Brennflecken, deren Lebensdauer und die Schrittweite der Brennflecken variieren bei verschiedenen Parametern, wie z.B. dem Material oder einem externen Magnetfeld. Gemittelt werde sie als Geschwindigkeit der Brennfleckfront für den jeweiligen Parameter angegeben.

Die Richtung der Brennfleckbewegung ist durch zwei Komponenten bestimmt. Eine chaotische Komponente und eine gerichtete Komponente. Eine mögliche Erklärung der chaotischen Komponente ist die Brennfleckzündung an Mikrospitzen und damit die Oberflächen- 
beschaffenheit der Kathode. Ein einzelner Brennfleck bewegt sich ausschließlich chaotisch (siehe 1a in Abbildung 2.5).

Die gerichtete Komponente der Brennfleckbewegung resultiert aus Magnetfeldern, diese setzten sich aus internen (eigenen) und externen Magnetfeldern zusammen (Boxman [2] Seite 100).(siehe 1b bzw. 1c mit stärkerem Feld, eben da). Die gerichtete Komponente der Bewegung wird mit höherer magnetischer Flussdichte stärker, wobei die chaotische Komponente nie ganz verschwindet. [12]

$1 \mathrm{a}$

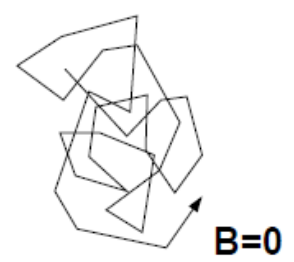

$1 \mathrm{~b}$

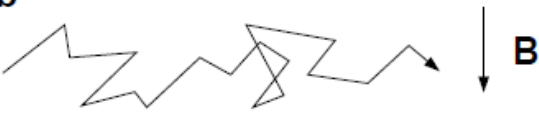

1c

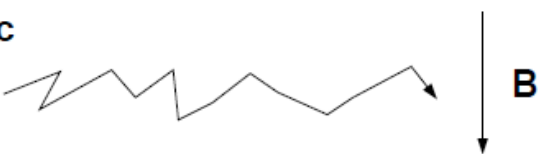

Abbildung 2.5: Einzelbrennfleckbewegung mit schwachem, starken und ohne externem Magnetfeld (1) (Shema) von Kleberg [13].

Bei einer für das Kathodenmaterial spezifischen Stromstärke, teilen sich die Brennflecken auf und tragen jeweils einen Teil des Stroms weiter. (Abbildung 2.6) Der Abbildung ist zu entnehmen, dass es für die Brennfleckteilung einen konkreten Schwellenwert gibt. Bei Kupfer zum Beispiel tritt die Teilung zwischen 60 und 150 Ampere ein. [14

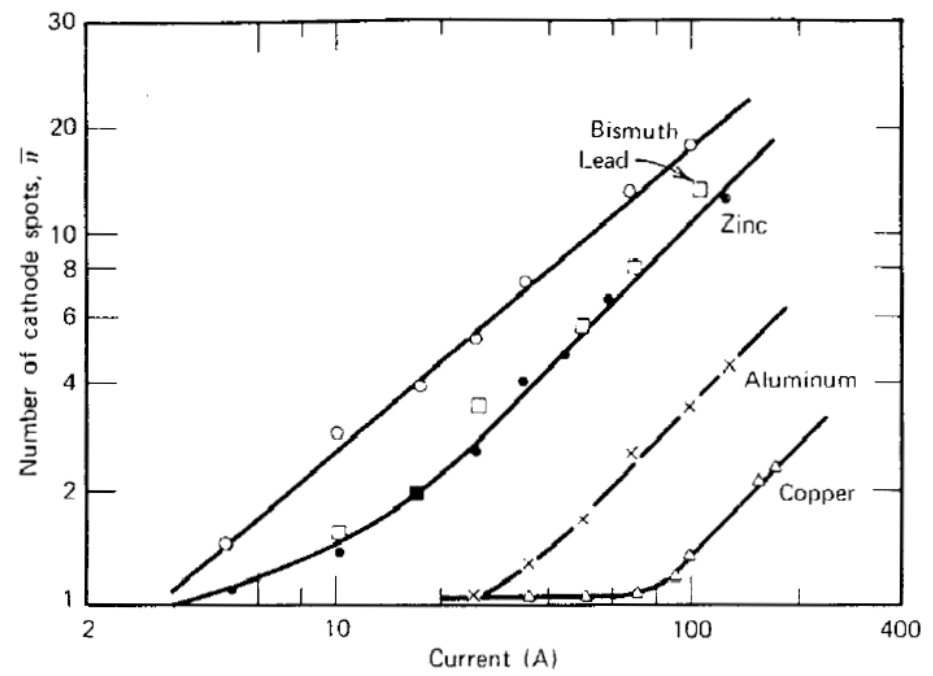

Abbildung 2.6: Anzahl der Brennflecken als Funktion des Bogenstroms für Bi, $\mathrm{Pb}, \mathrm{Zn}, \mathrm{Al}$ und $\mathrm{Cu}$ von Djakov und Holmes [14]

Mehrere Brennflecken wechselwirken miteinander aufgrund ihrer eigenen (internen) magnetischen Felder und bekommen eine gerichtete Komponente [15]. Sie werden als gerade stromdurchflossene Leiter betrachtet. Mehrere Brennflecken stoßen sich ohne ein äußeres Magnetfeld gegenseitig ab (Abbildung 2.7 2a). Nach dem Model des stromdurchflossenen Leiters müssten sich mehrere Brennflecken eigentlich anziehen, aber aufgrund der retrograden Brennfleckbewegung (siehe weiter unten) „bildet sich ein expandierender Ring“ (Boxman [2] Seite 100). Bei mehreren Brennflecken und einem externen Magnetfeld addieren 
sich beide Felder (internes und externes Magnetfeld). Es stellt sich eine Hufeisen ähnliche Brennfleckfront ein, die sich aus beiden Richtungskomponenten ergibt.

2a

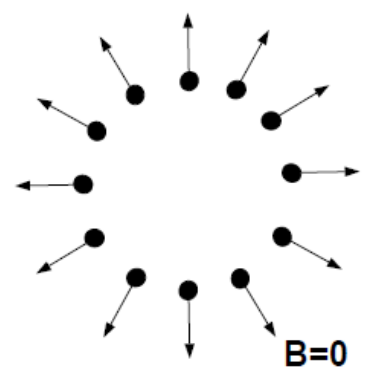

$2 b$

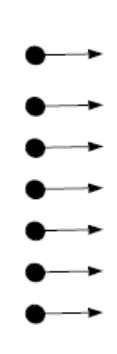

B

Abbildung 2.7: Kollektive Brennfleckbewegung mit und ohne externem Magnetfeld (2) (Shema) von Kleberg [13].

Perskie et al. [12] konnten für die Geschwindigkeit („Displacements rate“) der Brennfleckfront (,grouped cathode spots“) auf Kupfer, Cadmium und Molybdän eine lineare Abhängigkeit von einem externen transversalen Magnetfeld sowie eine Sättigung der Abhängigkeit bei Flussdichten über $400 \mathrm{mT}$, feststellen.

Von grundlegender Bedeutung für die Bewegung von Brennflecken im eigenen wie im externen Magnetfeld sind zwei Phänomene, die retrograde Bewegung und die Robsondrift, [2] Seite 93 ff. Der Brennfleck bewegt sich bei einem angelegten Magnetfeld entgegengesetzt zur Lorentzkraft, dieses Phänomen wird retrograde Bewegung genannt. Es ist ein Hauptmerkmal durch den sich der Vakuumbogenbrennfleck vom Bogenbrennfleck in Atmosphärendruck unterscheidet.

Die Abweichung von der retrograden Bewegung wird Robsondrift genannt. Dieses Phänomen tritt auf, wenn die Magnetfeldlinien nicht parallel zur Oberfläche verlaufen und führt zu einer drift ähnlichen Richtungsänderung der Brennflecken. [16

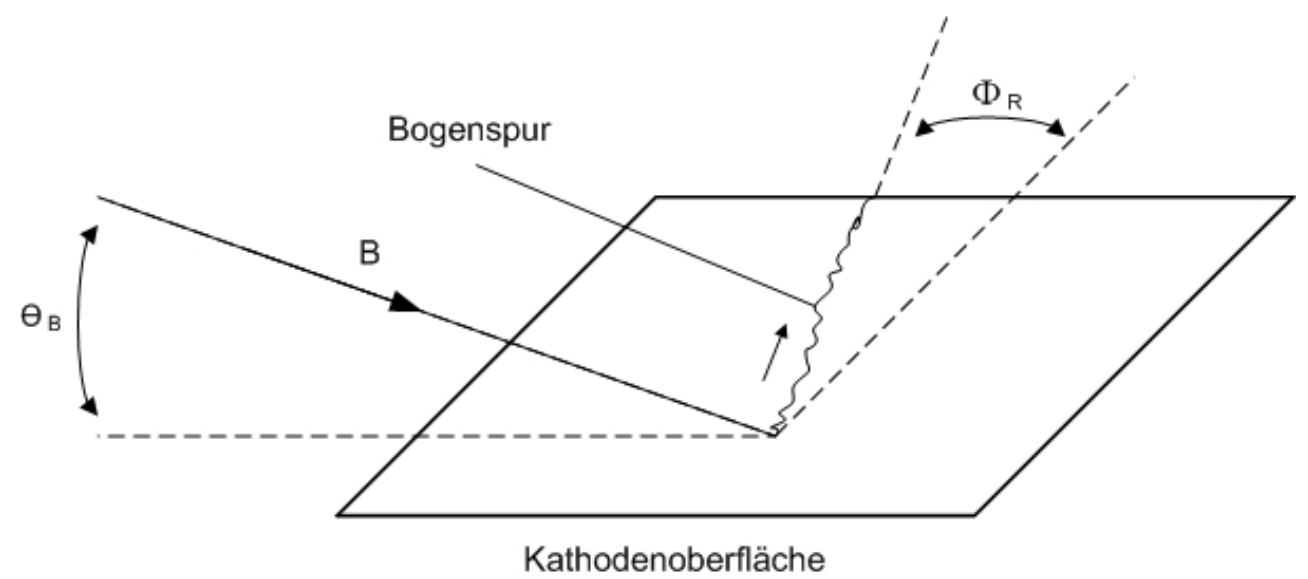

Abbildung 2.8: Robsondrift von Boxman [2] Seite 99, Definition des Robsonwinkels $\phi_{R}$, wenn das Magnetfeld im Winkel $\Theta_{B}$ auf die Kathodenoberfläche trifft (Skizze) 


\section{Kapitel 3}

\section{Beschreibung der experimentellen Technik}

Das folgende Kapitel handelt vom Aufbau der experimentellen Apparatur, der Initiierung von Vakuumbögen, der Konstruktion der Magnetspulen und der Beschreibung der weiteren experimentellen Technik, mit deren Hilfe die Bogenuntersuchungen durchgeführt werden sollen.

\subsection{Aufbau der experimentellen Apparatur}

\subsubsection{Kreuzflanschkammer (Rezipient)}

Die Bögen werden bei niedrigem Umgebungsdruck gezündet. Sie sind dem entsprechend im Rezipienten isoliert von der Umgebung und müssen dennoch mit Strom (0,5 kA bis 3 $\mathrm{kA}$ ) versorgt und auf unterschiedlichem Wege beobachtet werden. Eine Kreuzflanschkammer wurde entwickelt, die diesen Ansprüchen gerecht wird. Sie wurde von Prof. Dipl. Ing. K. Siemroth, Mitarbeiterin der Firma Arc Precision und Professorin an der TH-Wildau, konstruiert. Ein Teil der Konstruktionszeichnung ist in Abbildung 3.1 zu sehen.

Die Kammer ist aus Edelstahl gefertigt und somit nicht magnetisch. Sie wird mit Conflat Flanschen DN100 und Kupferdichtringen abgedichtete, um eine möglichst geringe Leckagerate zu erhalten. Der untere Flansch wird direkt an die eine Turbomolekularpumpe angeschlossen.

Weiterer elementarer Bestandteil der Kammer sind Stromdurchführungen an zwei der vier CF100 Flanschenenden. Sie ermöglichen die Bogenexperimente im Inneren der Kammer. Hier können die zu untersuchenden Kathoden (Erste-Wand-Materialien) sowie Siliziumwafer zum Auffangen von Makropartikeln befestigt und gegebenenfalls mit Strom versorgt werden. An den Stromdurchführungen wird ein Oszilloskop angeschlossen.

Ein Sichtglas in einem Flansch der Kammer ermöglicht die optischen Beobachtung der Experimente mit Hilfe von zwei Kameras.

Aufgrund der kompakten Form der Kammer kann von beiden Seiten eine Magnetspule angebracht werden, die ein möglichst homogenes Magnetfeld von hoher Stärke im Inneren der Kammer erzeugt. 


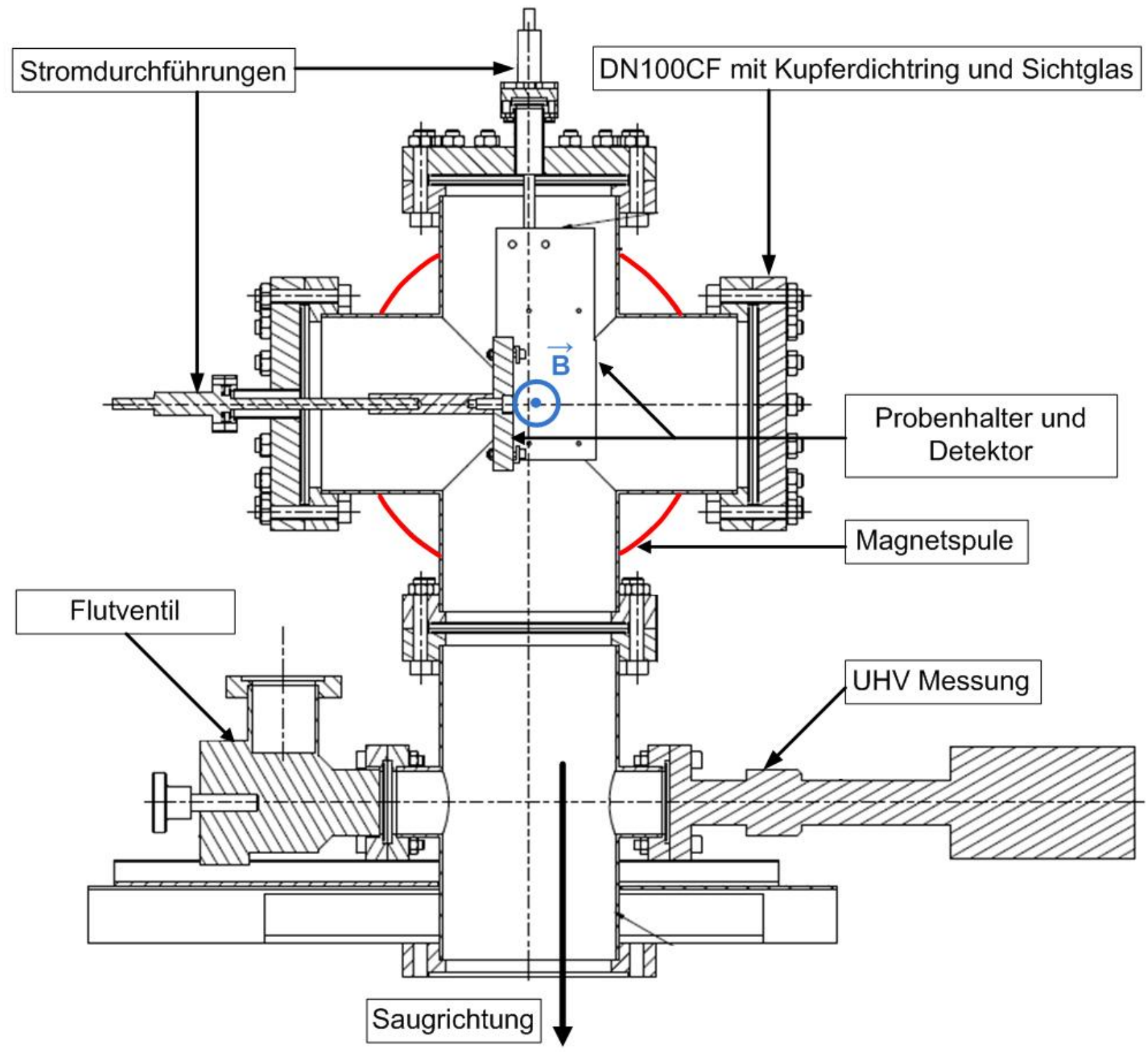

Abbildung 3.1: Querschnitt der Kreuzflanschkammer, mit Stromdurchführungen, DN100CF Flansche, Sichtglas, Probenhalter und Detektor, Magnetspule (rot) und UHV Messung (Konstruktionszeichnung) 


\subsubsection{Ultrahochvakuumerzeugung}

Um die Bögen zu zünden, wird in der Kreuzflanschkammer UHV erzeugt. Der Bogen zündet bereits bei Drücken zwischen $100 \mathrm{~Pa}$ und $10 \mathrm{~Pa}$, es soll jedoch ein Druck erzeugt werden, der auch in Fusionsanlagen vorkommt und somit dem Hochvakuum bzw. dem Ultrahochvakuum entspricht. Dieses Vorgehen erleichtert nicht nur das Vergleichen der Versuchssergebnisse mit den Beobachtungen aus den Fusionsanlagen, sonder garantiert auch die Vergleichbarkeit mit den aus der Literatur bereits vorhandenen Ergebnissen.

Das UHV wird mechanisch durch eine Vorvakuumpumpe mit nachgeschalteter Turbomolekularpumpe erzeugt und beginnt bei einen Druck von $10^{-5} \mathrm{~Pa}$. Das zusätzliche Ausheizen des Rezipienten beschleunigt die Erzeugung des UHVs erheblich.

Die Turbopumpe (Turbovac 360 mit Stromversorgungs- und Steuerungseinheit Turbotronik NT 150/36; Leybold-Heraeus) setzt abluftseitig ein Vorvakuum (ca. 10 Pa) voraus. Das Vorvakuum wird mit einer Drehschieberpumpe (Duo 5 MC; Pfeifer Vacuum) erzeugt.

Aus der mit Öl geschmierten Drehschieberpumpe wird feiner Ölnebel, auch gegen die Saugrichtung der Pumpe, abgegeben und kann durch die Turbopumpe bis in den Rezipienten vordringen, und dort zu Verunreinigungen führen. Deshalb ist zwischen den beiden Pumpen eine Zeolith- bzw. Adsorbtionsfalle der Firma Vacom eingebaut. Sie kann den Ölnebel adsorbieren. Der Einbau einer Zeolithfalle ist nicht nötig, wenn eine Membranpumpe zur Erzeugung des Vorvakuums eingesetzt wird.

Des weiteren sind DN25KF Absperrventile und Pirani Druckmessungen mit einem Messbereich von $10^{5} \mathrm{~Pa}$ bis $10^{-1} \mathrm{~Pa}$ an der Vorvakuumstrecke (Abbildung 3.2 angebracht, um die Belüftung der Zeolithfalle zu verhindern und das Vorvakuum zu kontrollieren.(Thermovac TR205/TM210; Leybold-Heraeus und PIA 100; Ilmvac)

Auf der Vorvakuumseite sind alle Verbindungen als Klammerflansch (DN25KF und DN16KF) mit Gummidichtungen ausgeführt, während auf der Ultrahochvakuumseite die Verbindungen als Conflat (CF40; CF100) mit Kupferdichtring realisiert werden. Metallische Dichtungen haben eine geringere Leckagerate, sind also unabdingbar für ein UHV, können aber in den meisten Fällen nur einmal benutzt werden. Da die Ultrahochvakuumseite später ausgeheizt werden soll und wegen der hohen Leckagerate dürfen auf der Ultrahochvakuumseite keine Gummidichtungen benutzt werden.

Bei geöffnetem Rezipienten wird Wasser aus der Umgebungsluft von der Innenwand des Rezipienten adsorbiert. Bei geschlossenem Rezipienten kann es nur mit sehr geringer Wahrscheinlichkeit von der Turbopumpe abgesaugt werden. Dem entsprechend sinkt der Druck im Rezipienten nach einer gewissen Pumpdauer nur noch sehr langsam, denn der Partialdruck von Wasser trägt zur Erhöhung des Druckes im Rezipienten bei. Das Wasser kann durch Zuführen von thermischer Energie von der Innenwand der Kreuzflanschkammer desorbiert werden. Die daraus resultierende Vorgehensweise wird ausheizen genannt. Je sorgfältiger das Ausheizen durchgeführt wird desto mehr Wasser kann abgesaugt werden und niedrigere Drücke sind erreichbar, bis kein Wasser mehr im Rezipienten vorhanden ist. Hierbei sind Kaltstellen zu vermeiden, da der Druck im Rezipienten nur so weit sinkt wie an der kältesten Stelle.

Zum Ausheizen wurde eine speziell für die Kreuzflanschkammer konzipierte Aluminium- 

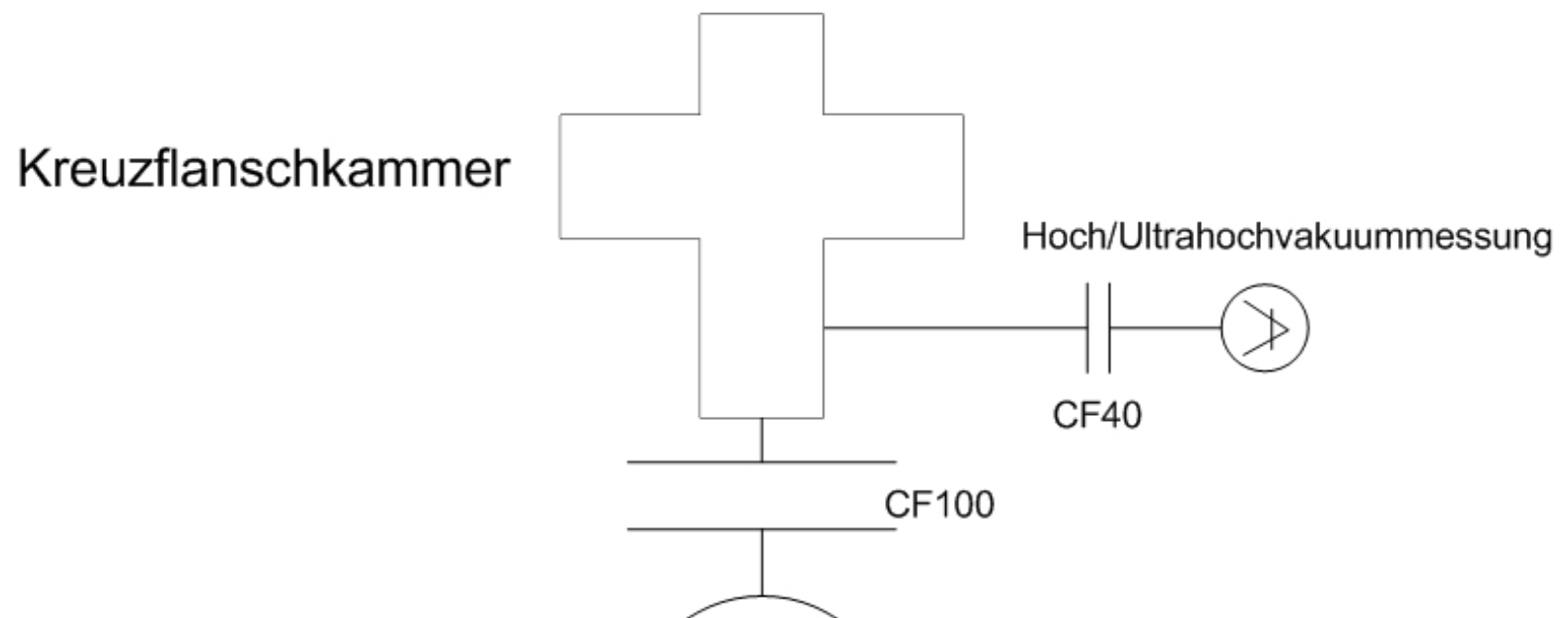

Belüftungsventil

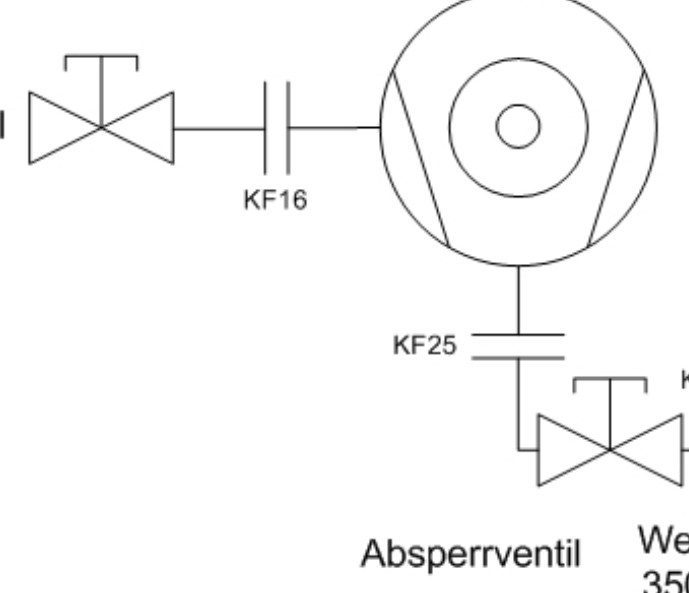

Turbopumpe

Wellenschlauch $400 \mathrm{~mm} \times \mathrm{KF} 25$

1

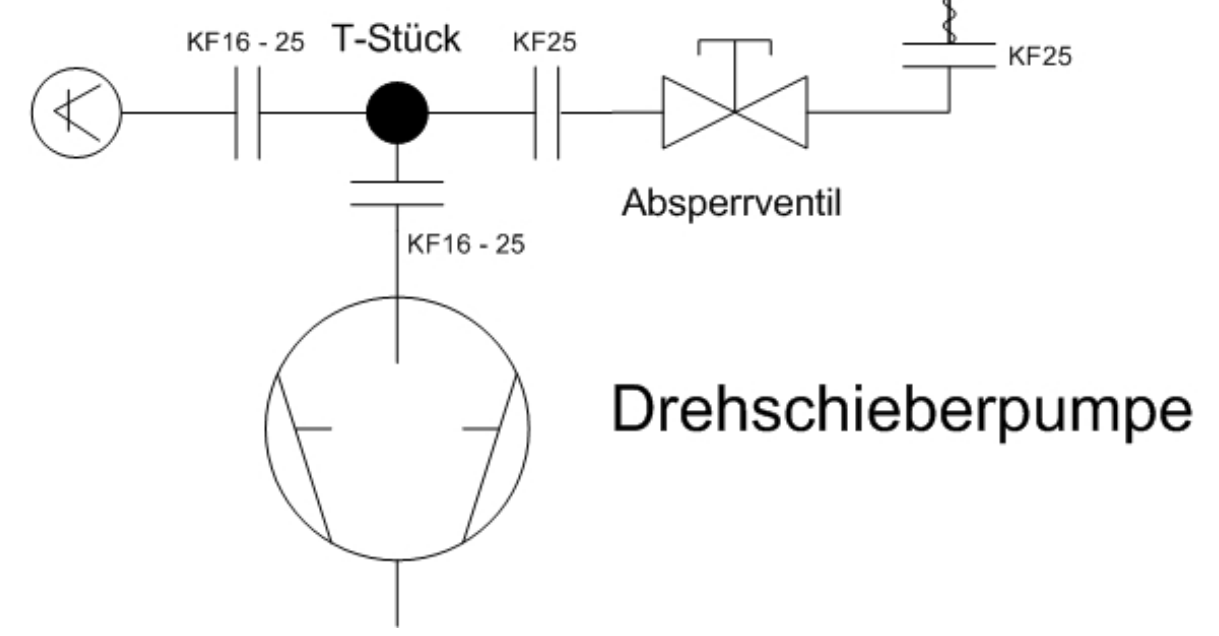

Abbildung 3.2: Plan der Vakuumstrecke, Drehschieberpumpe, Zeolithfalle, Turbomolekularpumpe und Kreuzflanschkammer 
haube entwickelt. An ihrer Innenwand sind sechs Halogenstablampen mit jeweils $500 \mathrm{~W}$ elektrischer Leistung angebracht. Üblicherweise werden zum Ausheizen Manschetten mit elektrischen Widerstandsheizungen an der gesamten Anlage angebracht und die Anlage mit wärmebeständigen und isolierenden Stoffen eingehüllt. Aufgrund der Kompaktheit der Kreuzflanschkammer kann auf diesen Aufwand jedoch verzichtet werden. Statt dessen wird die Haube über den Rezipienten gestellt.

Die Erhitzung des Pumpstandes soll möglichst homogen stattfinden, da sonst thermische Spannungen zu Schäden am Pumpstand führen können. Die Lampen sind entsprechend einer möglichst homogenen Wärmeverteilung angeordnet, zusätzlich wird die Strahlung aufgrund des hohen Reflexionsgrades von Aluminium unter der Haube immer wieder reflektiert und somit verteilt. Darüber hinaus ist auf ein langsames Ansteigen der Temperatur zu achten.

Es wurde bei Temperaturen von $150{ }^{\circ} \mathrm{C}$ bis $250{ }^{\circ} \mathrm{C}$ ausgeheizt, wobei die Höchsttemperaturen der verwendeten Bauteile, wie z.B. des Sichtglases, der Stromdurchführung, oder des Flanschs der Turbopumpe zu beachten ist. Diese sensiblen Bereiche wurden mit Aluminiumfolie umwickelt und somit vor direkter Einstrahlung geschützt, was in einem geringeren Anstieg der Graphen in Abbildung 3.3 zu erkennen ist.



Abbildung 3.3: Temperaturverlauf an verschiedenen Komponenten des Pumpstandes während des Ausheizvorgangs (Stromdurchführungen, Reduzierkreuzflansch (nahe Ionivac) und Fenster

Die Heizkurven vom 17.11.10 zeigt den Temperaturverlauf während des Ausheizvorgangs an vier zu überwachenden Stellen des Pumpstandes. Nachdem der Rezipient abkühlen konnte wurde am 18.11.10 ein Druck von $1,5 \cdot 10^{-7}$ Pa erreicht. Der Ausgangsdruck am 17.11.10 
betrug $1,7 \cdot 10^{-6} \mathrm{~Pa}$, also eine Größenordnung mehr. Der erreichte Druck entspricht dem vorderen UHV. Der Druck des Vakuums wurde mit einem Ionisationsvakuummeter mit Glühkathode nach Bayard-Alpert (Ionivac IM220, Messbereich ca. $10^{-1} \mathrm{~Pa}$ bis $10^{-10} \mathrm{~Pa}$ ) gemessen.

Um zu verhindern, dass die Kreuzflanschkammer nach jedem Umbau der Versuchsanordnung von neuem ausgeheizt werden muss, wird eine Stickstoffleitung an das Belüftungsventil des Pumpstandes angebracht, denn Stickstoff wird weniger adsorbiert als Wasser und kann schnell wieder abgepumpt werden.

Eine Havarie der Turbopumpe, ausgelöst durch Lagerverschleiß der gebrauchten Turbopumpe, machte den Austausch der Turbopumpe nötig. Die Kreuzflanschkammer wurde auf einen vollautomatischen Pumpstand (TSU 520D Pfeifer Vakuum) montiert. Letzterer umfasst eine Turbopumpe, eine Membranpumpe (MDAT Vacuubrand), sowie die Antriebselektronik. (TCP 380; Pfeifer Vacuum)

\subsubsection{Bogeninitiierung}

Die Existenz eines Bogens, der einen hohen Strom bei einem niedrigen Spannungsabfall leiten kann, hat zwei Voraussetzungen. Ein aktiver Kathodenbrennfleck muss vorhanden sein und ein durchgehendes Plasma muss die Kathode mit der Anode verbinden.

Um diese Voraussetzungen zu schaffen gibt es mehrere Möglichkeiten. Die mechanische Trennung von zwei zuvor kontaktierten Elektroden besitzt den Vorteil, dass der Raum zwischen Anode und Kathode sofort mit Plasma gefüllt ist. Im Gegensatz dazu stehen die Möglichkeiten der Zündung mittels Laserstrahlung oder Hochspannungsdurchschlag. Hierbei wird ein primärer Brennfleck auf der Kathode initiiert, dessen Plasma erst die Anode erreichen muss um einen stabilen Bogen zu erzeugen.

Da die erforderliche Stromquelle (Kapitel 3.1.5) bereits vorhanden ist, wurde für die Bogenuntersuchungen ein Aufbau mit einer Hilfs- bzw. Zündelektrode gewählt. Die Anordnung ist in Abbildung 3.4 skizziert und fotografiert. Um den aus Keramik gefertigten und mit

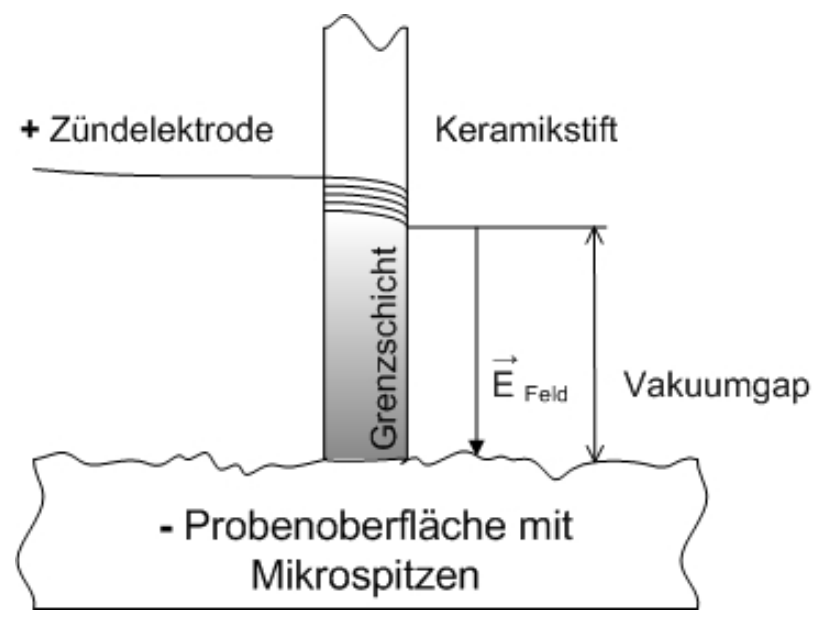

Abbildung 3.4: Querschnitt durch Kathode mit Zündelektrode, keramischer Zündstifft, Vakuumgap und Feldlinien. (Skizze) 
Kohlenstoff beschichteten Zündstift ist ein Wolframdraht gewickelt, der an einer anderen Stromdurchführung angebracht wurde und als Zünddraht (Zündanode) bezeichnet wird. Der Zünddraht leitet einen Hochspannungsimpuls zum Zündstifft. Der Hochspannungsimpuls erzeugt zunächst eine Gleitentladung. Diese verläuft längs zu den Feldlinien auf der Grenzschicht zwischen dem isolierenden Keramikstift und der Kohlenstoffschicht. Die Gleitentladung verringert die Durchschlagsfestigkeit der Anordnung gegenüber einem Vakuumgap gleicher Distanz deutlich.

Auf die Gleitentladung folgt der Durchschlag, er tritt an kleinen Spitzen an der Oberfläche auf. Dort sind die Feldstärken sehr hoch, so dass Feldemission stattfindet. An diesen Spitzen steigt die Stromdichte, was zu elektrischer Heizung führt und wiederum den Effekt der Feldemission verstärkt. [17] Unter der hohen Stromdichte wird das Kathodenmaterial explosionsartig zerstäubt und ionisiert, ein Plasma von hohem Druck wird gebildet. Der primäre Brennfleck bildet sich aus und speist das Plasma, welches sich in den Halbraum zur zweiten Anode ausbreitet.

Der Probenhalter besteht aus Kupfer und ist an einer der Stromdurchführungen angebracht. Er leitet den Entladungsstrom in die Probe. Verbindet das Plasma Kathode und Anode, in diesem Fall das Gehäuse, kann es den Entladungsstrom leiten.



Abbildung 3.5: Osszillogramm des Zündvorganges einer Vakuumbogenentladung

In Abbildung 3.5 sind der Hochspannungsimpuls (schnelles oszillieren bei 0 Sekunden) und der Zündstrom (blau) zu erkennen. Ebenso sind der Bogenstrom (grün) und die Brennspannung (rot) zu erkennen. Nach 3,6 $\mu$ s bleibt die Brennspannung von ca. 50 Volt bestehen. $\mathrm{Zu}$ diesem Zeitpunkt hat das Plasma die Anode erreicht und der Bogenstrom kann fließen (Anstieg der grünen Kurve). Der stabile Bogen brennt unselbstständig. 


\subsubsection{Auslegung der Magnetspulen}

Um die Verhaltensweisen des Vakuumbogens bzw. der Kathodenbrennfleckbewegung im externen Magnetfeld zu untersuchen wurden zwei Magnetspulen gewickelt. Die Bogenexperimente werden zwischen ihnen durchgeführt, hier soll das Magnetfeld möglichst homogen sein.

Bei der Auslegung der Spulen muss beachtet werden, dass aufgrund des erhöhten Umfangs der Windungen, bei jeder zusätzlichen Lage nebeneinander liegender Windungen, die Stärkung des Feldes sinkt und der ohmsche Widerstand steigt.

Die horizontalen Abmaße der Spulenkörper wurden mit $50 \mathrm{~mm}$ konstruktiv vorgegeben, dass heißt es konnten bei $6 \mathrm{~mm}$ Leiterdurchmesser bzw. $7 \mathrm{~mm}$ Durchmesser von Kupferrohr und Schrumpfschlauch sieben Windungen nebeneinander aufgewickelt werden. Drei Alternativen wurden berechnet, um für die oben genannte Problematik eine Lösung zu finden. Danach kann die Spule aus sieben, acht oder neun übereinander liegenden Windungslagen aufgebaut werden. Jede zusätzliche Lage erhöht dabei den Windungsradius um $7 \mathrm{~mm}$ Leiterdurchmesser. Die Länge $l_{\text {Leiter }}$ der zu verwendenden Kupferrohre entspricht für beide Spulenkörper $27 \mathrm{~m}, 37 \mathrm{~m}$ und $44 \mathrm{~m}$. Den drei Varianten entsprach bei einem Rohrquerschnitt $\mathrm{A}_{\text {Leiter }}$ von 15,7 $\mathrm{mm}^{2}$ und dem spezifischen Widerstand von Kupfer mit $\rho=1,78 * 10^{-2} \frac{\Omega m^{2}}{m}$ nach Gleichung $R=\rho * \frac{l_{\text {Leiter }}}{A_{\text {Leiter }}}$, jeweils ein ohmscher Widerstand von ca. $30 \mathrm{~m} \Omega, 43 \mathrm{~m} \Omega$ und $50 \mathrm{~m} \Omega$. Des weiteren müssen $7 \mathrm{~m}$ Kabel für die Verbindung zur Stromquelle einbezogen werden, was jeweils zu einem zusätzlichen Widerstand von 5 $\Omega$ führt.

In Abhängigkeit des Spulenstromes wurde unter Zuhilfenahme der Formel 3.1 von [18 die magnetische Feldstärke bzw. die magnetische Flussdichte im Mittelpunkt zwischen den beiden Spulen für alle drei Varianten bestimmt. Wobei $\mathrm{N}$ die Windungszahl, $l$ die Länge eines der beiden Spulenkörper, $r$ der Radius der entsprechenden Windungslagen und $x$ der horizontale Abstand auf der X-Achse von der Spulenkörpermitte ist. Das Ergebnis ergibt für $I_{\text {Spule }}=800 \mathrm{~A}, x=83 \mathrm{~mm}, l_{\text {Spule }}=49 \mathrm{~mm}$ und die entsprechenden Radien der Windungslagen eine magnetische Feldstärke von 81, 95, $101 \mathrm{~A} / \mathrm{mm}$ für sieben, acht und neun Windungslagen durch eine der beiden Spulen. Das Ergebnis muss mit $\mu o=4 \cdot \pi \cdot 10^{-4} \mathrm{H} / \mathrm{mm}$ multipliziert und verdoppelt werden, um die magnetische Flussdichte zwischen den beiden Spulenkörpern nach Formel 3.1 zu erhalten (180, 212, $244 \mathrm{mT})$. Die Ergebnisse für 7 Windungslagen sind in Tabelle 3.1 (letzte Spalte) dargestellt.

$$
H(x)=\left(\frac{I * N}{2 * l}\right) *\left[\frac{\frac{l}{2}-x}{\sqrt{\left(\frac{l}{2}-x\right)^{2}+r^{2}}}+\frac{\frac{l}{2}+x}{\sqrt{\left(\frac{l}{2}+x\right)^{2}+r^{2}}}\right]
$$

Tatsächlich aufgewickelt wurden siebeneinhalb Lagen. Mit einem Teslameter (Kapitel 3.3) wurde die magnetische Flussdichte auf dem Mittelpunkt der Spulenkörperachse vermessen. Die Messung erfolgte längs der Magnetfeldlinien. Nach dem Anfertigen der Magentspule konnte die maximale Flussdichte bei einer Einstellung von 800 A Spulenstrom gemessen werden, sie betrug $144 \mathrm{mT}$. Weitere Windungen, das heißt Lage acht und neun hätten den maximal erreichbaren Strom gedrosselt und das Feld geschwächt. 


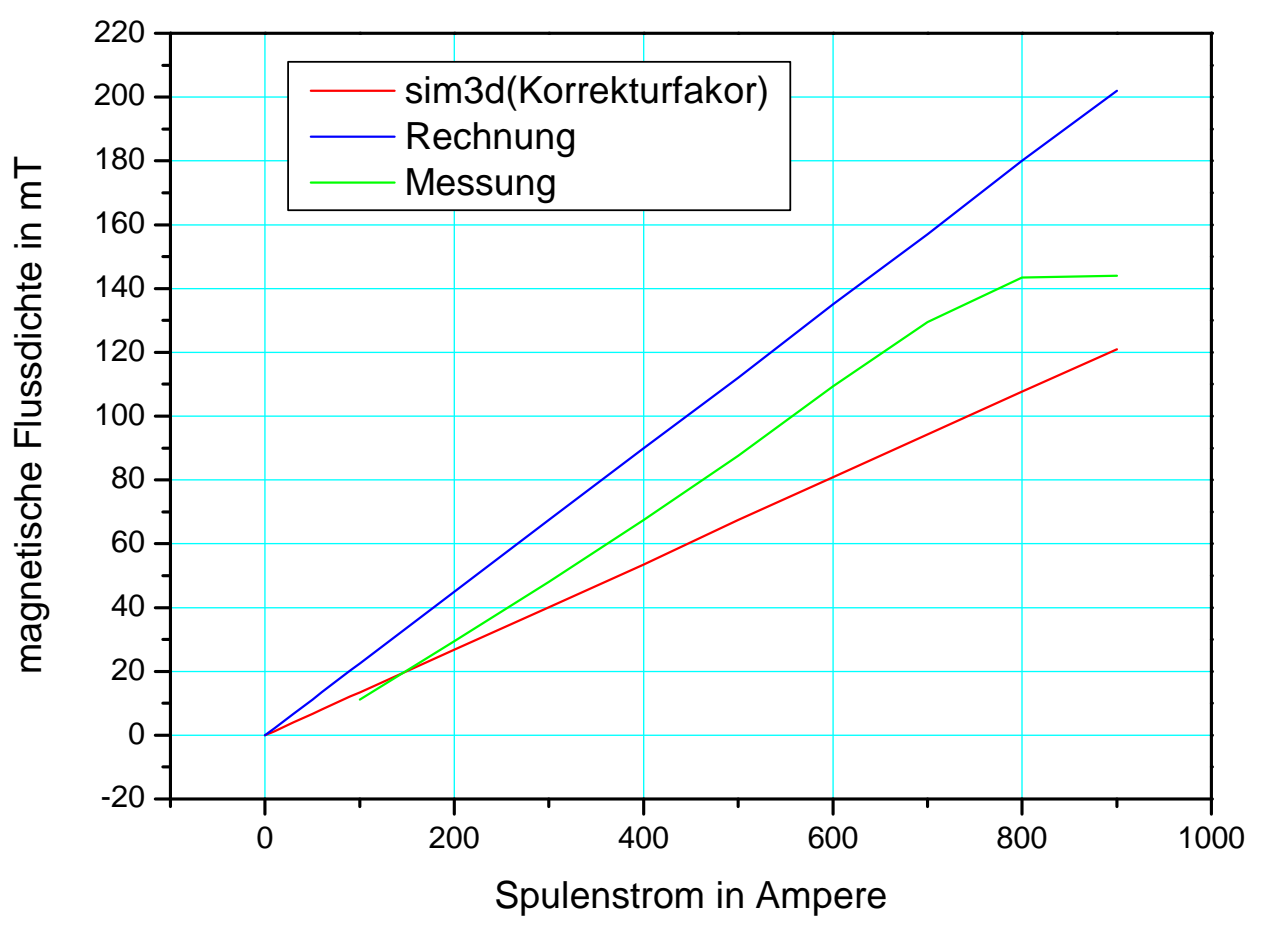

Abbildung 3.6: Magnetische Flussdichte über dem Spulenstrom, berechnet nach Formel 3.1, simuliert (mit Korrekturfaktor) und gemessen.

Tabelle 3.1: Spulenstrom (Einstellung) sowie Spannungsabfall und die resultierende Flussdichte zwischen den beiden Spulen, gemessen (grün), simuliert (rot)(mit Korrekturfaktor) und berechnet nach Formel 3.1 (blau)

\begin{tabular}{cccc}
\hline $\begin{array}{c}\text { Spulenstrom } \\
\text { in A } \\
\text { (Einstellung) }\end{array}$ & $\begin{array}{c}\text { Flussdichte } \\
\text { in mT } \pm 2 \% \\
\text { (Messung) }\end{array}$ & $\begin{array}{c}\text { Simulation } \\
\text { (mit } \\
\text { Korrekturfaktor) }\end{array}$ & $\begin{array}{c}\text { Rechnung } \\
\text { nach }\end{array}$ \\
\hline 0 & 0 & 0 & 0 \\
100 & 11,1 & 13,4 & 22,5 \\
200 & 29,5 & 26,7 & 45 \\
300 & 48 & 40,1 & 67,5 \\
400 & 67,5 & 53,5 & 90,5 \\
500 & 87,6 & 67,5 & 112 \\
600 & 109,3 & 80,9 & 135 \\
700 & 129,5 & 94,3 & 157 \\
800 & 143,4 & 107,6 & 180 \\
900 & 144 & 121,0 & 202 \\
\hline
\end{tabular}

Abbildung 3.7 zeigt die Ergebnisse einer FEM Simulation, die von Dipl. Ing. (FH) J. Richter, Mitarbeiter der Firma Arc Precision, mit der Software Femm 4.2, erstellt wurde. Die Simulation wurde bei 500 Ampere Spulenstrom berechnet und gilt für eine zweidimensionalen Ebene auf der Spulenkörpermittelachse. Um die Ergebnisse auf den Raum zu 
übertragen müssen sie mit $0,5 \pi$ multipliziert werden. Zusätzlich ist zwischen den beiden Spulen der Probenhalter angedeutet. Am Verlauf der Feldlinien ist zu erkennen, dass das Magnetfeld an dieser Stelle nahezu homogen ist.

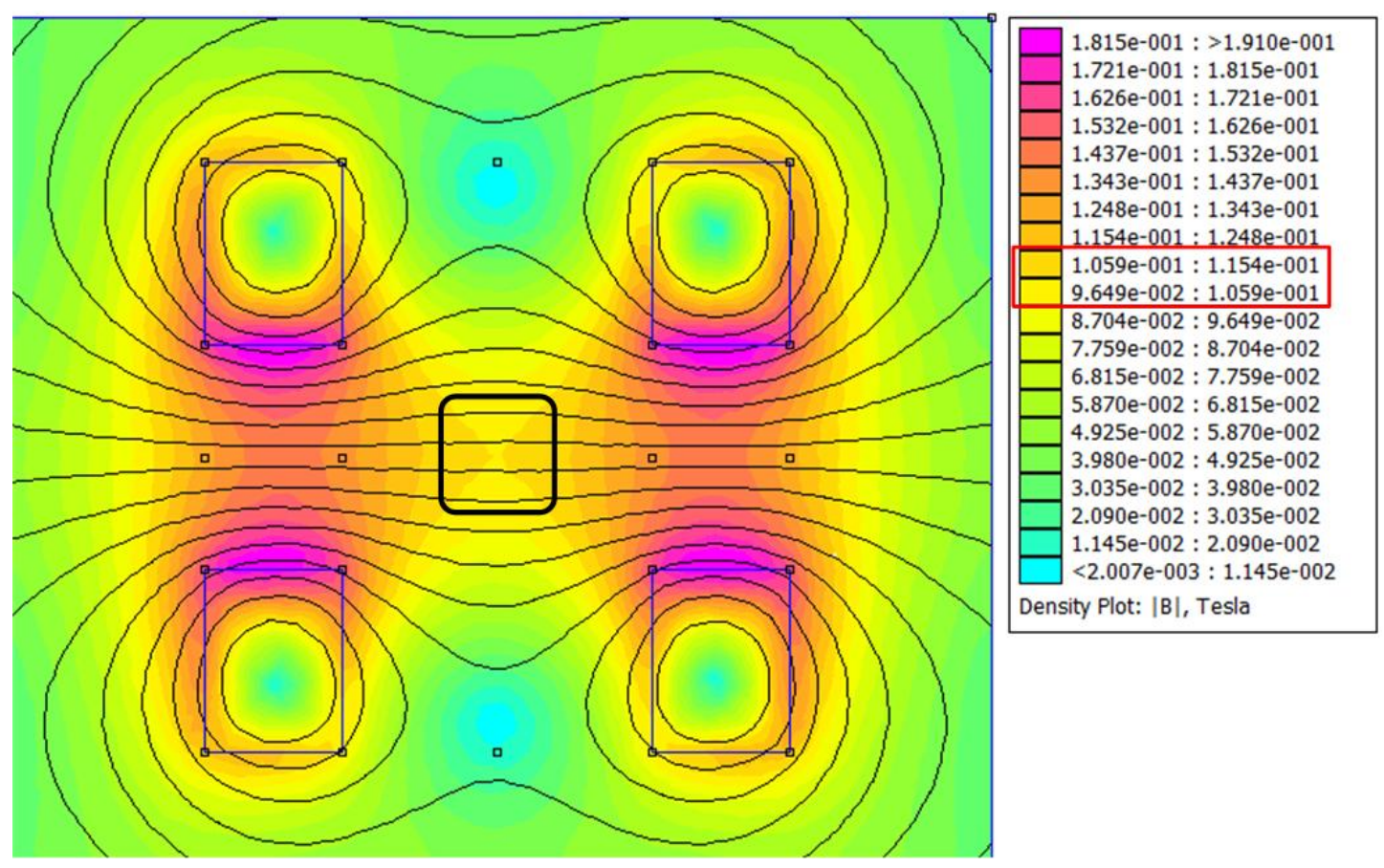

Abbildung 3.7: Simulation der Magnetspulen mit Feldlinien, Flussdichten (farbige Legende) und der Position des Probenhalters

Um für eine ausreichende elektrische Isolierung der einzelnen Windungen voneinander zu sorgen, wurde das Kupferrohr mit Schrumpfschlauch umhüllt. Die Wärme des stromdurchflossenen Leiters muss abgeführt werden, damit der Schrumpfschlauch nicht zerstört wird und es dadurch nicht zu einem Kurzschluss der Windungen kommen kann. Der Abtransport der anfallenden Abwärme wird durch eine Wasserkühlung der Kupferrohre realisiert. Dazu werden diese auf der gesamten Länge mit Wasser durchspühlt. Bei maximalem Spulenstrom erhitzt sich das Kühlwasser bereits nach einer halben Minute auf ca. $60{ }^{\circ} \mathrm{C}$. Diese Kühlleistung ist ausreichend, da das Magnetfeld immer nur für wenige Sekunden benötigt wird.

\subsubsection{Stromquelle}

Zur Zündung der Bögen werden der Probenhalter (Kathode), die Kreuzflanschkammer (Anode) und der Zünddraht (Zündanode) über Stromdurchführungen an eine Stromquelle von aixcon Powersystems angeschlossen. Diese speziell für Hochstrompulse entwickelte und auf die Anforderungen von Arc Precision abgestimmte Stromquelle wurde stromstabilisierend konstruiert. Die Stabilisierung wird durch eine Zweipunktregelung realisiert. Abbildung 3.8 zeigt die Schwingung der Stromstärke. Die Periodendauer zwischen zwei Stromspitzen beträgt ca. $50 \mu \mathrm{s}$, was einer Regelfrequenz von $200 \mathrm{kHz}$ entspricht. Während die Frequenz der Regelschwingung konstant bleibt, ist die Amplitude der Regelschwingung von der Höhe des zu regelnden Stromes abhängig, und liegt für 0,5kA bei 65 Ampere (12\%), für 3kA 
bei 250 Ampere $(8,3 \%)$. Die Entladungen sind jedoch um ein Vielfaches länger als die Periodendauer der Regelfrequenz.

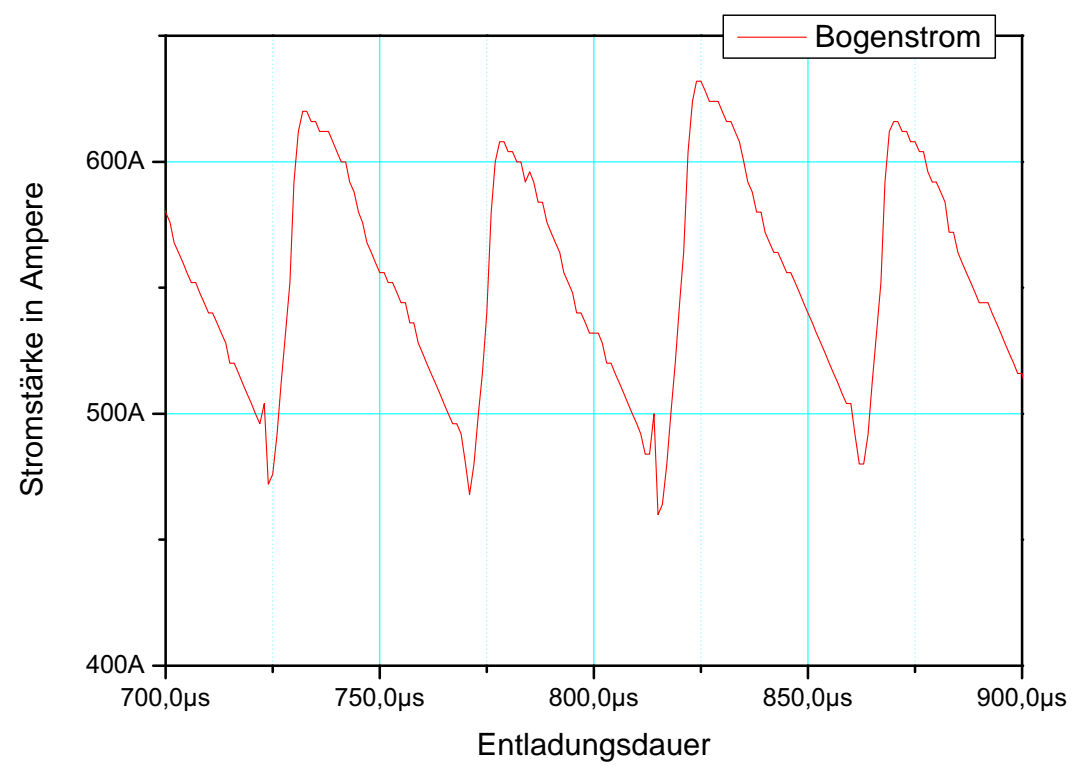

Abbildung 3.8: Regelschwingung der Stromquelle

Die Magnetspulen wurden an einer zweiten Stromquelle baugleicher Art angeschlossen.

\subsection{Optische Beobachtungstechnik}

\subsubsection{Hochgeschwindigkeitskamera - Phantom}

Zur detaillierten Beobachtung der Brennflecken bzw. der Brennfleckenfront wird vom IPP eine Hochgeschwindigkeitskamera bereit gestellt. (Phantom v7.3; Vison Research) Die Kamera verfügt über einen CMOS Sensor des Micro-Four-Third Formates (22 mm Sensordiagonale) mit einer Pixelbreite von $22 \mu \mathrm{m}$. Die Kamera ist mit dem Mikro Nikkor Objektiv von Nikkon ausgestattet, dessen Brennweite beträgt $60 \mathrm{~mm}$. Als allgemeiner Maßstab, für die im Verlauf der Versuche getätigten Aufnahmen, gilt die Größe von 80 bis $90 \mu m$ pro Bildpixel (Die optische Achse ist ca. $20 \mathrm{~cm}$ lang).

Bei höchster Aufösung (800 x 600 Pixel) kann die Kamera mit einer Bildwiederhohlungsrate (fps) von 10000 Bilder pro Sekunde Filme aufnehmen. Mit höherer fps bzw. geringerer Belichtungszeit (exp), muss die Aufösung der Kamera verringert werden. Bei der geringsten Auflösung von 8 x 32 Pixel können Aufnahmen von bis zu 250000 fps gemacht werden. Eine kleinere Auflösung, hat eine Einschränkung des Sichtfeldes der Kamera zur Folge. Es kann dann nur ein geringerer Teil der Entladung gefilmt werden.

Die Intensitätsauflösung der Kamera beträgt nominal 16 bit (ca. 65000 verschiedene Grauwerte) bzw. effektiv 14 bit (Grauwerte bis ca. 16000). 
Die Kamera wird zusammen mit der Phantom Camera Control Software v9.2 über einen PC betrieben. Zur Geschwindigkeitsmessung der Brennfleckenfront werden von der Software zwei Methoden bereitgestellt. Bei der Zweipunktmessung müssen zwei Punkte festgelegt werden. Die zweite Methode bezieht sich auf einen festen Punkt (origin) und einen jedes mal neu zu bestimmenden zweiten Punkt. Die vergangene Zeit ergibt sich bei beiden Methoden aus dem zeitlichen Abstand der jeweiligen Bilder, in denen die Punkte gewählt wurden.

\subsection{2 Übersichtskamera - Casio/EX-F1}

Eine zusätzliche Kamera bietet die Möglichkeit die Entladung, in voller räumlicher Ausdehnung, auch außerhalb des Bereiches den die Hochgeschwindigkeitskamera erfasst, zu betrachten und einzuschätzen. Um beide Kameras auf die Entladung zu richten, mussten sie mit einem geringen Winkel $(\alpha)$ zu Oberflächennormalen der Kathode positioniert werden. (siehe Versuchsaufbau Kapitel 4.2.1 auf Seite 26)

Bei der zweiten Kamera handelt es sich um eine Casio Exilim der Serie F1 mit 1/1.8 CMOS Sensor (Full HD, 2816 x 2112 Pixel bzw. 1920 x 1080 Pixel bei Videoaufnahmen). Das Objektiv hat eine Brennweite von 36 bis $432 \mathrm{~mm}$ und 12fach optischen Zoom. Sie kann 60 Halbbilder pro Sekunde aufnehmen (1200 fps) und liefert aus dem Videomaterial einzeln zu extrahierende Bilder, die eine Übersicht über die Entladung geben. Um eine Überbelichtung, durch die langen Belichtungszeiten, zu vermeiden wurden verschiedene Filter verwendet.

\subsection{Sonstige Messtechnik}

Zur Analyse der auf einer Festplatte abgeschiedenen Partikel im Durchmesserbereich 10 bis $500 \mathrm{~nm}$ wird die Candela 6100 (KLA-Tencor) verwendet. Sie erbringt den Nachweis und die Größenmessung durch Laserstreuung auf einem rotierendem Substrat.

Mit dem 4-Kanal Digitalspeicheroszilloskop (TDS2014B; Tektronix) können die elektrischen Parameter der Entladungen mit einer Auflösung von $100 \mathrm{MHz}$ beobachtet werden. Um den Zündimpuls aufzuzeichnen wurde eine Spannungsmessspitze an den Stromdurchführungen zum Probenhalter angebracht. Mittels Zangenstromwandlers (C160, Teilungsfaktor $1000 \mathrm{~A}$ x $1 \mathrm{mV} / \mathrm{A}$; Chauvin Arnoux) konnte auch der Bogenstrom von 500 Ampere bis 3000 Ampere aufgezeichnet werden.

Der magnetische Fluss wurde mit einem Digital-Tesla/Gausmeter (CYHT 201; Chen Yang Technologies) vermessen, bei einem statischen Magnetfeld liegt dessen Fehler bei $\pm 2,0 \%$. Um während des Ausheizens des Pumpstandes die Temperatur an den kritischen Stellen zu überwachen, werden Thermoelemte vom Typ K NiCr-Ni verwendet. (Toeranzklasse 1 $\pm 1,5{ }^{\circ} \mathrm{C}$ ) Die Thermoelemente sind über den Thermologger 309 von Voltcraft mit einem $\mathrm{PC}$ verbunden, und ermöglichen das Aufzeichnen der Temperaturverläufe. 


\subsection{Externe Möglichkeiten zur Probenuntersuchung}

Die Untersuchungen der Kathodenoberfläche erfolgte mit einem digitalen Lichtmikroskop (DLM) konfokaler Bauweise und einem EDX fähigen Raster Elektronen Mikroskop (REM). Die Nutzung beider Mikroskope wurde von der TH-Wildau gestattet. Das DLM ist wegen seiner hohen Tiefenschärfe in der Lage dreidimensionale Profile zu erstellen, aus denen die Tiefe der Kathodenerosion abgeschätzt werden kann. Das REM erlaubt aufgrund der energiedispersiven Röntgenspektralanalyse eine Aussage über die chemische Zusammensetzung der Proben.

Das IPP verfügt über ein automatisiertes Rasterelektronenmikroskop. Mit dessen Hilfen es möglich ist, Proben von großem Umfang und mit angemessener statistischer Genauigkeit zu untersuchen. 


\section{Kapitel 4}

\section{Durchführung der Experimente}

\subsection{Klassifizierung des Probenmaterials}

Als Kathodenmaterialien wurden vier verschiedene Proben genutzt, die bis auf Kohlenstoff vom IPP geliefert wurden.

Bezeichnung:

- C - Kohlenstoff

- W - Wolfram

- CW - Kohlenstoff mit einer $5 \mu m$ dicken Wolframschicht

- W $\mathrm{Al}_{2} \mathrm{O}_{3}$ - Wolfram mit einer $200 \mathrm{~nm}$ dicken Aluminiumoxidschicht

Die Kohlenstoffkathoden haben eine Dichte von $1.94 \mathrm{~g} / \mathrm{cm}^{3}$, die thermische Leitfähigkeit von $112 \frac{W}{(m \cdot K)}$ und eine durchschnittliche Reinheit von weniger als $2 \mathrm{ppm}$. Sie werden von Poco Specialty Materials geliefert.

Die verwendeten Festplatten, hatten einen Durchmesser von $65 \mathrm{~mm}$ und bestanden hauptsächlich aus Aluminium.

Des weiteren wurde ein Silizium-Wafer genutzt. Der Wafer hatte einen Durchmesser von $100 \mathrm{~mm}$, eine 111 Orientierung und ist Bor dotiert (P-leitend). Die Leitfähigkeit des Wafers beträgt $15 \Omega \mathrm{cm}$. Der Wafer wurde in unterschiedlich große Stücke geschnitten und zu den entsprechenden Experimenten in die Kreuzflanschkammer eingebaut.

Alle benutzen Proben sowie die Ordner im Archiv des Bildmaterials werden nach dem entsprechenden Versuch bezeichnet. Diese Versuchbezeichnung enthält Datum (nach ISO 8601, JJMMTT), Probenmaterial (Kurzbezeichnung) und ggf. eine Bemerkung zur Vorgehensweise. Zum Beispiel folgende:

- 101105 - C - Droplets

- 110107 - CW - Wafer N50

- 110127 - W 


\subsection{Aufbau und Durchführung der Versuche}

\subsubsection{Beobachtung der Brennfleckdynamik}
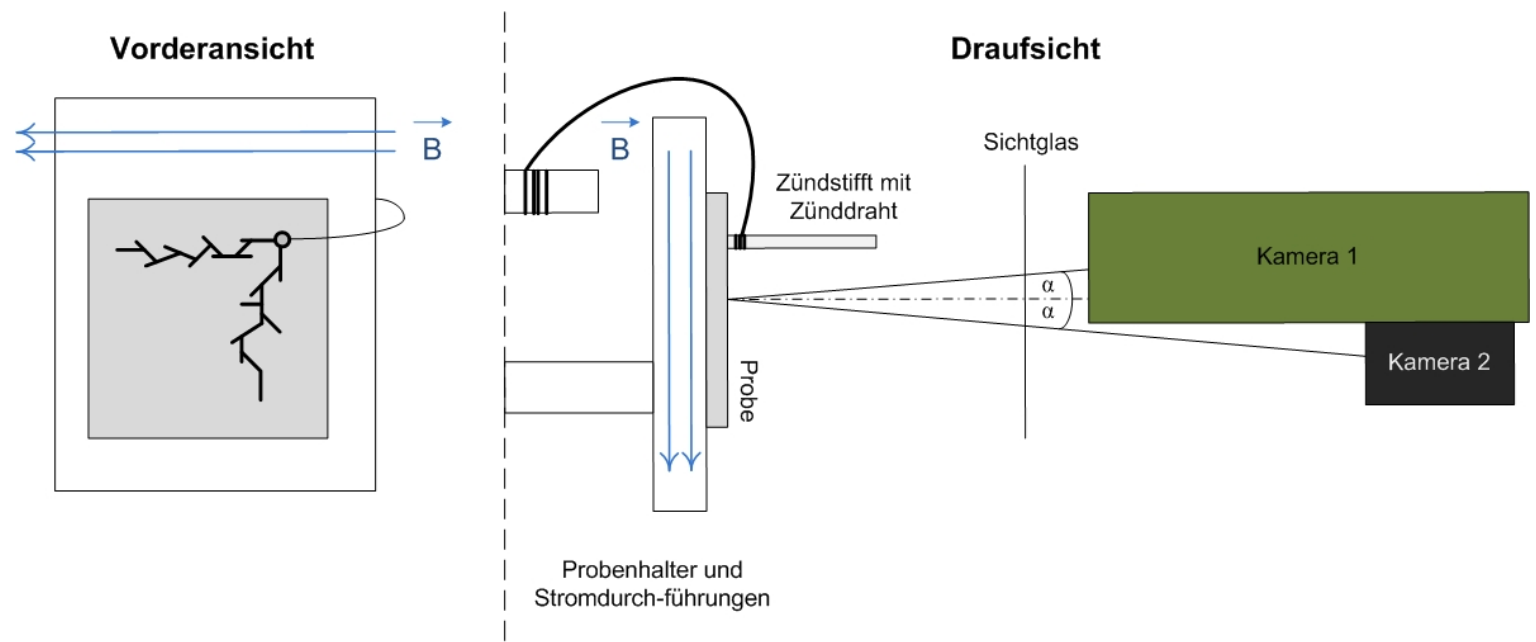

Abbildung 4.1: Versuchsanordnung zur Beobachtung der Brennfleckdynamik, Vorderansicht der Kathode links aus Sicht beider Kameras und Draufsicht rechts

Die Kathode, auf der sich der zu beobachtende Vakuumbogenbrennfleck bewegen soll, wurde wie in Abbildung 4.1 am Probenhalter befestigt. Die Bewegung des Brennfleckes auf der Kathode wurde durch das Sichtglas in der Kreuzflanschkammer mit Kamera 1, der Hochgeschwindigkeitskamera, beobachtet. Parallel zur Probenoberfläche kann ein möglichst homogenes magnetisches Feld erzeugt werden. Die Richtung des Feldes ist ebenfalls in Abbildung 4.1 eingetragen. Es wurden alle vier verschiedene Proben (C, W, CW, W $\mathrm{Al}_{2} \mathrm{O}_{3}$ ) untersucht.

Mit der Steuerungssoftware der Kamera lassen sich die Geschwindigkeiten der Brennflecken auf den verschiedenen Materialien, bei unterschiedlichen Magnetfeldern und Stromstärken messen bzw. auswerten.

Darüber hinaus kann mit Hilfe der Hochgeschwindigkeitskamera die Lebenszeit der Brennflecken abgeschätzt werden. Sie entspricht der Zeit, in denen sich der Brennfleck oder die Brennfleckfront nicht bewegt hat. Wobei die Genauigkeit der Abschätzung vom betrachteten Videomaterial abhängt.

Folgende Experimente wurden in Bezug auf Kohlenstoff, jeweils mit geringer, mittlerer und hoher Bildwiederholrate, durchgeführt und sind in Tabelle 4.1 aufgelistet.

Tabelle 4.1: Variation der Parameter magnetische Flussdichte und Bogenstrom bei den durchgeführten Experimenten zur Brennfleckdynamik auf Kohlenstoff

\begin{tabular}{cccc}
\hline Material & Datum & Bogenstrom in A & Flussdichte in $\mathrm{mT}$ \\
\hline Kohlenstoff & 21.10 .10 & 500 & 0 \\
& 18.10 .10 & 500 & $0 ; 12 ; 30$ \\
& $24 / 25.01 .11$ & 500 & 0 bis 150 \\
& $24 / 25.01 .11$ & 0,$5 ; 1 ; 3 ;$ & 0 \\
\hline
\end{tabular}


Tabelle 4.2: Durchgeführte Experimente zur Brennfleckdynamik bei unterschiedlichen magnetischen Flussdichten für $\mathrm{W} \mathrm{Al}_{2} \mathrm{O}_{3}$

\begin{tabular}{ccc}
\hline Material & Datum & Magnetfeld in $\mathrm{mT}$ \\
\hline $\mathrm{W} \mathrm{Al}_{2} \mathrm{O}_{3}$ & 28.12 .10 & 0 \\
& & 6 \\
& & 12 \\
& 23.12 .10 & 0 \\
\hline
\end{tabular}

Für alle anderen Materialien wurden die Versuche zur Brennfleckdynamik bei 500 Ampere Bogenstrom und geringeren Feldstärken durchgeführt. Tabelle 4.2 und 4.3 geben eine Auflistung. Des weiteren wurde jede Versuchsreihe nach Möglichkeit für beide Brennflecktypen durchgeführt.

Tabelle 4.3: Durchgeführte Experimente zur Brennfleckdynamik bei unterschiedlichen magnetischen Flussdichten für Wolfram, Wolfram beschichteten Kohlenstoff

\begin{tabular}{ccc}
\hline Material & Datum & Magnetfeld in $\mathrm{mT}$ \\
\hline Wolfram & 18.10 .10 & 0 \\
& & 12 \\
& 23.11 .10 & 0 \\
& & 6 \\
& $26 / 27.01 .11$ & 0 \\
& & 4 \\
& & 7 \\
& & 15 \\
Material & Datum & 20 \\
\hline Kohlenstoff & 25.11 .10 & 0 \\
Wolfram & & 4 \\
(CW) & & 7 \\
& 01.12 .10 & 0 \\
& & 4 \\
\hline
\end{tabular}




\subsubsection{Beobachtung der Partikelemission}

\section{Seitenansicht}

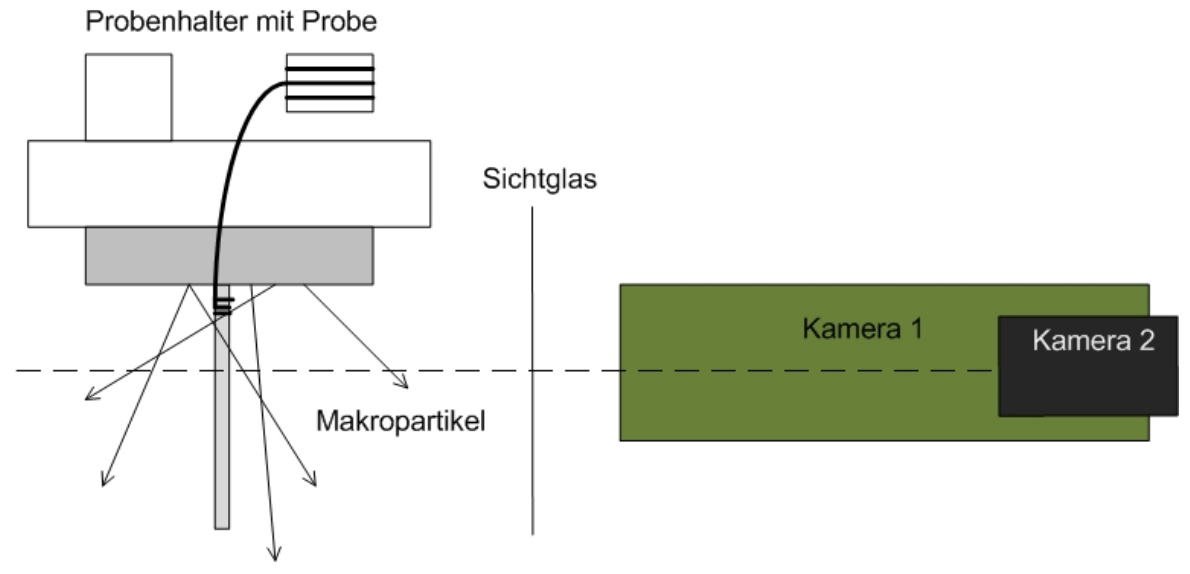

Abbildung 4.2: Versuchsanordnung zur Beobachtung der Makropartikel, Seitenansicht

Wie bereits in Kapitel 2.4 auf Seite 8 besprochen sind die Kathodenbrennflecken Quelle von Makropartikeln, die mit hoher Geschwindigkeit emittiert werden. Um diese näher charakterisieren zu können, werden sie mit zwei Methoden untersucht.

- Beobachtung der Flugbahn mit der Hochgeschwindigkeitskamera

- Auffangen der Makropartikel mit Silizium-Wafer und Festplatte

Mit der Hochgeschwindigkeitskamera bzw. der in Abbildung 4.2 dargestellten Versuchsanordnung kann die Flugbahn der Makropartikel beobachtet werden, nachdem sie vom Brennfleck emittiert wurden. Für diese Versuchsreihen wurden Entladungen von 0,5 bis 8 ms Brenndauer und Bogenströme von 500 Ampere sowie 1000 Ampere genutzt.

Kohlenstoff, beschichteter Kohlenstoff und Wolfram wurden als Proben gewählt. Alle Versuch mit massiven Wolframproben scheiterten am Versuchsaufbau (Fehlzündungen). Die Versuchsreihen sind in Tabelle 4.4 aufgelistet.

Für ein quantitatives Ergebnis der Versuche werden die sichtbaren Makropartikel gezählt bzw. deren Geschwindigkeit gemessen. Die Flugbahn der Makropartikel setzt sich aus drei Richtungskomponenten zusammen.

- Horizontal

- Vertikal

- Raumtiefe

Die Raumtiefenkomponente verfälscht das Ergebnis der Geschwindigkeitsmessung und soll daher möglichst klein sein. Deshalb werden die Versuche mit geöffneter Blende durchgeführt und nur die Makropartikel berücksichtigt, welche sich im selben Tiefenschärfebereich befinden und somit senkrecht zur optischen Achse des Kameraobjektives bewegen (kurzer Bereich der Schärfe $\Delta d$ zwischen Nah- und Fernpunkt, siehe Abbildung 5.47 in Kapitel 5.2 .4 auf Seite 64 Fehlerbetrachtung). 
Tabelle 4.4: Durchgeführte Experimente zum Auffangen der Makropartikel, Variation des Bogenstromes und der magnetischen Flussdichte

\begin{tabular}{cccc}
\hline Material & Datum & Bogenstrom & Magnetfeld \\
\hline Kohlenstoff & 04/05.11.10 & $500 \mathrm{~A}$ & $0 \mathrm{mT}$ \\
& $500 \mathrm{~A}$ & $12 \mathrm{mT}$ & \\
& $500 \mathrm{~A}$ & $30 \mathrm{mT}$ & \\
& $500 \mathrm{~A}$ & $50 \mathrm{mT}$ & \\
& $500 \mathrm{~A}$ & $70 \mathrm{mT}$ & \\
& $1 \mathrm{kA}$ & $12 \mathrm{mT}$ & \\
& $1 \mathrm{kA}$ & $50 \mathrm{mT}$ & \\
\hline Kohlenstoff & $1 \mathrm{kA}$ & $70 \mathrm{mT}$ & \\
-Wolfram & 29.11 .10 & $500 \mathrm{~A}$ & $0 \mathrm{mT}$ \\
& $500 \mathrm{~A}$ & $4 \mathrm{mT}$ & \\
\hline
\end{tabular}

Um die Makropartikel aufzufangen, wird ein Siliziumwafer bzw. eine Festplatte in ihre Flugbahn gebracht. Die Anordnung ist in Abbildung 4.3 skizziert. Die Wafer werden im Rasterelektronenmikroskop an der TH-Wildau und durch das IPP untersucht. Von Interesse ist hierbei die Größe, Struktur und Zusammensetzung der Makropartikel, sowie deren Verteilung auf dem Wafer. Die Festplatten werde mit der Candela 6100 vermessen. Die Oberflächendefekte werden gezählt, ihrer Größe nach in Klassen eingeteilt und deren Verteilung auf der Festplatte angegeben.


Abbildung 4.3: Experimentelle Anordnung zum Auffangen der Makropartikel, links Foto, rechts Skizze

Sieben Versuche wurden in dieser Anordnung durchgeführt. Tabelle 4.5 fasst sie zusammen. 


\begin{tabular}{ccccc}
\hline Datum & Detektor & Entladungen & Strom in A & Entladungsdauer in $\mu s$ \\
\hline 05.11 .10 & Si-Wafer & 1 & 500 & 500 \\
07.11 .10 & & 50 & - & - \\
10.11 .10 & & 500 & - & - \\
13.11 .10 & & - & - \\
19.11 .10 & & - & - \\
\hline 08.12 .10 & Festplatte & unbehandelt & & \\
08.12 .10 & & ein-/ausschleusen & & 300 \\
10.12 .10 & & 1 & 300 & \\
\hline
\end{tabular}

Tabelle 4.5: Durchgeführte Experimente zum Auffangen der Makropartikel; Variation der Anzahl der Entladungen und des Detektors

\subsubsection{Beobachtung der Kathodenerosion}

Der Versuchsaufbau entspricht hier dem in Kapitel 4.2.1 geschilderten. Nachdem die Proben durch die Entladungen erodiert wurden, folgte die Untersuchung mit einem digitalen Lichtmikroskop und einem Rasterelektronenmikroskop. Tabelle 4.6 listet die mit Hilfe der TH-Wildau untersuchten Proben auf. Darin werden die Proben (Datum, Material) den jeweiligen Parametern Bogenstrom, magnetische Flussdichte und Anzahl der Entladungen zugeordnet und das zur Untersuchung genutzte Verfahren angegeben. Weitere Proben, der gleichen Art, wurden dem IPP zur weiteren Untersuchung übergeben.

Tabelle 4.6: Durchgeführte Experimente zur Erosion der Kathodenoberfläche, Anzahl der Entladungen, Bogenstrom und magnetische Flussdichte als Parameter (zusammengefasst) und Untersuchungsmethode

\begin{tabular}{ccccc}
\hline Material & Datum & Entladungen & Parameter & Mikroskop \\
\hline Kohlenstoff & 05.11 .10 & $\begin{array}{c}\text { ca.100 } \\
12 \text { bis } 70 \mathrm{mT}\end{array}$ & 0,5 bis 1kA & DLM \\
\hline Material & Datum & Entladungen & Parameter & Mikroskop \\
\hline Wolfram & 23.11 .10 & 35 & 0 bis $11 \mathrm{mT}$ & DLM \\
\hline Material & Datum & Entladungen & Parameter & Mikroskop \\
\hline $\begin{array}{c}\text { Kohlenstoff- } \\
\text { Wolfram }\end{array}$ & $15 / 16.12 .10$ & $\begin{array}{c}1 ; 12 ; 51 \\
0 \mathrm{mT}\end{array}$ & $0,5 \mathrm{kA}$ & DLM \\
& 07.01 .11 & 50 & $0,5 \mathrm{kA}$ & REM \\
\hline Material & Datum & Entladungen & Parameter & Mikroskop \\
\hline $\mathrm{W} \mathrm{Al}_{2} \mathrm{O}_{3}$ & 28.12 .10 & $\begin{array}{c}19 \\
0\end{array}$ & \multicolumn{4}{c}{$0,5 \mathrm{kA}$} & DLM \\
\hline
\end{tabular}




\section{Kapitel 5}

\section{Ergebnisse}

Im ersten Teil des folgenden Kapitels 5.1 werden die Beobachtungen aller durchgeführten Versuche geschildert, diese sind nach dem Probenmaterial gegliedert.

- Kohlenstoff - C

- Wolfram - W

- Wolfram Kohlenstoff - CW

- Aluminiumoxid Wolfram - $\mathrm{WAl}_{2} \mathrm{O}_{3}$

Der zweite Teil dieses Kapitels 5.2 nimmt Bezug auf drei konkrete Punkte.

1. Strukturebenen der Bogenfußpunkte

2. Geschwindigkeit der Brennfleckfront und Einfluss eines externes Magnetfeldes

3. Zusammenhang zwischen Bogenstrom und Leuchtintensitäten der Brennflecken?

\subsection{Allgemeine Beobachtungen der Versuche}

\subsubsection{Kohlenstoff - C}

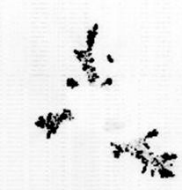

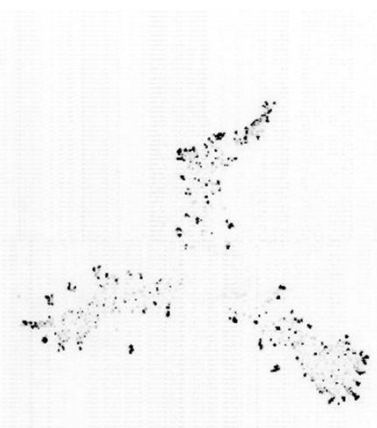



Abbildung 5.1: Kohlenstoffentladung (110124cine07 - C $I_{\text {Bogen }}=3 \mathrm{kA}, t_{\text {ent }}=500 \mu \mathrm{s}, B_{\text {ext }}=7 \mathrm{r}$ $m T)$, als Negativbild in der ersten Phase der Bewegung ( $t_{\text {interval }}=168 \mu \mathrm{s}$ exp $\left.=1 \mu \mathrm{s}\right)$

Vom Zündstifft beginnend breiten sich die hellen und diskret leuchtenden Brennflecken in radialer Richtung aus und überwinden dabei einige Millimeter bis Zentimeter Distanz auf 
der Probenoberfläche. (siehe Abbildung 5.1) Zu Beginn der Entladung ist die Ausbreitungsgeschwindigkeit der Brennfleckfront groß, sie nimmt jedoch rasch ab, bis die Brennflecken in der zweiten Phase der Entladung sich nicht mehr bewegen und stehen bleiben.
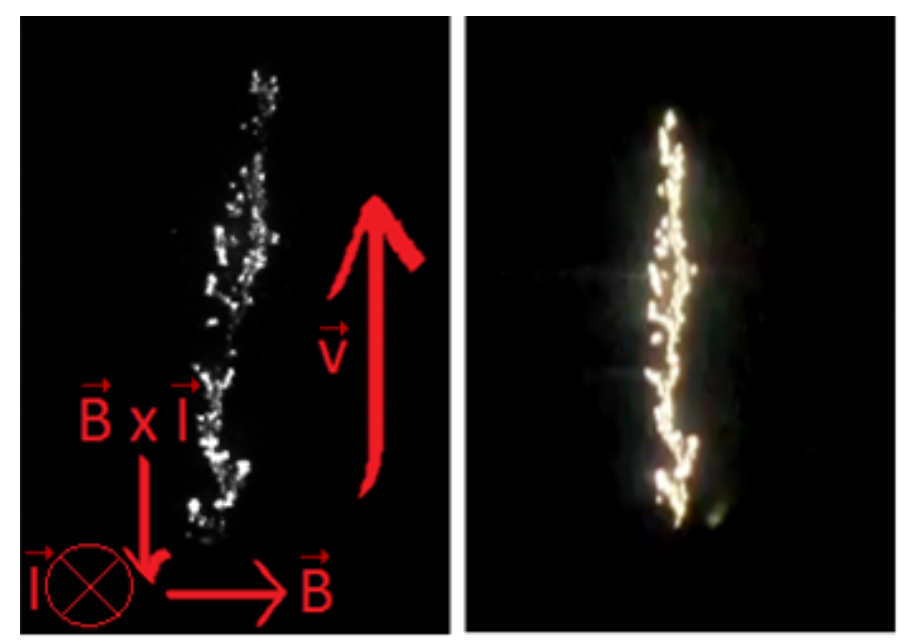

Abbildung 5.2: Kohlenstoffentladung (110124cine27 $-C I_{\text {Bogen }}=500 \mathrm{~A}, t_{\text {ent }}=500 \mu \mathrm{s}, B_{\text {ext }}=$ $77 \mathrm{mT}$ ), links kumuliert aus Kamera 1 (vier von acht Bildern $t_{\text {interval }}=44 \mu \mathrm{s} \exp =1 \mu \mathrm{s}$ ) und rechts als Übersicht aus Kamera 2

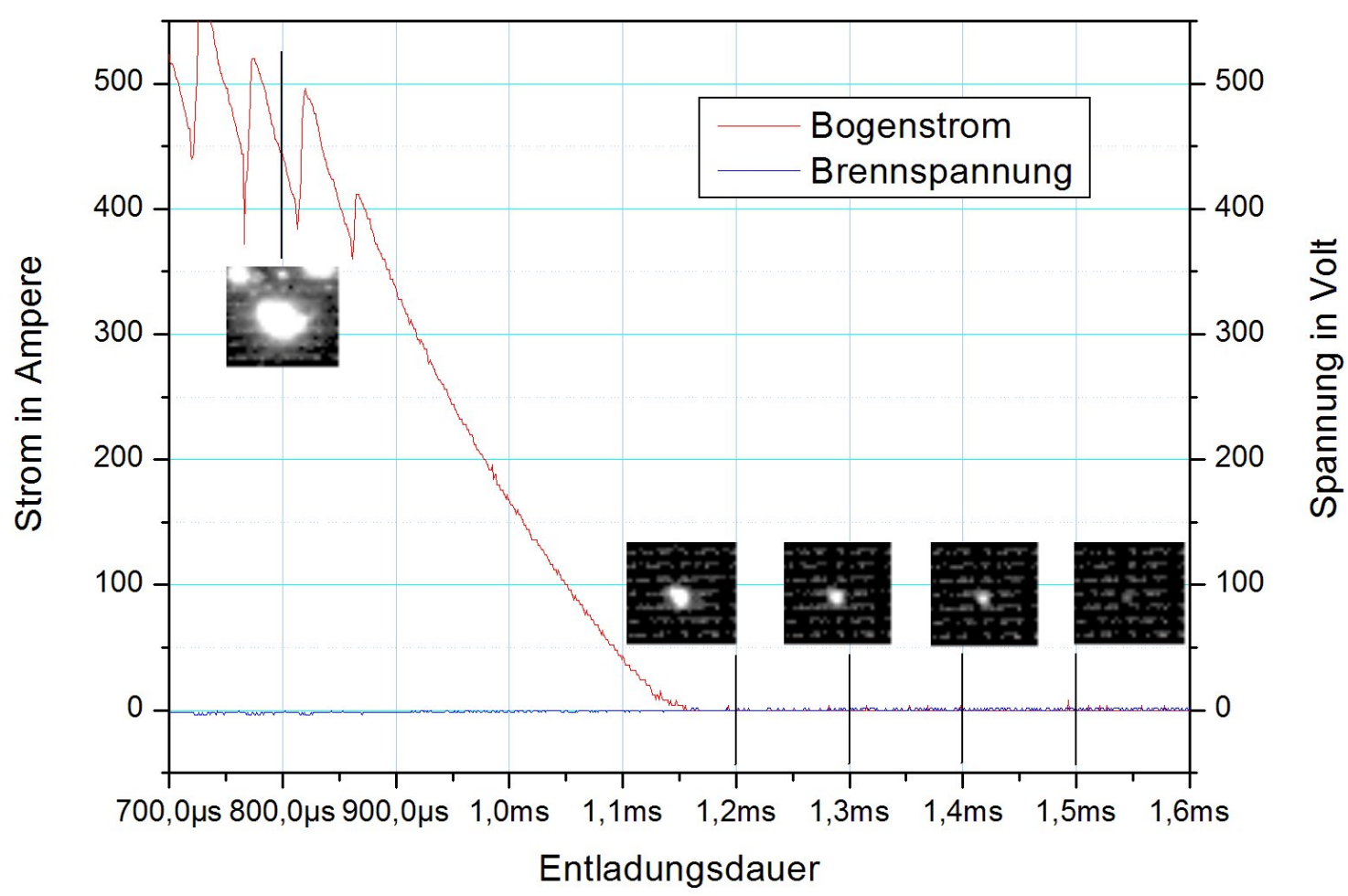

Abbildung 5.3: Bogenstromverlauf einer Kohlenstoffentladung (110124cine02-C $I_{\text {Bogen }}=500 \mathrm{~A}$, $t_{\text {ent }}=1 \mathrm{~ms}, B_{\text {ext }}=0 \mathrm{mT}$ ) mit fünf Aufnahmen der Entladung (verstärkt, $t_{\text {interval }}=20 \mu \mathrm{exp}=$ $1 \mu \mathrm{s})$, bei 0,8; 1,2; 1,3; 1,4; 1,5 ms nach dem Zündzeitpunkt

Ein externes Magnetfeld beeinflusst die Dynamik der Entladung erheblich. Die Brennflecken werden beschleunigt und bewegen sich Retrograd, der Lorentzkraft entgegen gerichtet, 
in diesem Fall (Abbildung 5.2 nach oben. In der Aufnahme sind die Ausbreitungsrichtung der Entladung (v), die konventionelle Stromrichtung (I), die Magnetfeldrichtung (B) und das Kreuzprodukt aus beiden letzteren eingezeichnet (I $x$ B).

Die Lebenszeit der Brennflecken auf Kohlenstoff beträgt mehrere hundert Mikrosekunden, was sie deutlich von den anderen Proben unterscheidet. Nach Ende der Entladung (Bogenstrom ist auf null Ampere gesunken) glühen die Stellen auf denen sich der Brennfleck als letztes aufhielt noch etwas nach. Abbildung 5.3 zeigt das Oszillogramm und fünf Aufnahmen einer Kohlenstoffentladung. Der Brennfleck erlischt erst nach ca. $1500 \mu s$, also $300 \mu s$ nach dem der Stromfluss beendet ist.

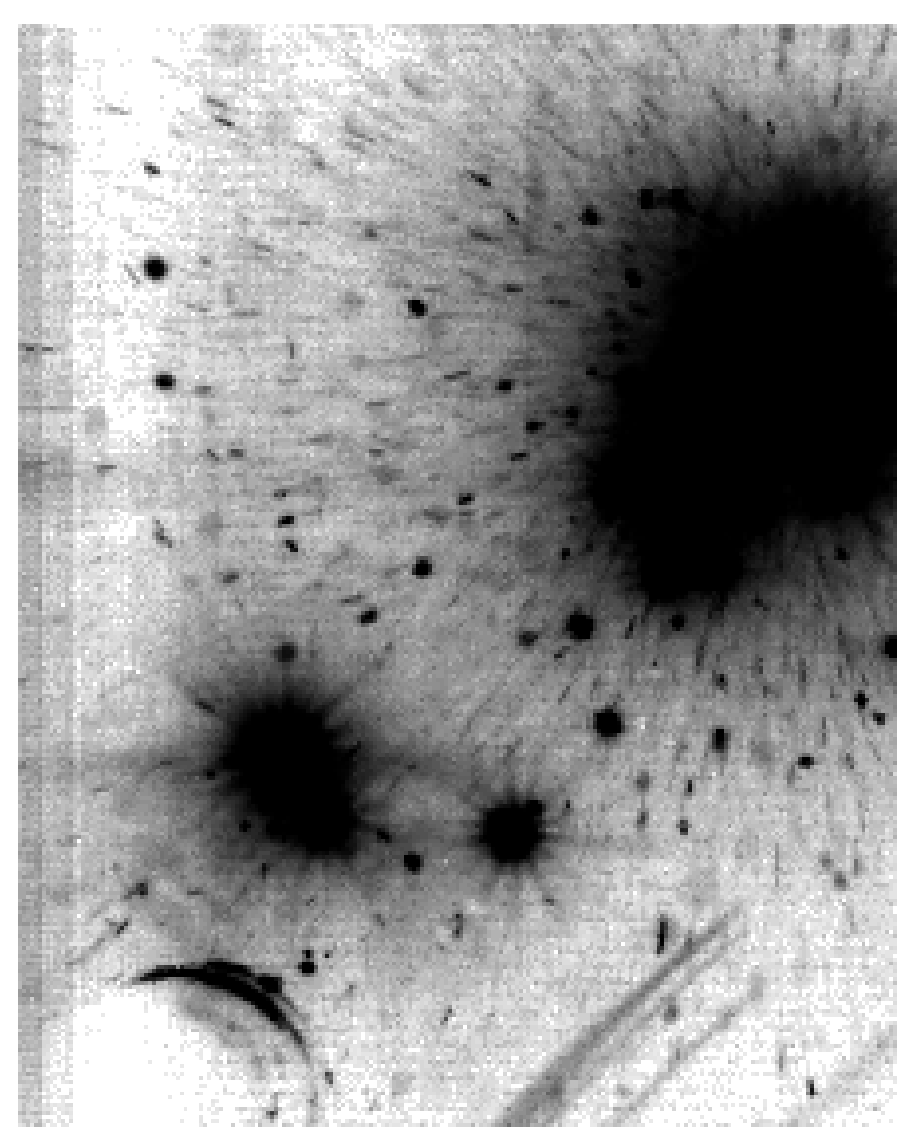

Abbildung 5.4: Negativbild einer Kohlenstoffentladung $\left(101118\right.$ cine06 $-C I_{\text {Bogen }}=500 \mathrm{~A}, t_{\text {ent }}=3 \mathrm{~ms}, B_{\text {ext }}=$ $11,1 \mathrm{mT})$ Vorderansicht, 64xFilter, Blende $16\left(t_{\text {interval }}=\right.$ $50 \mu$ exp $=9 \mu \mathrm{s})$; Makropartikel breiten sich in alle Richtungen aus
Über die Lebensdauer eines einzelnen Brennflecks hinweg kann beobachtet werden, dass dessen Helligkeit mit einer gewissen Frequenz schwankt. Es wird vermutet, dass diese Schwankung der Regelfrequenz der Stromquelle entspricht. Von dieser Fragestellung handelt Kapitel 5.2.3 auf Seite 58 .

Bei höherem Bogenstrom steigt die Zahl der Brennflecken bzw. Bogenspuren, nicht deren Intensität. Abbildung 5.1 zeigt eine Aufnahme 110124cine 07 bei $3 \mathrm{kA}$ Bogenstrom, es sind deutlich mehr Brennflecken zu erkennen als in Abbildung 5.2, bzw. drei Bogenspuren im Gegensatz zu einer Bogenspur.

Die Kohlenstoffprobe (101105 - C) wurde mit dem DLM untersucht, sie weist starke Erosion durch die Brennflecken auf. Eine typische und im Profil vermessene, Kraterspur hatte eine Tiefe von 40 $\mu \mathrm{m}$ und eine Breite von $99 \mu \mathrm{m}$. Die vermessene Probe wurde insgesamt von ca. 100 Entladungen mit Strömen bis $1 \mathrm{kA}$ und Magnetfeldern von bis $40 \mathrm{mT}$ belastet.

Die beobachteten und beschriebenen Brennflecken entsprechen eher dem in Kapitel 2.5 auf Seite 8 beschriebenem zweiten Typ, da ihre mittlere Geschwindigkeit relativ gering ist und die Oberflächenerosion groß ist. 



Abbildung 5.5: Emission von Makropartikeln aus einer Kohlenstoffkathode Seitenansicht, 6 fache Bildfolge (101105cine08 $-C I_{\text {Bogen }}=500 \mathrm{~A}, t_{\text {ent }}=1 \mathrm{~ms}, B_{\text {ext }}=3,4 \mathrm{mT}$, Bildabstand entspricht $t_{\text {interval }}=49 \mu \mathrm{s} \exp =19 \mu \mathrm{s}$

Abbildung 5.4 zeigt das Foto einer Entladung auf Kohlenstoff bei 500 Ampere Bogenstrom (101028cine06) in Vorderansicht und als Negativbild, das heißt die hell leuchtenden Brennflecken und Makropartikel sind in der Darstellung dunkel. Die Aufnahme entstand bei Blende 16 und einem 64-fachen Filter. Gut zu erkennen sind die von den Brennflecken ausgeworfenen Makropartikel, die während der gesamten Entladungsdauer emittiert werden.
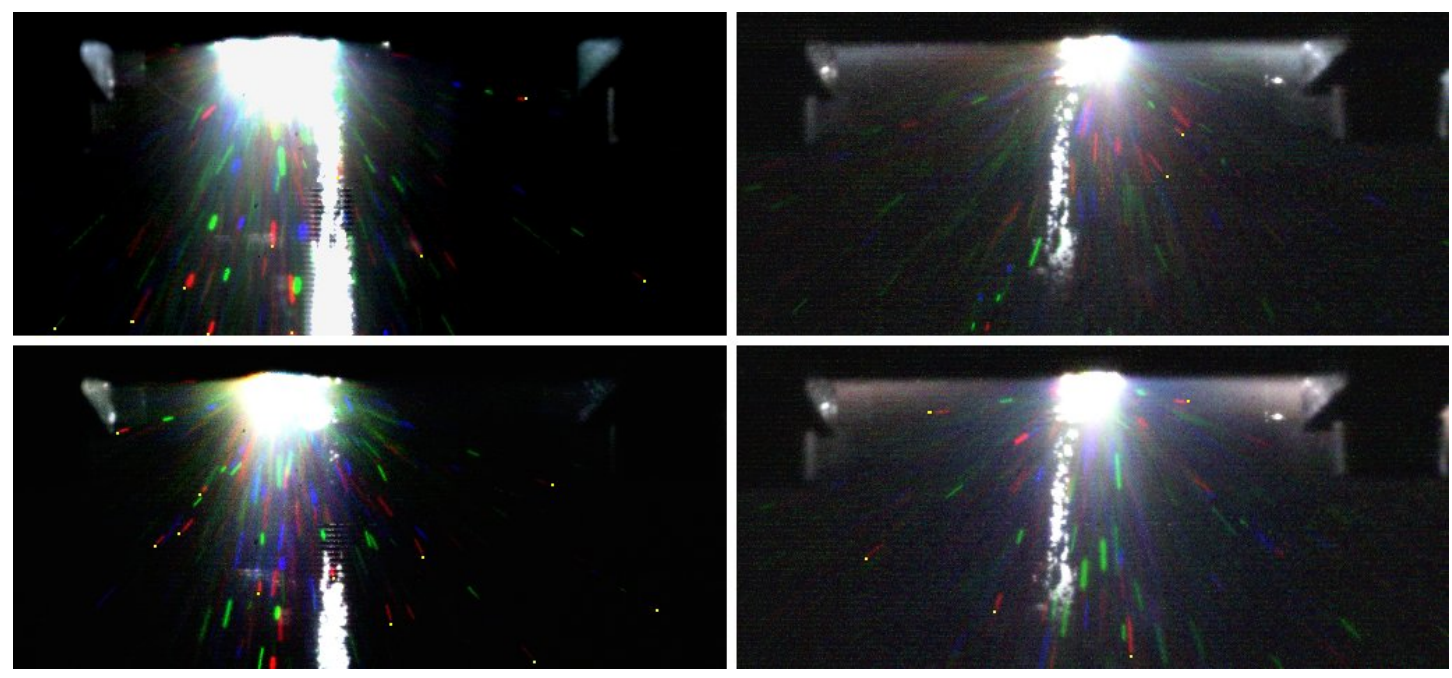

Abbildung 5.6: Emission von Makropartikel aus CW Kathoden RGB Kumuliert; zwei Entladungen unterschiedlich bearbeitetet (links und rechts) zu unterschiedlichen Zeitpunkten (oben und unten); gültige Makropartikel markiert (links: 101105 cine17a/b, rechts: 101105 cine27a/b alle $I_{\text {Bogen }}=$ $500 A$ )

In Relation zu den anderen Materialien bzw. Proben emittieren die Brennflecken auf Kohlenstoff die meisten sichtbaren Makropartikel (Durchschnittlich 7,3 bei Kohlenstoff gegen 4,2 auf beschichtetem Kohlenstoff).

Abbildung 5.5 zeigt vier Aufnahmen einer Entladung auf Kohlenstoff in der Seitenansicht. 
Die sichtbaren Makropartikel werden in alle Richtungen emittiert. Abbildung 5.6 zeigt vier Beispiele für die gesichteten Aufnahmen.

Es konnte ebenso keine Abhängigkeit der Auswurfrichtung der Makropartikel von einem externen Magnetfeld beobachtet werden.

Die Geschwindigkeit der sichtbaren Makropartikel liegt bei ca. 100 m/s (siehe Kapitel 5.2 .4 auf Seite 62 für genauere Daten).

\subsubsection{Wolfram - W}

Auf Wolfram konnten beide Brennflecktypen, sowohl Typ I als auch Typ II beobachtet werden. Sie sind in Abbildung 5.7 und 5.8 dargestellt. Die beiden Reihen setzen sich jeweils aus fünf hintereinander folgenden Bildern mit jeweils den selben Bildabständen zusammen. Die sechste Aufnahme der jeweiligen Reihe ist die Summe aller Einzelbilder.


Abbildung 5.7: Brennflecktyp I auf Wolfram (110127cine02 - W $I_{\text {Bogen }}=500 \mathrm{~A}, t_{\text {ent }}=500 \mu \mathrm{s}$, $\left.B_{\text {ext }}=6,2 m T, t_{\text {interval }}=50 \mu \mathrm{s} \exp =2 \mu \mathrm{s}\right)$

In Abbildung 5.7 ist der erste Brennflecktyp zu erkennen. Die durchschnittliche Schrittweite zwischen zwei Brennfleckfronten beträgt $3,3 \pm 0,3 \mathrm{~mm}$. Eine Schrittweite wird in $50 \mu \mathrm{s}$ zurückgelegt.
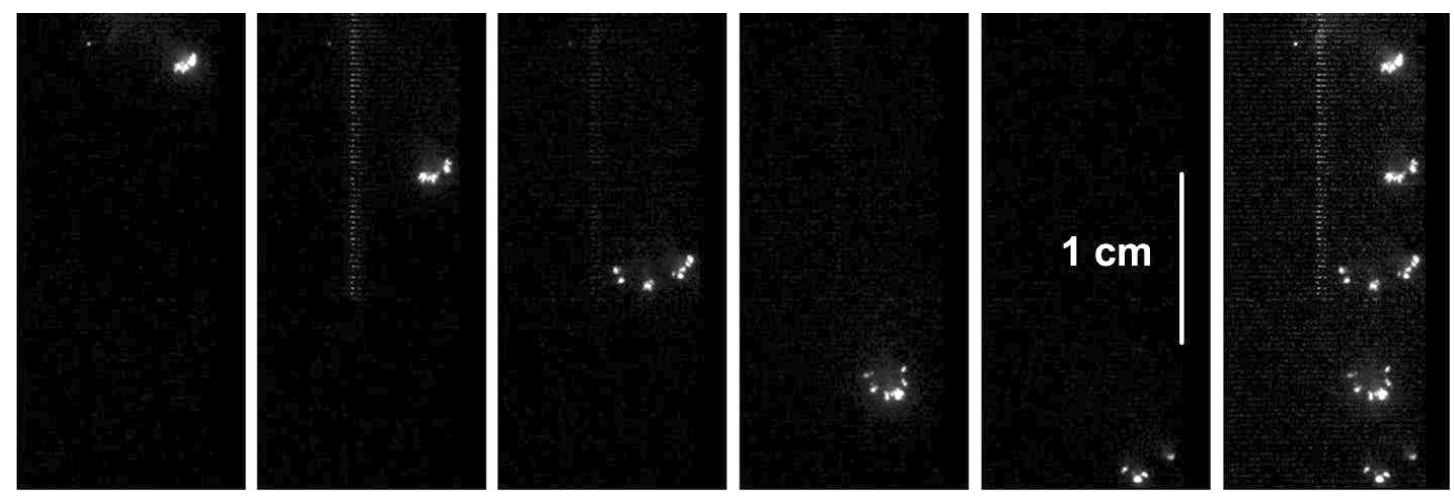

Abbildung 5.8: Brennflecktyp II auf Wolfram, stark verstärkt (110127cine11 - W $I_{\text {Bogen }}=500 \mathrm{~A}$, $\left.t_{\text {ent }}=500 \mu \mathrm{s}, B_{\text {ext }}=6,2 \mathrm{mT}, t_{\text {interval }}=50 \mu \mathrm{s} \exp =2 \mu \mathrm{s}\right)$

In Abbildung 5.8 ist der zweite Brennflecktyp zu erkennen, er bewegt sich schneller als der

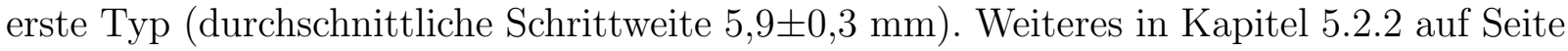


51 .
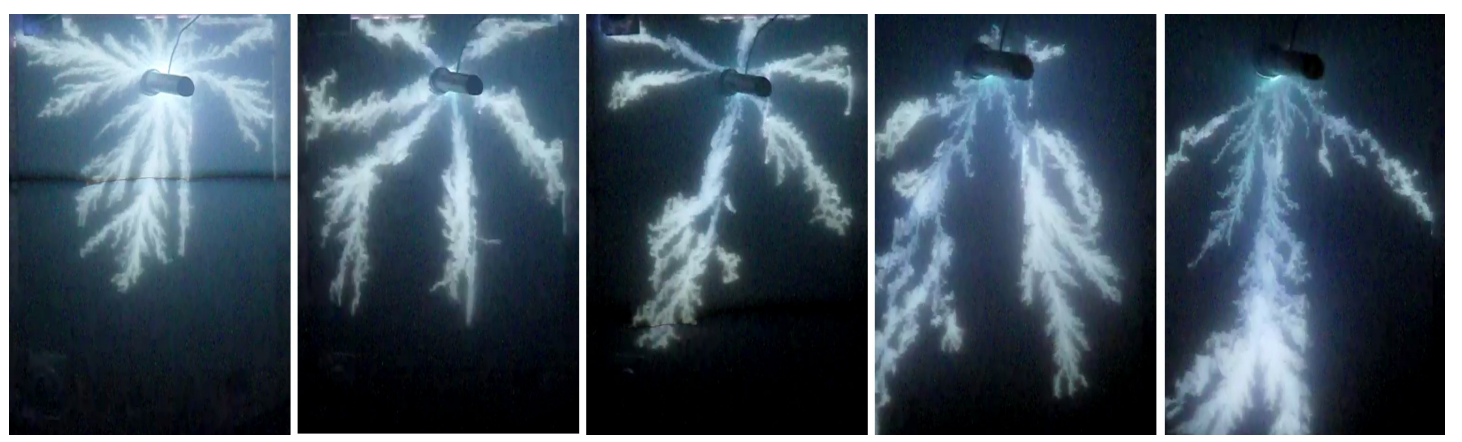

Abbildung 5.9: Fünf Entladungen hintereinander auf Wolfram (110126cine01/02/03 - W und 110127 cine01/02 $-W$ ) bei $I_{\text {Bogen }}=500 \mathrm{~A}, t_{\text {ent }}=500 \mu$ s v.l.n.r 0, 0, 0, 4, $7 \mathrm{mT}$;

Die Abbildung 5.9 zeigt fünf aufeinander folgende Entladungen. Alle Entladungen sind vom ersten Typ (N1 bis N5). Sie wurden mit Kamera 2 aufgenommen. Offenbar brennt die Entladung bevorzugt in kontaminierten Gebieten, dass heißt in bisher noch nicht von der Entladung erodierten Gebieten. Diese kontaminierten Gebiete oder Regionen sind bedeckt von Oberflächenverunreinigungen, wie $\mathrm{zu}$ Beispiel Gasen, Flüssigkeiten oder natürlichen Oxidschichten.

Um die Bevorzugung von kontaminierten Gebieten, und damit des ersten Typ's, nachzuweisen wurden die ersten drei Aufnahmen der Fotoreihe 5.9 addiert, unterschiedlich eingefärbt und in Abbildung 5.10 dargestellt.



Abbildung 5.10: RGB Kombination der ersten drei Aufnahmen aus Abbildung 5.9. (N1)Rot (N2)Blau (N3)Grün

In der Abbildung 5.10 ist $\mathrm{zu}$

erkennen, dass die ersten drei Entladungen sich kaum überlagern und dementsprechend, kaum Vermischungen der Farben auftreten.

Die Oberflächenuntersuchung mit dem DLM konnte keinen Schichtabtrag feststellen. Lediglich helle Spuren können nach 35 Entladungen auf dem Wolfram gesehen werden, sie 
sind in Abbildung 5.11 rechts abgebildet. Zusätzlich sind deutliche Verunreinigungen der Kathodenoberfläche als dunkle Spuren zu erkennen. Diese waren bereits vor den Entladungen auf der Oberfläche der Probe zu erkennen. Auf diesen Spuren brannte die Entladung immer wieder.
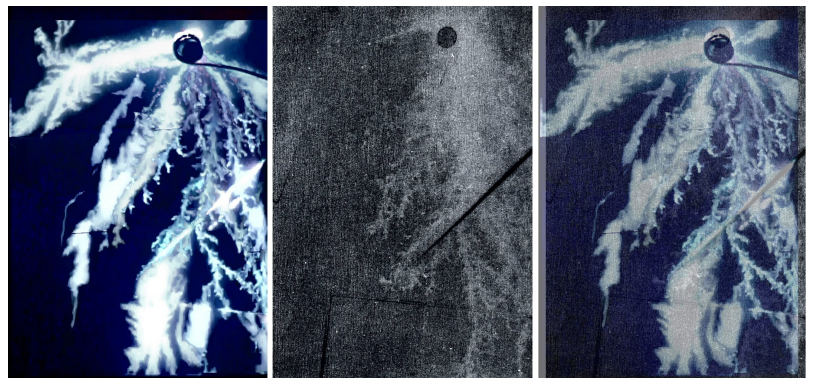

Abbildung 5.11: Links: Kumulierte Entladungsfotos von Kamera 2 nach 35 Entladungen (101123 - W: $I_{\text {Bogen }}=500 \mathrm{~A}, t_{\text {ent }}=500 \mu \mathrm{s}$ in Flussdichten von 0 bis $12 \mathrm{mT}$ ), Mitte: Entladungspuren auf Wolfram (helle Spuren: erosion durch Entladungen, dunkle Spuren: feste Verunreinigung bereits vor den Entladungen vorhanden) Rechts: beide Abbildungen übereinander

Aufgrund mangelnder Probenpräparierung (Fehlzündungen) konnten auf massiven Wolfram keine Makropartikel nach Versuchsaufbau ?? beobachtet werden. Die Geschwindigkeit der Brennfleckfronten auf Wolfram ist wesentlich höher als die der Brennflecken auf Kohlenstoff.

\subsubsection{Kohlenstoff - CW}

Auf den mit Wolfram beschichteten Kohlenstoffkathoden leuchten die Brennflecken des ersten Typs diffus und dunkel und die Brennflecken des zweiten Typs diskret und hell. Beide Typen sind in Abbildung 5.12 dargestellt, links Typ I und rechts Typ II.
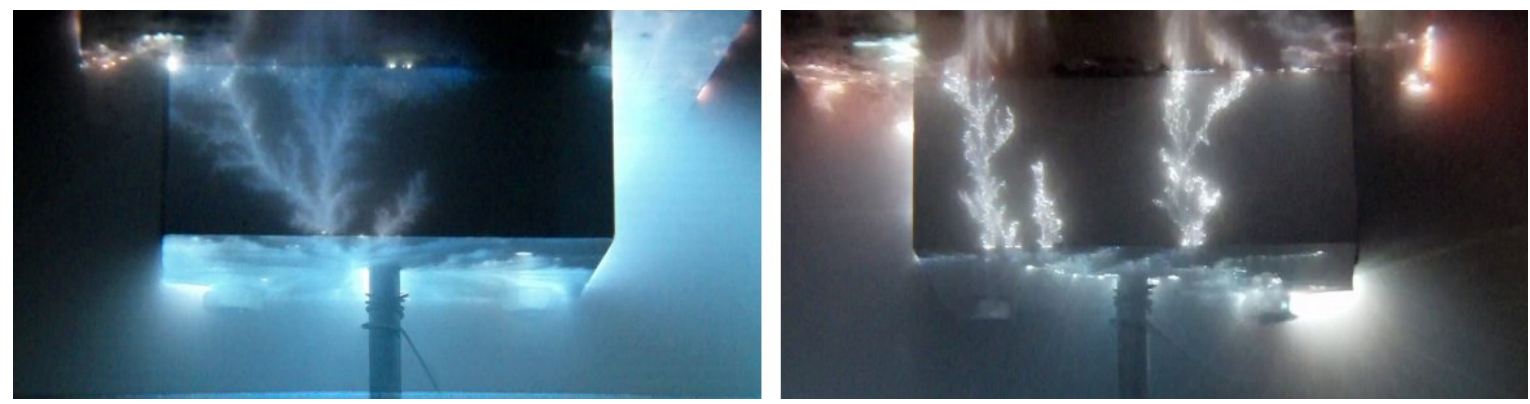

Abbildung 5.12: Links: Typ I erste Entladung; rechts: Typ II, 12. Entladungen, (101216 - CW Kathode $N 1 / N_{12} I_{\text {Bogen }}=500 \mathrm{~A}, t_{\text {ent }}=500 \mu \mathrm{s}, B_{\text {ext }}=0 \mathrm{mT}$ )

Die Aufnahmen der Hochgeschwindigkeitskamera sind für beide Typen in Abbildung 5.13 zu finden, wobei links Typ I und rechts Typ II zu erkennen ist. 

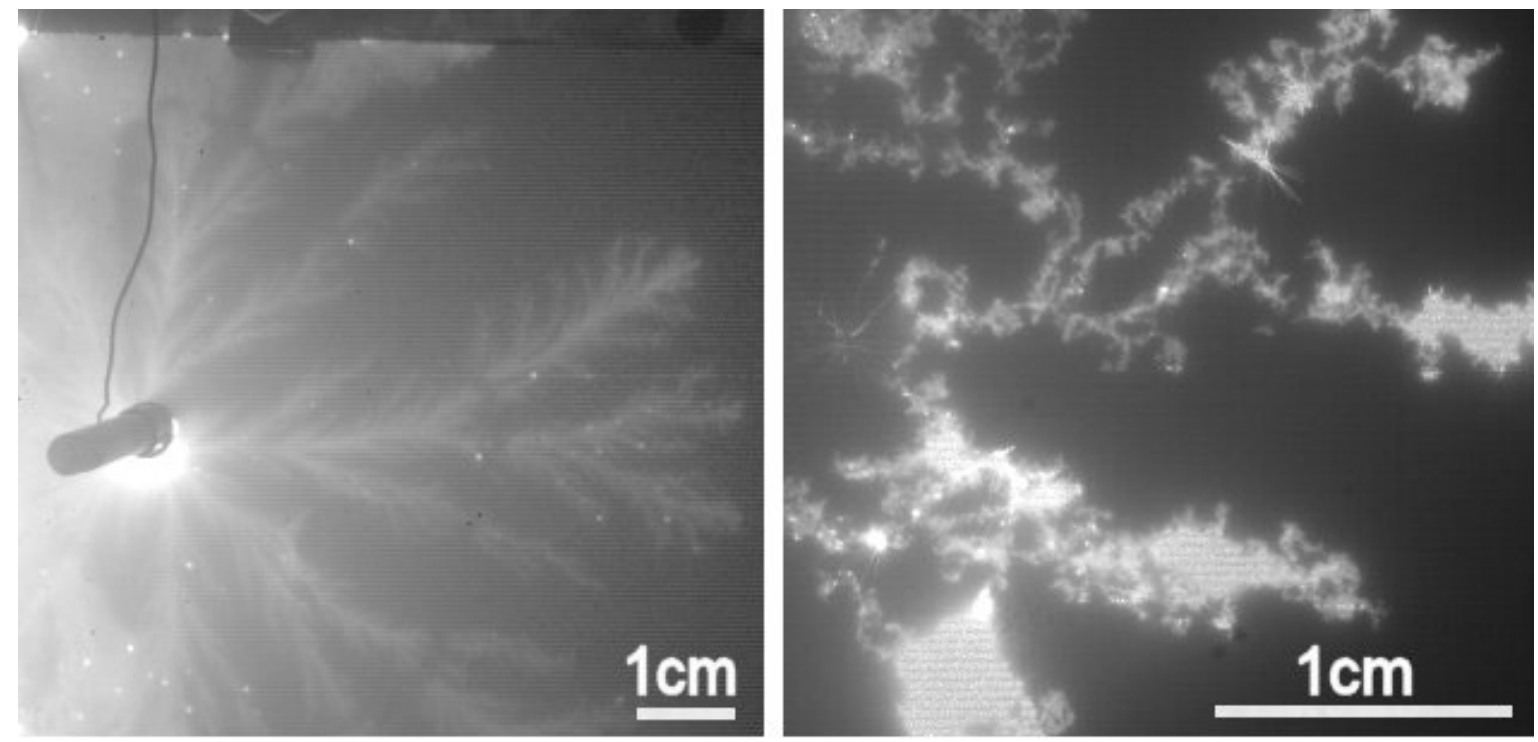

Abbildung 5.13: Links: erste Entladung - Typ I (11010\%cine01 - CW N1I Bogen $=500$ A, tent $=500 \mu \mathrm{s}, B_{\text {ext }}=0 \mathrm{mT}, t_{\text {interval }}=149 \mu \mathrm{s}$, exp $\left.=143 \mu \mathrm{s}\right)$ rechts: 7 . Entladungen - Typ II $\left(11010 \%\right.$ cine07- $C W N 7 I_{\text {Bogen }}=500 \mathrm{~A}, t_{\text {ent }}=300 \mu \mathrm{s}, B_{\text {ext }}=0 \mathrm{mT}, t_{\text {interval }}=45 \mu \mathrm{s}$, exp $=$ $42 \mu \mathrm{s})$; beide Aufnahmen aus zwei Bildern addiert
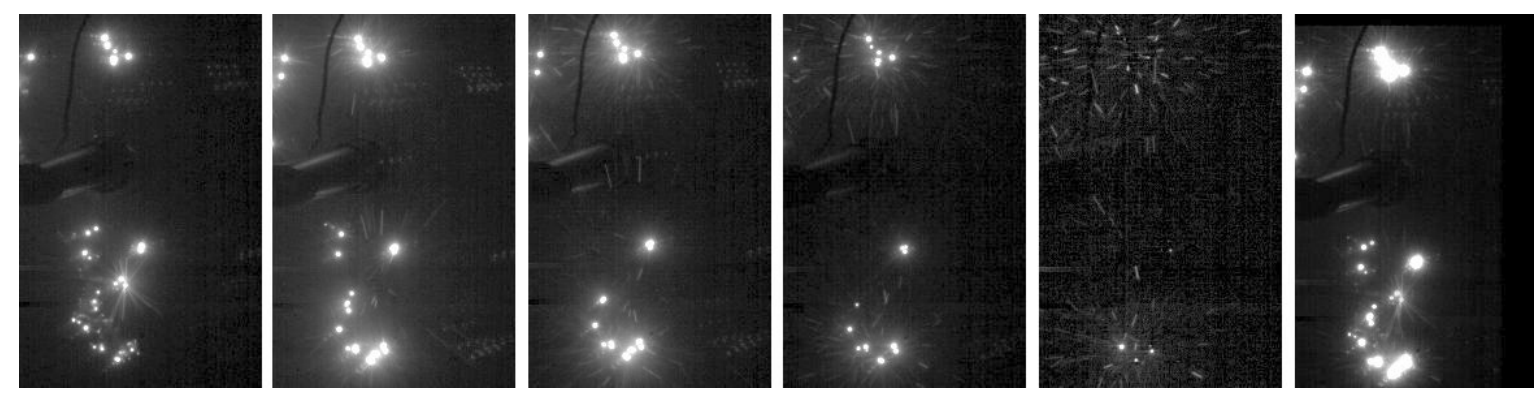

Abbildung 5.14: Kohlenstoff ähnliches Verhalten der Brennflecken (110107cine12- CW N41 I Bogen $=500 \mathrm{~A}, t_{\text {ent }}=300 \mu \mathrm{s}, B_{\text {ext }}=0 \mathrm{mT}, t_{\text {interval }}=98 \mu \mathrm{s}$, exp $\left.=50 \mu \mathrm{s}\right)$ Rechts: Summe aller Einzelbilder

Das Verhalten der Brennflecken entspricht zuerst dem von massiven Wolfram. Nach zehn bis zwanzig Entladungen auf der selben CW-Probenoberfläche schlägt das Verhalten der Entladung um, es entspricht nun eher dem Verhalten der Brennflecken auf Kohlenstoff. Wobei die Lebensdauer der Brennflecken sogar noch größer wird und teilweise der gesamten Entladungsdauer entspricht. Sie leuchten hell und befinden sich wiederholt an den selben Stellen, statt sich über die Oberfläche zu bewegen. Abbildung 5.14 stellt dieses Verhalten dar.

\section{Kathodenerosion}

Abbildung 5.15 links ist aus 300 Entladungen kumuliert, wobei jeweils hundert Entladungen addiert und dann einer der drei Farben rot, grün und blau zugewiesen wurden. An allen weißen Stellen brannte die Entladung demnach in jeder der drei Klassen (rot N200 N300; grün N300 - N400; blau N400 - N500) mindestens einmal. 

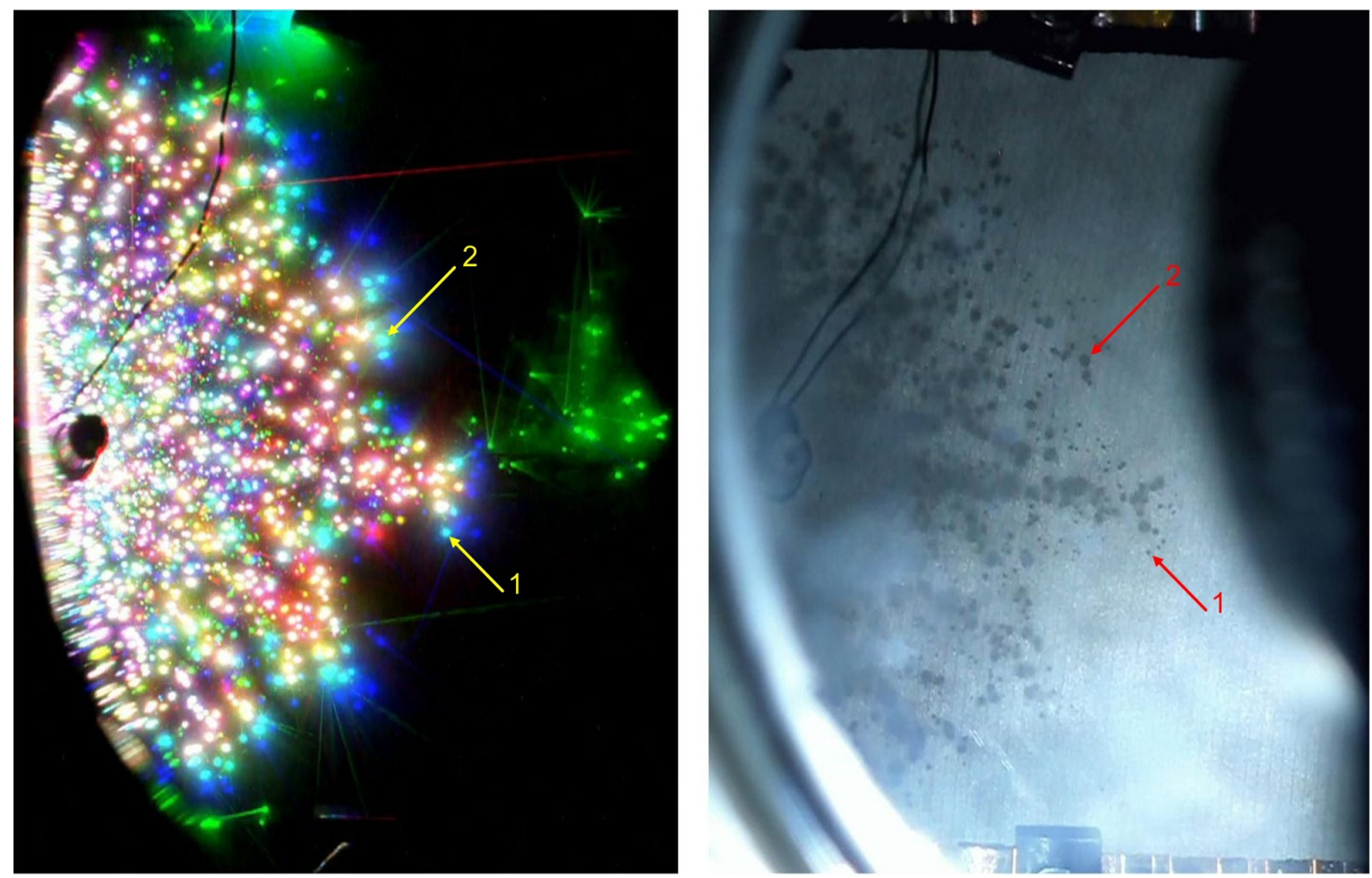

Abbildung 5.15: Zuordnung Brennflecken/Lochumgebung; Rechts RGB kumuliert aus Entladung 200 bis 500, auf beschichtetem Kohlenstoff (rot N200 - N300; grün N300 - N400; blau N400 N500); Links Probenoberfläche nach 500 Entladungen aus gleicher Perspektive, Brennfleckenmarkierung direkt übertragen (Versuchsreihe: 110113 - CW - Wafer N500)



Abbildung 5.16: Zuordnung Brennflecken/Löcher; Oben: RGB kumuliert aus Entladung 200 bis 500, auf beschichtetem Kohlenstoff; Unten: Aufnahmen mit Stereomikroskop (Versuchsreihe: 110113 - CW - Wafer N500); erstellt von Dr. rer. nat. P. Siemroth - Arc Precision 
Abbildung 5.15 rechts zeigt die selbe Probenoberfläche nach 500 Entladungen aus der selben Perspektive. Die Brennflecken können den dunklen Bereichen auf der Probenoberfläche (Lochumgebung) direkt zugeordnet werden. Abbildung 5.16 macht jedoch deutlich, dass die Brennflecken nicht den Löchern sondern nur deren Umgebung zugeordnet werden können.

Um den Einfluss der Brennflecken auf die Kathodenoberfläche genauer zu untersuchen, wurden Proben gleichen Materials mit unterschiedlich vielen Entladungen belastet und mikroskopisch untersucht. Beschichtete Kohlenstoffproben wurden gewählt (101216 - CW - N1/N12, bzw. 101215 - CW - N50) und mit einer, zwölf bzw. 50 Entladungen belastet. Übersichtsaufnahmen von allen drei Proben mit dem DLM sind in Abbildung 5.17 zu sehen. Der Unterschied in der Belastung der Proben ist deutlich zu erkennen. Die Probe N1 ist von Brennflecken des ersten Typs erodiert worden. Dieser Typ scheint großteils nur die Verunreinigungen der Oberfläche zu verbrennen, da er keine sichtbaren Spuren hinterlässt. Während die Probe N1 wenig erodiert ist, sind auf der Probe N50 mehrere Ansammlungen von Löchern (schwarze Punkte) zu sehen, so zum Beispiel auch in der roten Markierung in Abbildung 5.17. Diese ist mit zwei unterschiedlichen Vergrößerungen in Abbildung 5.18 links und rechts zu sehen.

N1



N12



N50

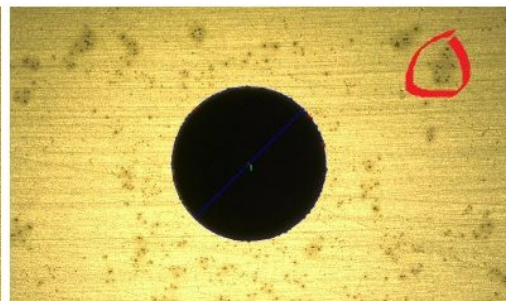

Abbildung 5.17: (v.l.n.r) CW nach einer, zwölf und 50 Entladungen unter dem DLM als Überblick; schwarzer Kreis: $3 \mathrm{~mm}$ Durchmesser (Zündstifftbohrung)
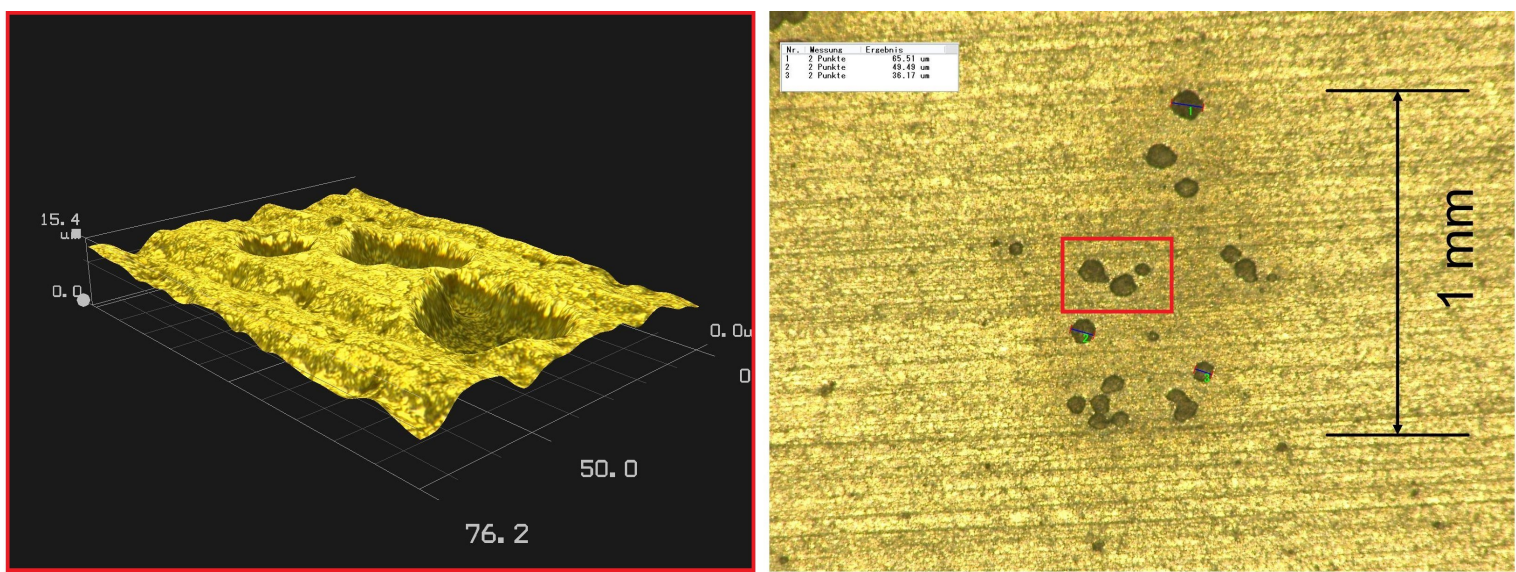

Abbildung 5.18: Links: Probe N50 bei starker Vergrößerung, entspricht der roten Markierung im rechten Teil des Bildes, die Abmessungen sind in $\mu$ m angegeben; rechts: Ansammlung der Löcher mit dunkler Umgebung, entspricht der roten Markierung in 5.17

Die Abbildung 5.18 rechts zeigt eine Ansammlung von Löchern und einen dunklen Bereich 
in deren Umgebung. Die Löcher haben einen Durchmesser von ca. 15 bis $65 \mu m$. Die Tiefe eines der Löcher konnte am DLM bestimmt werden und beträgt ca. $15 \mu \mathrm{m}$. Die $5 \mu \mathrm{m}$ dicke Wolframschicht wird also an dieser Stelle durchbrochen. Einige Löcher greifen ineinander über, was den Eindruck entstehen lässt, dass sie der bevorzugte Ausgangspunkt für neue Löcher sind. Das aber konnte bisher nicht nachgewiesen werden.

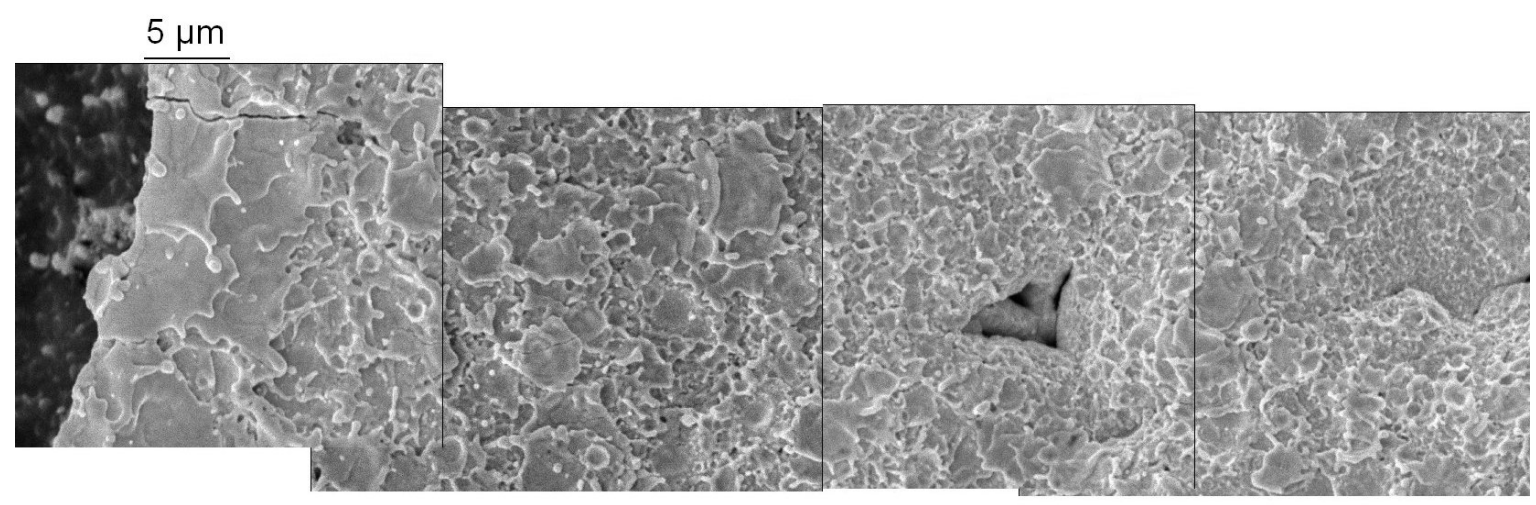

Abbildung 5.19: Lochrand mit belasteter Umgebung nach 50 Entladungen auf CW, Kohlenstoff schwarz (Loch), Wolframoberfläsche grau (Krater)

Die Löcher und ihre Umgebung wurden des weiteren im REM untersucht. Abbildung 5.19 (10107 - CW - N50) zeigt den Rand eines mit $100 \mu m$ Lochdurchmesser relativ großen Loches und seiner Umgebung. Die beeinflusste Zone hat einen ca. sechs mal größeren Durchmesser als das Loch und ist durch viele kleine, sich überlappende Krater, ähnlich denen in Abbildung 2.4, gekennzeichnet. Am Kraterrand sind Risse zu erkennen, die auf thermische Spannungen schließen lassen. Unbelastetes Gebiet ist dagegen in Abbildung 5.20 zu sehen.



Abbildung 5.20: Links: erodiertes Gebiet auf CW nach 50 Entladungen; Rechts: unbelastetes Gebiet (Typ I) auf der selben Probe 


\section{Partikelemission}

Die Beobachtung der Partikelemission wurden, wie in Kapitel 4.2 .2 auf Seite 28 beschrieben durchgeführt. Abbildung 5.21 zeigt, die Emission eines einzelnen Brennfleckenensembles (roter Pfeil).



Abbildung 5.21: Emission von Makropartikel aus CW Kathoden in fünffacher Bildfolge; oben links: Kennzeichnung von Kathode (grauer Kasten), Zündstifft (weißer Kasten) und Brennfleck (roter Pfeil); unten rechts: zweifach RGB kumuliert aus sechs Fotos, gültige Makropartikel gelb markiert $\left(101129\right.$ cine13arc04 $-C W I_{\text {Bogen }}=500 \mathrm{~A}, t_{\text {ent }}=500 \mu \mathrm{s}, B_{\text {ext }}=3,4 \mathrm{mT}, t_{\text {interval }}=100 \mu \mathrm{s}$ exp $=40 \mu \mathrm{s})$

Den gesichteten Aufnahmen ist, wie schon bei Kohlenstoffkathoden, keine bevorzugte Auswurfrichtung der sichtbaren Makropartikel zu entnehmen. Auch die in der Literatur 9 beschrieben Bevorzugung von kleinen Auswurfwinkeln kann nicht bestätigt werden. Für den Fall, dass die Löcher in der Kathodenoberfläche die hauptsächliche Quelle der Makropartikel sind, ist eine mögliche Erklärung Auswurfwinkel die Abschattung kleiner Winkel durch die Tiefe der Löcher und deren Rand.

Die Geschwindigkeit der sichtbaren Makropartikel liegt bei ca. 50 m/s (siehe Kapitel 5.2.4 auf Seite 62 für genauere Daten).

Die Auszählung von Makropartikel bei verändertem Bogenstrom lässt keine Aussagen zu.

Es wurde kein Einfluss des externem Magnetfeld auf die Emission von Makropartikeln festgestellt. 
Abbildung 5.22 zeigt vier Beispiele für die gesichteten Aufnahmen.


Abbildung 5.22: Emission von Makropartikel aus CW Kathoden (Beispiele), vier einzelne Entladungen unterschiedlich bearbeitete, jeweils aus drei Einzelbildern errechnet, gültige Makropartikel gelb markiert (von links nach recht und oben nach unten: 101129cine05/06/07/13 $I_{\text {Bogen }}=500 \mathrm{~A}$ $t_{\text {ent }}=500 \mu \mathrm{s}, B_{\text {ext }}=3,4 \mathrm{mT}$ für cine07/13)

Auf dem Silizium-Wafer (110113 - CW - Wafer N500) wurden viele Fremdpartikel und wenige kleine kugelförmige Partikel gefunden. (Abbildung 5.23 rechts). Eines dieser kugelförmigen Partikel wurde mit dem energiedispersiven Röntgenspektrometer analysiert und als Wolfram identifiziert.
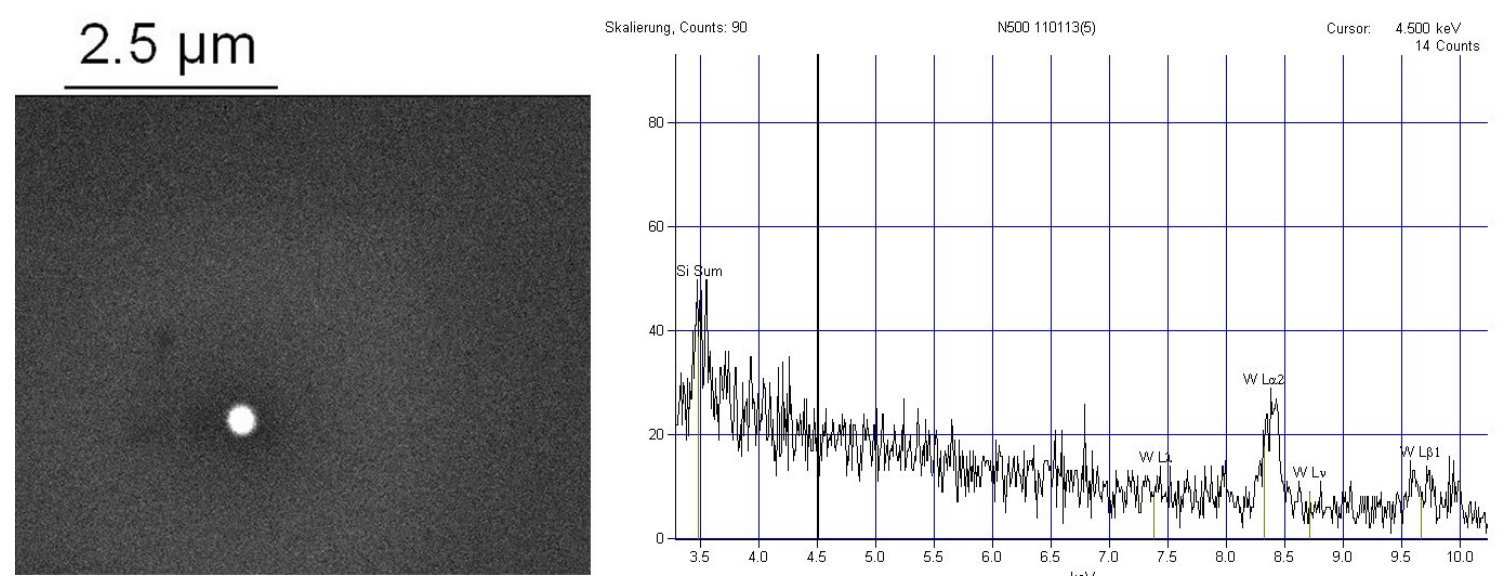

Abbildung 5.23: Links: Wolfram Droplet auf Silizium-Wafer aufgenommen im Rem(110113 - CW - Wafer N500) ; Rechts EDX-Spektrum des Wolfram Droplets

Die Größe des Partikels liegt bei ca. 350 nm. Wolfram konnte nur in dieser Form auf dem Wafer nachgewiesen werden, alle anderen der untersuchten Partikel enthielten kein Wolfram. Kohlenstoff konnte auf dem Wafer nicht gefunden werden. 
Aufgrund der Vielzahl von Partikeln konnten jedoch nur verhältnismäßig wenige davon untersucht werden. Dem entsprechend kann auch keine Verteilung bestimmter Makropartikel auf dem Wafer angegeben werden. Die Wechselwirkungsbirne des Elektronenstrahls hat ungefähr einen Mikrometer Durchmesser, sie erfasst also außer den zu untersuchenden Partikeln auch deren Umgebung. Die Siliziumlinien im EDX Spektrum sind darauf zurück zuführen. Das Rasterelektronenmikroskop an der TH-Wildau bot die Möglichkeit Proben mit einem Durchmesser von $25 \mathrm{~mm}$ zu untersuchen. Aus diesem Grund wurden die langen Wafer (Probe 110119 - CW - Wafer N500) an das Max-Planck-Institut für Plasmaphysik übergeben.

Die Partikelanalyse auf den Festplatten ergaben folgende Größenverteilung der emittierten Makropartikel.

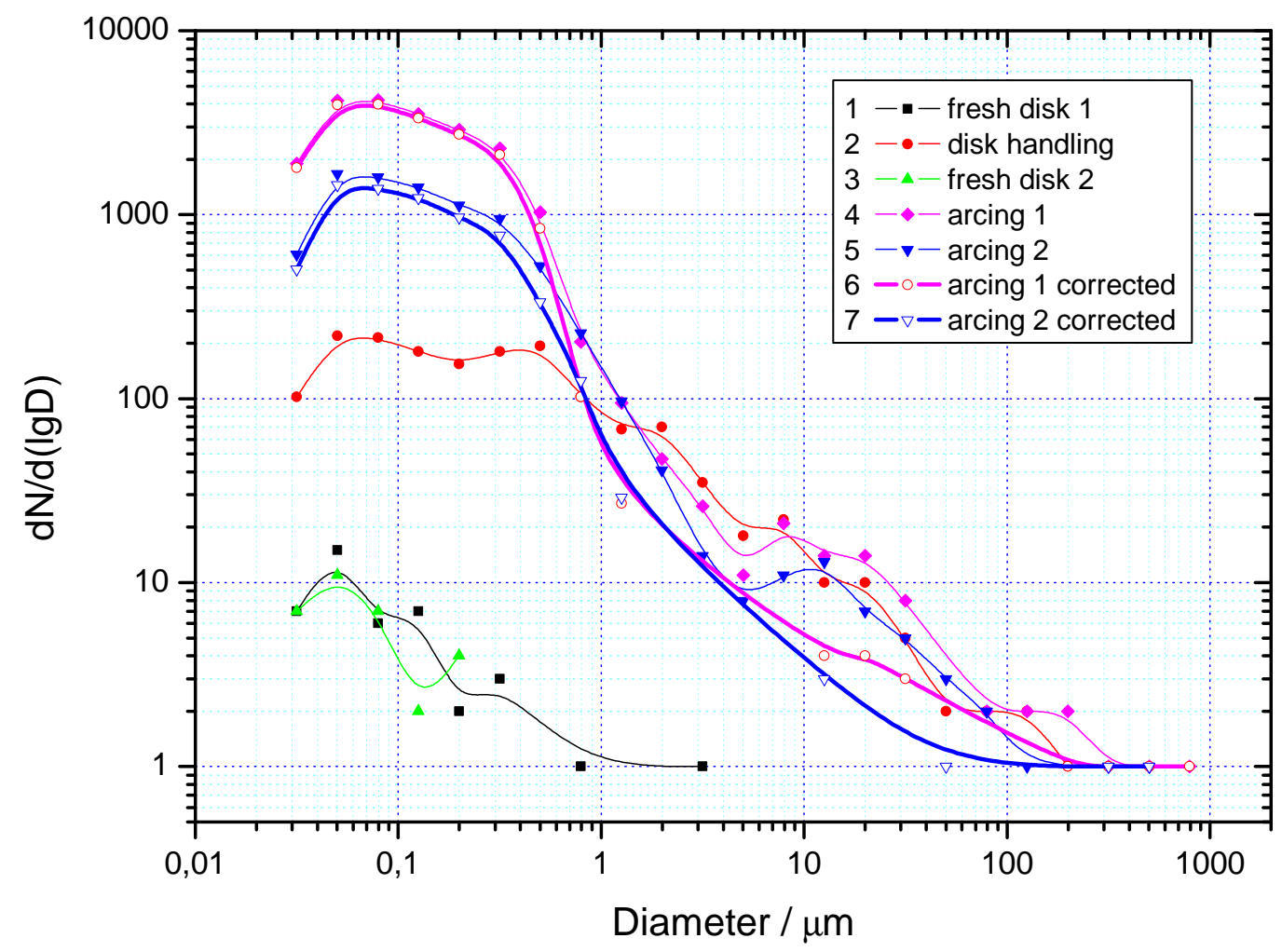

Abbildung 5.24: Ergebnisse der Partikelanalyse mit Candela 6100

Kurven 1 und 3 zeigen die Partikelzahlen und deren Größenverteilung bei Festplatten, direkt nach Entnahme aus dem Transportbehälter. Kurve 2 zeigt die Größenverteilung der Partikel auf einer Festplatte, die in die Versuchsanlage eingeschleust und nach kurzem Vakuumpumpen wieder ausgeschleust wurde. Kurven 4 und 5 zeigen die Größenverteilungen der Partikel auf zwei Platten, die in den Versuchen 10121001 und 02 mit einer Bogenentladung exponiert wurden. Kurven 6, 7 stellt den reinen Bogeneffekt durch Abzug der beim Handling applizierten Partikel (Kurve 3) dar (erstellt von Dr. rer. nat. P. Siemroth - Arc Precision)

„Die gefundene Verteilung oberhalb von $0,5 \mu \mathrm{m}$ fällt sehr steil ab, was im Gegensatz zu 
Messungen anderer Autoren (Anders, Daalder; ... ) steht. Eine mögliche Erklärung ist, dass jeweils nur eine einzige Entladung gemacht wurde und diese mit ziemlicher Sicherheit vom Typ 1 war, während frühere Untersuchungen zu Partikelgrößen immer mit gut formierten Kathoden ausgeführt wurden. Problematisch war, dass die extrem empfindliche CandelaAnlage schon bei den Partikeln einer einzigen Entladung hinsichtlich der Zählung an ihre Grenzen kam. Deshalb konnten die Untersuchungen nicht vertieft werden." Beschrieben durch Dr. rer. nat. P. Siemroth - Arc Precision.

Die lokale Verteilung auf der Festplatte war homogen.

\subsubsection{Wolfram - $\mathrm{WAl}_{2} \mathrm{O}_{3}$}

Als viertes Proben- bzw. Kathodenmaterial wurde beschichtetes Wolfram untersucht. Das Wolfram ist mit einer $200 \mathrm{~nm}$ dicken Aluminiumoxidschicht $\left(\mathrm{Al}_{2} \mathrm{O}_{3}\right)$ bedeckt. Aluminiumoxid ist ein Isolator.
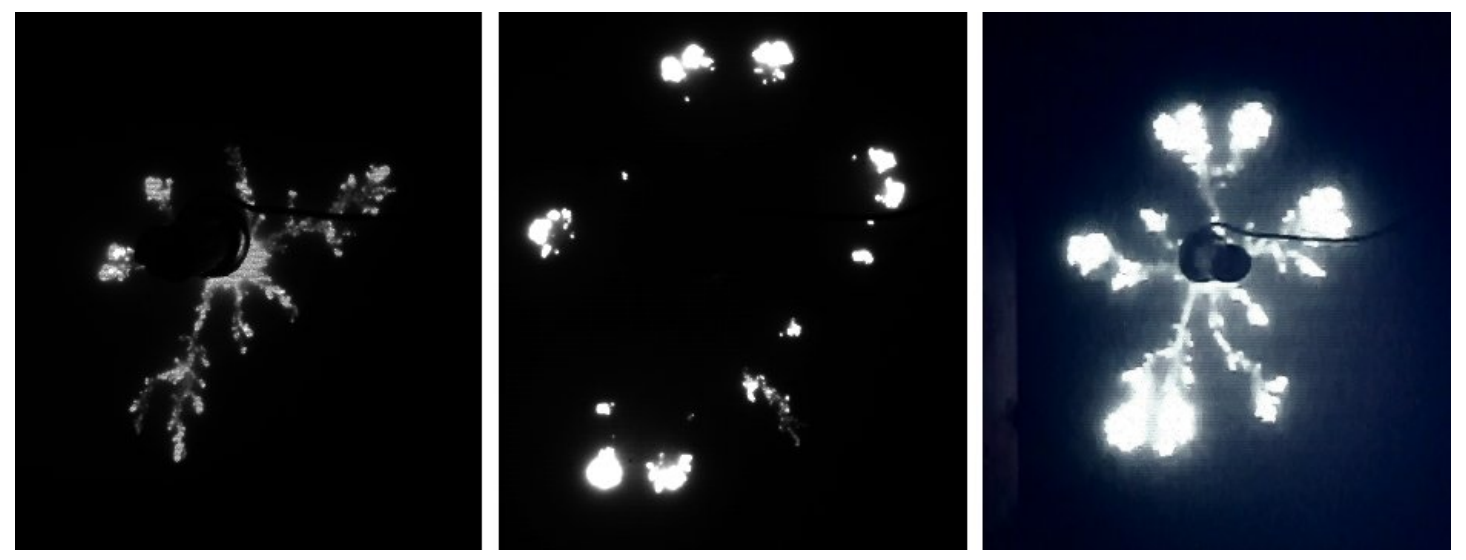

Abbildung 5.25: Brennflecken isolierend beschichtete Wolframkathode; links: Typ II; mitte: Typ I; rechts: gesamte Entladung als Übersicht aus Kamera 2 (101223cine03 - W Al2 ${ }_{2} \mathrm{O}_{3} \mathrm{I}_{\text {Bogen }}=500$ $\left.A, t_{\text {ent }}=500 \mu \mathrm{s}, B_{\text {ext }}=0 \mathrm{mT}, t_{\text {interval }}=58 \mu \mathrm{s} \exp =55 \mu \mathrm{s}\right)$

Auch auf diesen Proben sind zwei unterschiedliche Brennflecktypen zu beobachten. Sie sind in Abbildung 5.25 dargestellt. Links in der Abbildung ist der zweite Typ zu erkennen, er bewegt sich schnell und leuchtet schwach. Der erste Typ hingegen leuchtet stark, bewegt sich langsam und ist in der Mitte der Abbildung zu erkennen. Beide Aufnahmen sind aus der gleichen Entladung (101223cine03 fünfte Entladung). Rechts in der Abbildung ist die gesamte Entladung mit Kamera 2 fotografiert, beide Typen sind darin wieder zuerkennen.

Die von den Brennflecken zurückgelegte Strecke wächst mit jeder Entladung, wie Abbildung 5.26 zeigt. Die Brennflecken vom ersten Typ treten jeweils in der Region auf, in der die Entladung bisher nicht gewesen ist. Nach der Entladung unterscheiden sich die Regionen farblich voneinander. Es entsteht der Eindruck als würde der Brennfleck vom ersten Typ die isolierende Schicht verdampfen, während Typ II auf den bereits frei geräumten Spuren brennt und dort dem Verhalten von Wolfram ähnelt. Die Versuche zur Brennfleckgeschwindigkeit auf Wolfram und beschichtetem Wolfram zeigen, dass die Geschwindigkeit der Brennflecken auf den Spuren in etwa denen von Wolfram entsprechen (siehe Kapitel 
5.2 .2 auf Seite 51.

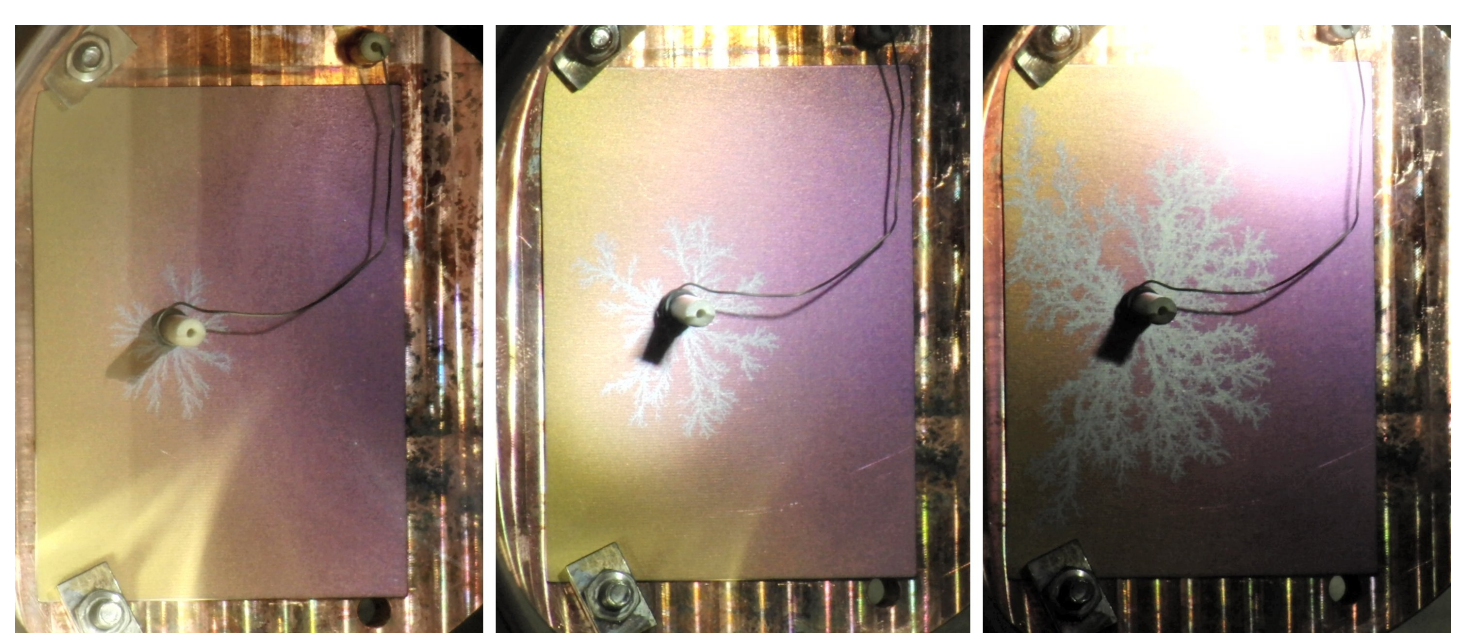

Abbildung 5.26: Isolierend beschichtete Wolfram Kathode, v.l.n.r nach zwei, fünf und 20 Entladungen (101223 - $\mathrm{W} \mathrm{Al2}{ }_{2} \mathrm{O}_{3} I_{\text {Bogen }}=500 \mathrm{~A}, t_{\text {ent }}=500 \mu \mathrm{s}, B_{\text {ext }}=0 \mathrm{mT}$ )

\subsection{Detaillierte Auswertung}

\subsubsection{Strukturebenen der Bogenfußpunkte}

Alle durchgeführten Experimente und Beobachtungen weisen auf drei unterschiedliche Erscheinungsebenen der Bogenfußpunkte hin.

- Bogenspuren

- Brennfleckfronten

- Brennflecken

Alle drei sind unterschiedliche Formen desselben Phänomens. Dessen Erscheinung hängt von der Art und Weise der Beobachtung ab und hat erheblichen Einfluss auf die folgenden Analysen. Die Identifizierung der drei Erscheinungsebenen ist Inhalt dieses Abschnittes.

\section{Bogenspuren}

Die oberste der drei Erscheinungsebenen ist die Form der Bogenspur. Sie ist die oberste, weil sie ohne spezielle technische Hilfsmittel wie zum Beispiel einer Hochgschwindigkeitskamera beobachtet werden kann.

Abbildung 5.27 zeigt eine Entladung auf Wolfram (110126cine02). Es sind sechs Bogenspuren zu erkennen, die sich radial vom Zündstifft entfernen. Die Abstände zwischen den Bogenspuren sind in etwa gleich groß, sie verteilen sich gleichmäßig über die Probenoberfläche.

Jede Bogenspur trägt einen Teil des gesamten Entladungstromes (500 Ampere über 500 $\mu s)$, in diesem Fall 80 Ampere pro Bogenspur. 


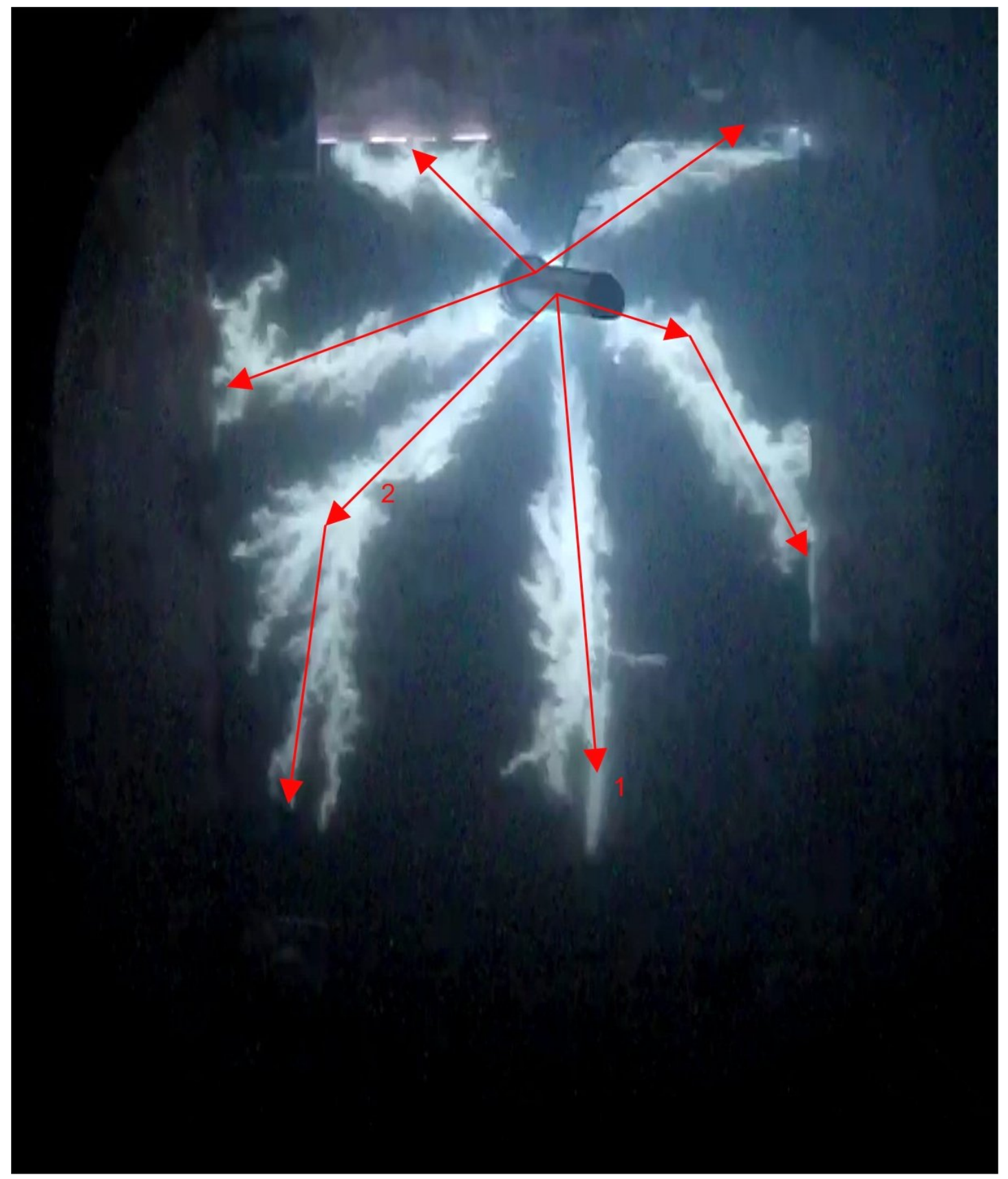

Abbildung 5.27: Sechs Bogenspuren, Entladung auf massiven Wolfram bei 500 Ampere über 500 $\mu s$, bei $B_{\text {ext }}=0 m T$ (110126cine02 - W)

- Bogenspur, Def.: Die Gesamtheit aller zeitlich und räumlich direkt aufeinander folgenden Leuchterscheinungen wird als Bogenspur bezeichnet.

Die Aufnahmen der Bogenspuren wurden mit Kamera 2 gemacht. Abbildung 5.28 bis 5.31 zeigen Bogenspuren beider Typen auf verschieden Materialien. 

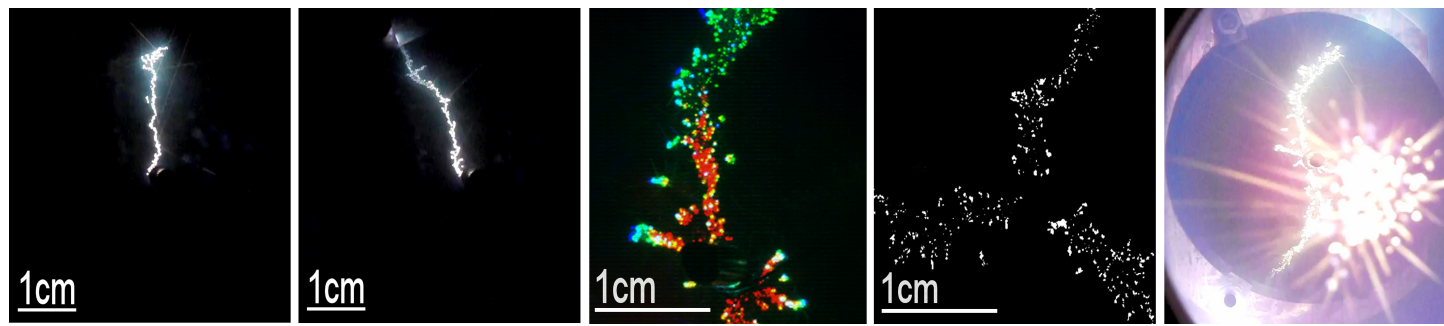

Abbildung 5.28: Bogenspuren auf Kohlenstoffkathoden; magnetische Flussdichte des externen Magnetfeldes (v.l.n.r): $100 \mathrm{mT}, 100 \mathrm{mT}, 100 \mathrm{mT}, 0 \mathrm{mT}, 0 \mathrm{mT}$; Bogenstrom (v.l.n.r): 0,5 kA, 0,5 $k A, 0,5 \mathrm{kA}$, $3 \mathrm{kA}$, $3 \mathrm{kA}$; Zündstifft Durchmesser $3 \mathrm{~mm}$
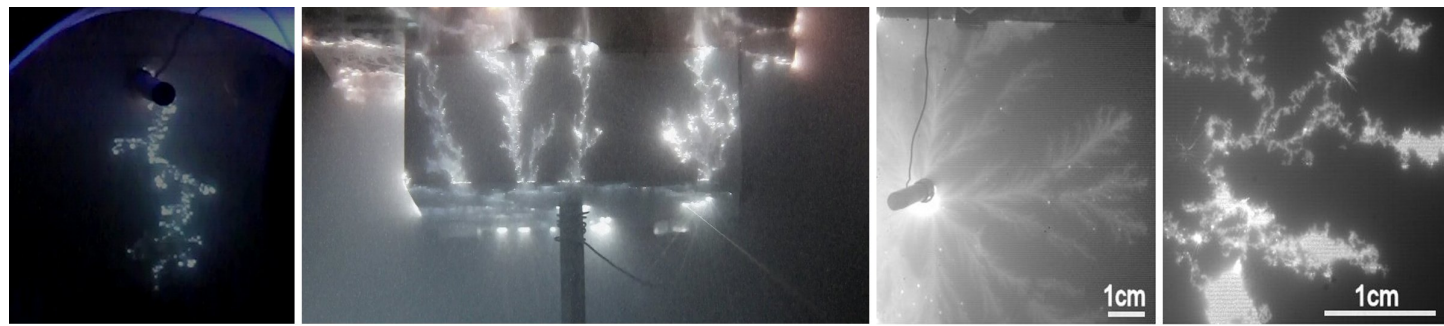

Abbildung 5.29: Bogenspuren auf Wolfram beschichtetem Kohlenstoff; magnetische Flussdichte des externen Magnetfeldes (v.l.n.r): 6,2 mT, $0 \mathrm{mT}, 0 \mathrm{mT}, 0 \mathrm{mT}, 0 \mathrm{mT}$; alle Entladungen 500 Ampere über $500 \mu s$; Zündstifft Durchmesser $3 \mathrm{~mm}$


Abbildung 5.30: Bogenspuren auf Wolframkathoden; magnetische Flussdichte des externen Magnetfeldes (v.l.n.r): $120 \mathrm{mT}, 120 \mathrm{mT}, 0 \mathrm{mT}, 5,6 \mathrm{mT}, 5,6 \mathrm{mT}$; alle Entladungen 500 Ampere über $500 \mu \mathrm{s}$; Zündstifft Duchrmesser $3 \mathrm{~mm}$
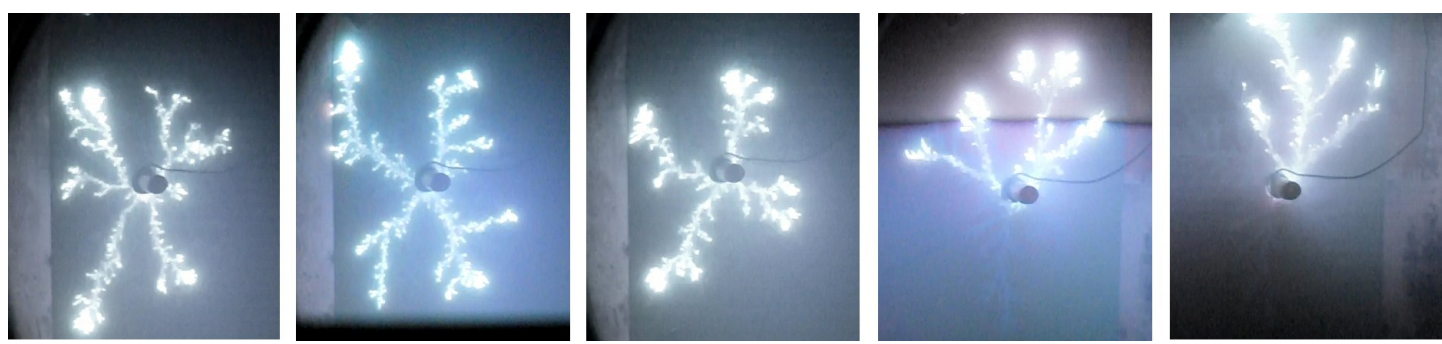

Abbildung 5.31: Bogenspuren auf $\mathrm{Al}_{2} \mathrm{O}_{3}$ beschichtetem Wolfram; magnetische Flussdichte des externen Magnetfeldes (v.l.n.r): $0 \mathrm{mT}, 0 \mathrm{mT}, 0 \mathrm{mT}, 5,6 \mathrm{mT}, 11,7 \mathrm{mT}$; alle Entladungen 500 Ampere über $500 \mu \mathrm{s}$; Zündstifft Durchmesser $3 \mathrm{~mm}$ 


\section{Brennfleckfront (BFF)}

Auf der nächst tieferen Erscheinungsebene der Bogenfußpunkte wird die Form der Brennfleckfronten (BFF) oder Spotfronten sichtbar (in der Literatur teilweise „Grouped Catho-

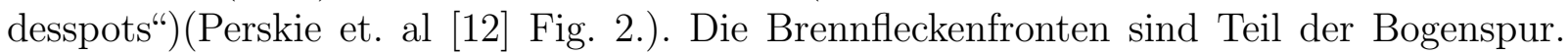
Durch die kurze Belichtungszeit der Hochgeschwindigkeitskamera (wenige Mikrosekunden) wird die Bogenspur in einzelne Abschnitte, geteilt. Diese Abschnitte lassen sich bei hoher Belichtungszeit der Fotos wieder zu einer Bogenspur zusammensetzen.

Abbildung 5.32 zeigt BFFs einer Entladung auf Wolfram (110126cine02, ebenfalls Abbildung 5.27). Für den linken Teil der Darstellung in Abbildung 5.32 wird nur jedes zweite Bild genutzt. Die BFF's der ersten Bogenspur sind gelb und die der zweiten Bogenspur blau markiert. Einige der markierten $\mathrm{BFF}^{\prime} \mathrm{s}$ wurden vom linken in den rechten Teil der Abbildung übertragen.

Zusätzlich ist im rechten Teil der Abbildung die Summe aller einzelnen BFF's der Aufnahme dargestellt, diese Darstellung zeichnet zwei der Bogenspuren aus Abbildung 5.27 nach $\left(t_{\text {interval }}=21 \mu s \exp =2 \mu s\right)$.
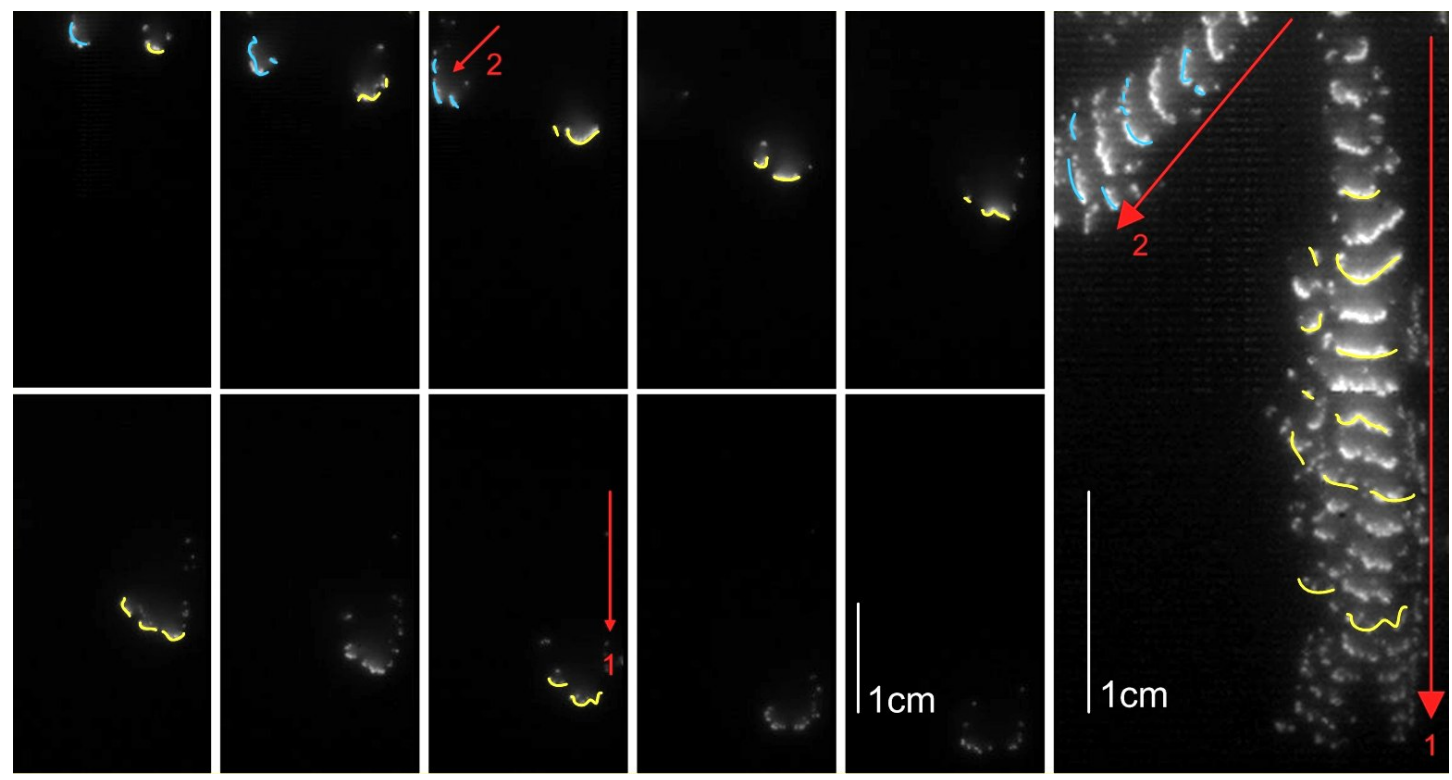

Abbildung 5.32: Brennfleckfronten einer Entladung auf massiven Wolfram bei 500 Ampere über $500 \mu s$ (110126cine02), Links: BFF's einzeln, gelb und blau markiert (Bildabstand 42 $\mu s$ bei 2 $\mu s$ exp); rechts: alle einzelnen BFF's kumuliert (Bildabstand $21 \mu s$ bei $2 \mu s$ exp)

- Brennfleckfront, Def.: Der innerhalb der Belichtungszeit der Hochgeschwindigkeitskamera aufgenommene Teil einer einzelnen Bogenspur wird als Brennfleckfront bezeichnet.

Bei der anschließenden Geschwindigkeitsuntersuchung muss auf die Zuordnungsfähigkeit der BFF's geachtet werden. Einer Brennfleckfront muss ein direkter Vorgänger oder Nachfolger zugeordnet werden können. So zum Beispiel in Abbildung 5.32. Die Geschwindigkeit der gelben Brennflecken untereinander kann bestimmt werden, nicht jedoch die Geschwindigkeit von blauen zu gelben Brennfleckfronten. Deshalb wurden die Geschwindigkeitsuntersuchungen auf die Brennfleckfronten der einzelnen Bogenspuren bezogen. 


\section{Brennflecken}

In der untersten der drei Erscheinungsebenen erhalten die Bogenfußpunkte die Form der Brennflecken oder Kathodenspots. Aus einer Vielzahl von Brennflecken setzen sich die Brennfleckfronten bzw. die Bogenspuren zusammen. Sie sind nicht direkt darstellbar, da alle Leuchterscheinungen auf das Plasma über den Brennflecken zurück zu führen sind und die Brennflecken schon deshalb nicht dargestellt werden können. Ihre Existenz lässt sich auf die Krater an der Kathodenoberfläche zurückführen. Problematisch ist hierbei, dass keine Krater von Subspots des ersten Typ's beobachtet wurden.

Die Krater wurden in Abbildung 5.33 rechts mit dem REM fotografiert, der Kraterdurchmesser liegt zwischen $1 \mu \mathrm{m}$ und $10 \mu \mathrm{m}$. Die Annahmen ist, dass jeder Krater einem Brennfleck des zweiten Typ's entspricht.

Aufnahme 5.33 links zeigt die Leuchterscheinung eines oder mehrerer Brennflecken von 1,2 mm größe. Dem Maßstab beider Aufnahmen ist zu entnehmen, dass zwischen Leuchterscheinung und Brennfleck ca. drei Größenordnungen liegen.


Abbildung 5.33: Links: Krater von einigen Mikrometern auf erodierter CW - Oberfläche; rechts: Brennfleck bei einer $\mu$ s exp.

- Brennfleck, Def.: Die elementarste Form der Bogenfußpunkte wird als Brennfleck bezeichnet.

Die Aufnahmen 5.34 wurden mit Kamera 1 der Hochgeschwindigkeitskamera gemacht. Sie stellt den Grenzbereich der Versuchsanordnung dar, kleinere Strukturen und kürzere Schritte könne nicht aufgelöst werden. 

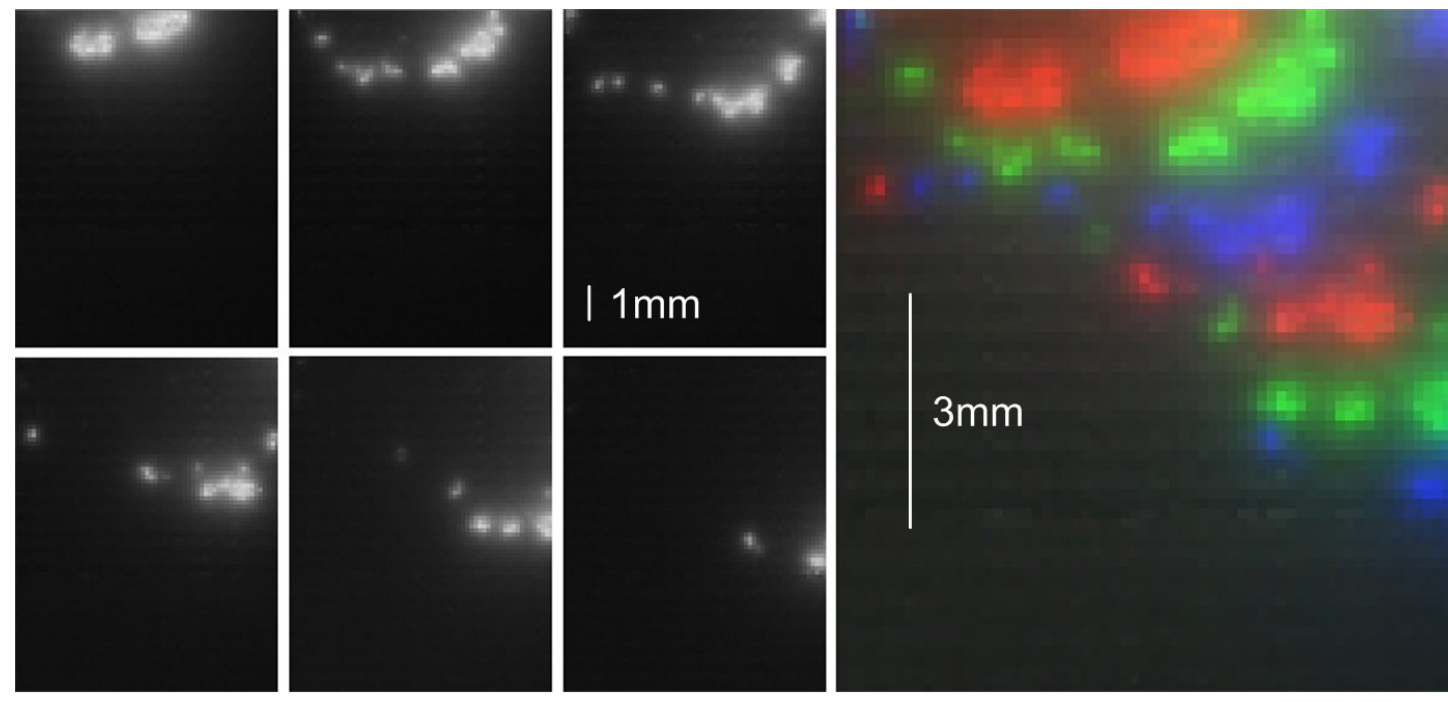

Abbildung 5.34: Links: Brennflecken bzw. Brennfleckfronten einer Entladung auf Wolfram beschichtetem Kohlenstoff bei 500 Ampere Bogenstrom über $500 \mu$ s ohne externes Magnetfeld; Bildabstand $8 \mu \mathrm{s}$, $\exp 1 \mu \mathrm{s}$; erste Reihe (v.l.n.r) rot, grün, blau, zweite Reihe (v.l.n.r) rot, grün, blau; rechts: $R G B$ kumuliert

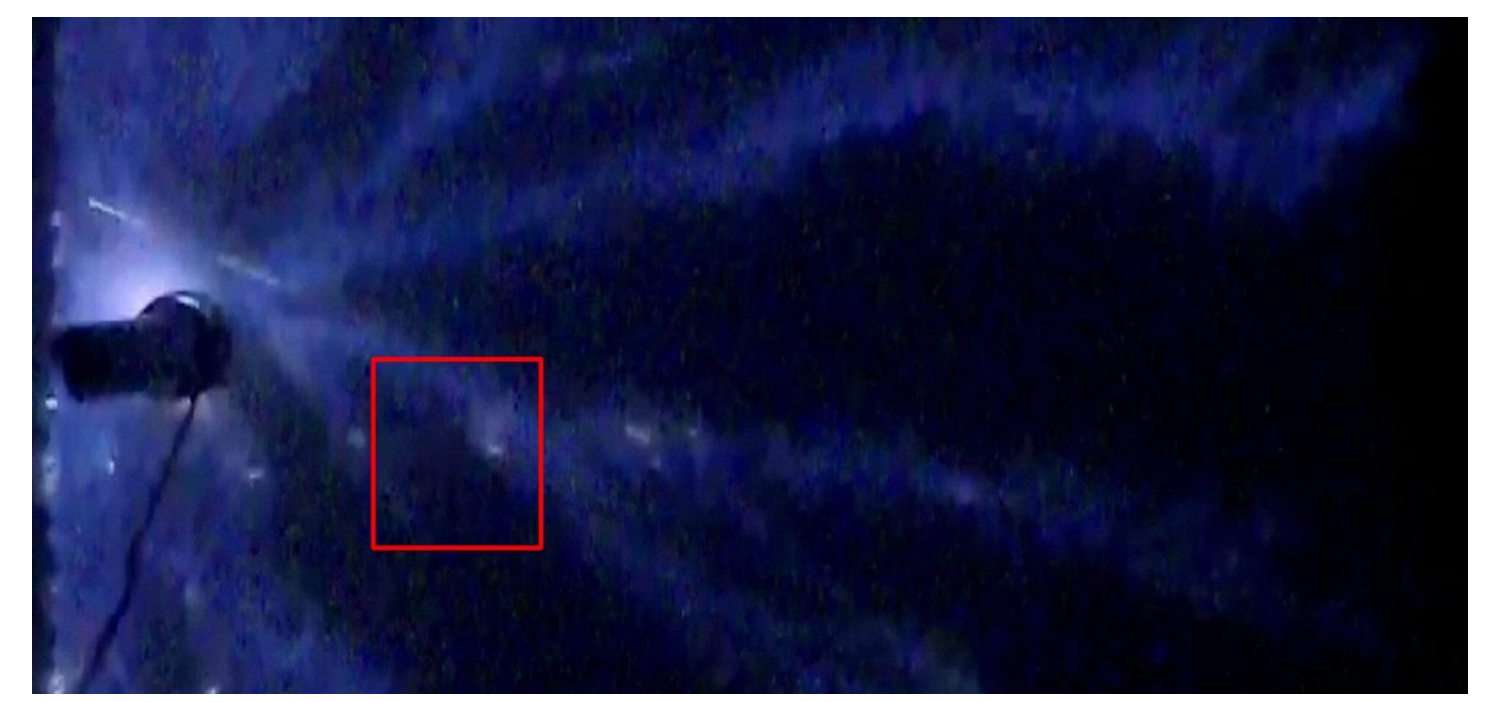

Abbildung 5.35: Übersichtsaufnahmen von Entladung 110119 CW Wafer N500 cine02 (Abbildung 5.34)

\subsubsection{Geschwindigkeiten der Brennfleckenfront}

Der in Kapitel 4.2.1 beschriebene Versuchsaufbau und die zwei in Kapitel 3.2.1 dargelegten Messmethoden zur Geschwindigkeitsanalyse der Brennfleckfrontbewegung führten zu folgenden, nach dem Kathodenmaterial gegliederten, Ergebnissen. Alle Entladungen wurden unter Hoch- bis Ultrahochvakuum Bedingungen, und sofern nicht anders angegeben, mit 500 Ampere Bogenstrom gezündet. Die Werte der magnetischen Flussdichte ergeben sich aus deren Messung und der Extrapolation der Messwerte für geringen Bogenstrom. 


\section{Kohlenstoff - C}

Die Lebenszeit der Brennflecken auf Kohlenstoff ist deutlich länger als die der Brennflecken auf anderen Kathodenmaterialien. Wegen der daraus resultierenden geringen Geschwindigkeit der Brennfleckfront, konnte deren Abhängigkeit von der Größe der magnetischen Flussdichte des externen Magnetfeldes für Kohlenstoff bestimmt werden. Für alle anderen Materialien sind die Geschwindigkeiten bei höheren Feldstärken bzw. Flussdichten so groß, dass die Beobachtung der Brennflecken in der Kreuzflanschkammer nur teilweise möglich ist. Auf den Kohlenstoffkathoden konnten die zwei Brennflecktypen nicht unterschieden werden. Um den genannten Zusammenhang darzustellen, werden die Geschwindigkeiten der Brennfleckfront über der magnetischen Flussdichte aufgetragen, wie in Abbildung 5.36 dargestellt ist.

In Tabelle 5.1 sind die Durchschnittsgeschwindigkeiten der in Grafik 5.36 abgebildeten Messungen und deren Standardabweichung dargestellt. Der Einfluss der magnetischen Flussdichte auf die Brennfleckgeschwindigkeit, im Bereich von fünf bis $150 \mathrm{mT}$, ist linear. Die Ausgleichsgerade in diesem Bereich ergibt einen Proportionalitätsfaktor von $m=\frac{\Delta y}{\Delta x}=2,42 m T / m^{-1}$.



Abbildung 5.36: Geschwindigkeit der Brennfleckfront über der magnetischen Flussdichte für Kohlenstoff, grafisch; Messwert und Ausgleichsgerade 
Tabelle 5.1: Durchschnittliche Geschwindigkeit der Brennfleckfront und die magnetische Flussdichte bei Kohlenstoff, tabellarisch

\begin{tabular}{cccccc}
\hline $\mathrm{B}$ in $\mathrm{mT} \pm 4 \%$ & 0 & 5,6 & 11,2 & 20,3 & 29,5 \\
$\mathrm{v}$ in $\mathrm{m} / \mathrm{s} \pm 1 \%$ & 6,7 & 11,1 & 20,8 & 23,6 & 25,3 \\
$\sigma$ in $\mathrm{m} / \mathrm{s}$ & 2,3 & 7,7 & 11,0 & 11,7 & 8,7 \\
\hline & & & & & \\
\hline $\mathrm{B}$ in $\mathrm{mT} \pm 4 \%$ & 38,8 & 48,0 & 57,7 & 67,5 & 77,6 \\
$\mathrm{v}$ in $\mathrm{m} / \mathrm{s} \pm 1 \%$ & 34 & 30 & 40,2 & 44,2 & 44,2 \\
$\sigma$ in $\mathrm{m} / \mathrm{s}$ & 10,4 & 14,6 & 12,3 & 4,4 & 8,5 \\
\hline & & & & & \\
\hline $\mathrm{B}$ in $\mathrm{mT} \pm 4 \%$ & 87,6 & 109,3 & 129,5 & 143,3 & \\
$\mathrm{v}$ in $\mathrm{m} / \mathrm{s} \pm 1 \%$ & 50,6 & 42,3 & 71,4 & 62,4 & \\
$\sigma$ in $\mathrm{m} / \mathrm{s}$ & 18,6 & 10,8 & 21,6 & 18,2 & \\
\hline
\end{tabular}

Frühere Versuche zeigten, dass die Geschwindigkeit nur bis zu einer materialspezifischen magnetischen Flussdichte linear ansteigt, danach wird der Geschwindigkeitsanstieg immer geringer. Für Titan, Molybdän und Edelstahl wurde der lineare Bereich bis $500 \mathrm{mT}$ nach gewiesen, wobei der Proportionalitätsfaktor $8 \mathrm{mT} / \mathrm{ms}^{-1}$ beträgt [19]. Für Kupferentladungen von 450 A konnte ein linearer Anstieg der Geschwindigkeits von $4 \mathrm{mT} / \mathrm{ms}^{-1}$ bis zu 200 bis $300 \mathrm{mT}$ nachgewiesen werden 12

Grafik 5.36 und Tabelle 5.1 zeigen, dass sich die Brennflecken auch ohne ein externes Magnetfeld bewegen. Die Bewegung wird, wie in Kapitel 2.6 auf Seite 9 beschrieben, auf das interne Magnetfeld der Brennflecken zurück geführt.

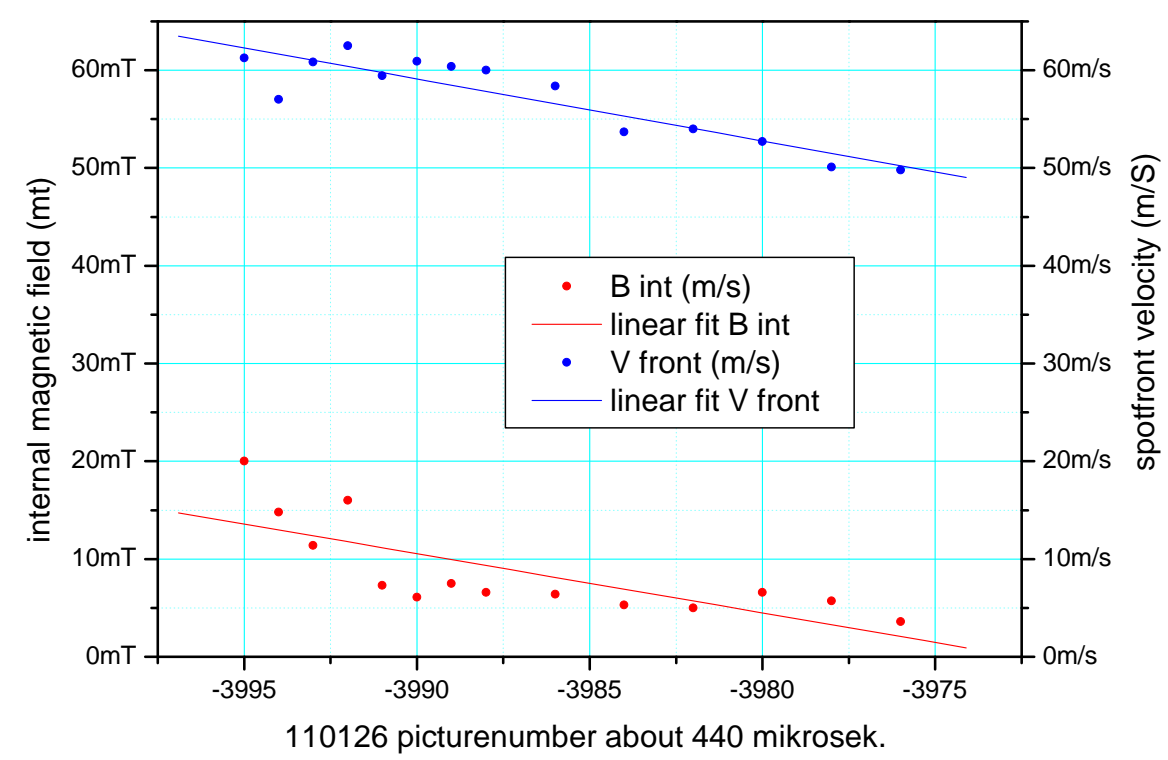

Abbildung 5.37: Internes Magntefeld Bint (rot) und Geschwindigkeit der BFF (blau) über der Entladungsdauer (Bildnummern) 
Mit größerer Entfernung vom Zündstift und somit größerem Abstand der Bogenspuren zueinander, wird auch die Wechselwirkung der einzelnen Bogenspuren aufgrund ihrer internen Magnetfelder geringer, weshalb der Radius der BFF größer wird (die Bogenspuren werden dicker). Nach Perskii ergibt sich das interne Magnetfeld der Brennflecken bzw. deren Front $\mathrm{zu} B_{\text {int }}=\frac{\mu_{0}}{4 \pi} \cdot \frac{I_{B o g e n}}{R_{B F F}} \sqrt{12}$. Für Entladung $110126-\mathrm{W}$ wurde der Radius der BFF gemessen und entsprechend der Bogenspur (80 A) das interne Magnetfeld berechnet. Zusätzlich wurde die Geschwindigkeit der BFF gemessen und ebenfalls in Abbildung dargestellt 5.37 . Sherman et al. stellten das Steigen der Brennfleckgeschwindigkeit aufgrund des internen Magnetfeldes der Brennflecken für Kupfer bis zu 700 mT dar ( 15] Figure. 3).

Ein erhöhter Bogenstrom, das heißt höhere magnetische Flussdichten des internen magnetischen Feldes führen zu einer erhöhten Brennfleckgeschwindigkeit bzw. zu einer schlankeren Bogenspur. Der Einfluss des Bogenstromes auf die Geschwindigkeit der Brennfleckfront auf Kohlenstoffkathoden ist in Tabelle 5.2 dargestellt.

Tabelle 5.2: Bogenstrom und Geschwindigkeit der Brennfleckfront auf Kohlenstoffkathoden

\begin{tabular}{cccc}
\hline$I_{\text {bogen }}$ in $\mathrm{kA} \pm 4 \%$ & 0,5 & 1 & 3 \\
$\mathrm{v}$ in $\mathrm{m} / \mathrm{s} \pm 1 \%$ & $6,4 \mathrm{~m} / \mathrm{s}$ & $5,1 \mathrm{~m} / \mathrm{s}$ & $40,0 \mathrm{~m} / \mathrm{s}$ \\
$\sigma$ in $\mathrm{m} / \mathrm{s}$ & 2,0 & 1,7 & 15,3 \\
\hline
\end{tabular}

\section{Wolfram - W}

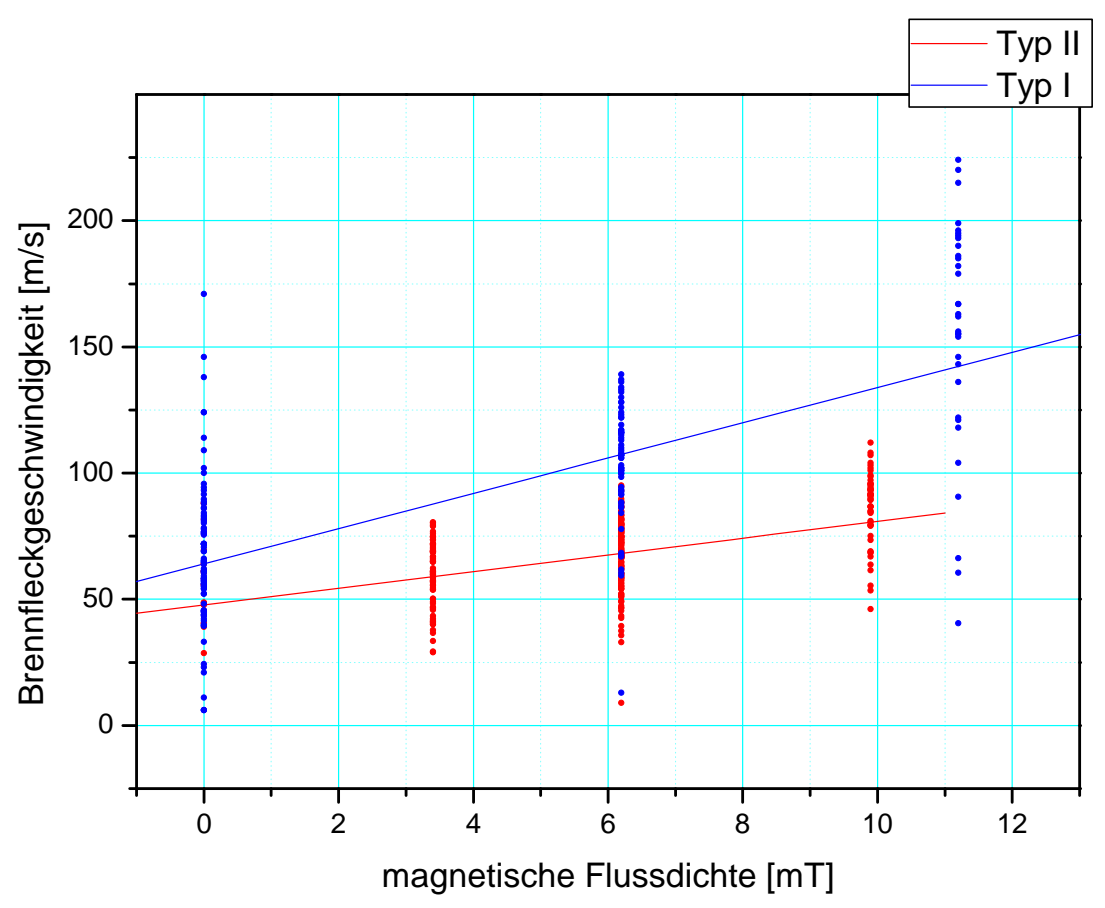

Abbildung 5.38: Geschwindigkeit der Brennfleckfront über der magnetischen Flussdichte für Wolframkathoden für Typ I und Typ II, grafisch; Messwert und Ausgleichsgerade 
Auf den Wolframkathoden wie auch auf den weiteren Materialien konnten die Brennflecktypen unterschieden werden. Gegenstand der Untersuchung sind dementsprechend die Geschwindigkeit der Fronten beider Brennflecktypen und deren Abhängigkeit von der magnetischen Flussdichte bei geringer Feldstärke. Tabelle 5.3 und Abbildung 5.38 stellen die Ergebnisse dar. Offenbar werden Brennflecken vom zweiten Typ stärker von einem externen Magnetfeld beeinflusst als Brennflecken des ersten Typs. Ohne ein solches Feld unterscheiden sich die beiden Brennflecktypen in ihrer Geschwindigkeit nur wenig.

Tabelle 5.3: Durchschnittliche Geschwindigkeit der Brennfleckfront und die magnetische Flussdichte bei Wolfram für Typ I und Typ II, tabellarisch

\begin{tabular}{|c|c|c|c|c|c|}
\hline \multicolumn{6}{|l|}{ Typ I } \\
\hline $\mathrm{B}$ in $\mathrm{mT} \pm 4 \%$ & 0 & 3,4 & 6,2 & 9,9 & \\
\hline $\mathrm{v}$ in $\mathrm{m} / \mathrm{s} \pm 1 \%$ & 57,9 & 59,2 & 68,1 & 87,1 & \\
\hline$\sigma$ in $\mathrm{m} / \mathrm{s}$ & 16,2 & 13,3 & 13,5 & 14,6 & \\
\hline \multicolumn{6}{|l|}{ Typ II } \\
\hline $\mathrm{B}$ in $\mathrm{mT} \pm 4 \%$ & 0 & & 6,2 & & 11,2 \\
\hline $\mathrm{v}$ in $\mathrm{m} / \mathrm{s} \pm 1 \%$ & 66,7 & & 104,1 & & 157,8 \\
\hline$\sigma$ in $\mathrm{m} / \mathrm{s}$ & 29,1 & & 23,0 & & 47,1 \\
\hline
\end{tabular}

\section{Wolfram Kohlenstoff - CW}

Auf Wolfram beschichtetem Kohlenstoff unterscheiden sich die beiden Brennflecktypen hinsichtlich der Geschwindigkeit ihrer Front stark voneinander. Die Brennflecken vom ersten Typ sind deutlich schneller, im externem Magnetfeld sowie außerhalb. Brennflecktyp eins wird vom Magnetfeld sehr viel stärker beeinflusst. Die Ergebnisse der Messungen sind in Abbildung 5.39 Tabelle und Tabelle 5.4 dargestellt. 


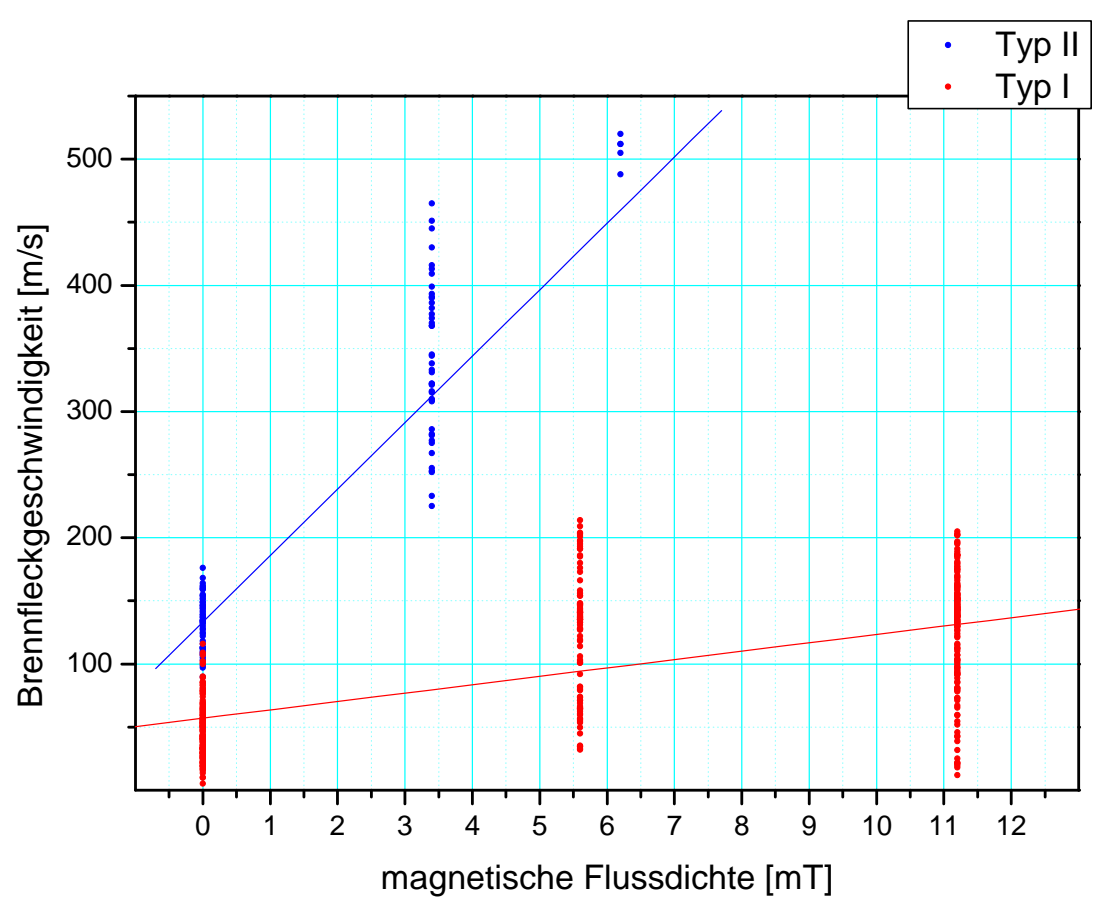

Abbildung 5.39: Geschwindigkeit der Brennfleckfront über der magnetischen Flussdichte für Wolfram beschichtete Kohlenstoffkathoden für Typ I und Typ II, grafisch; Messwert und Ausgleichsgerade

\begin{tabular}{cccc}
\hline Typ II & & & \\
\hline $\mathrm{B}$ in $\mathrm{mT} \pm 4 \%$ & 0 & 3,4 & 6,2 \\
$\mathrm{v}$ in $\mathrm{m} / \mathrm{s} \pm 1 \%$ & 133,7 & 342,7 & 507,4 \\
$\sigma$ in $\mathrm{m} / \mathrm{s}$ & 20,6 & 62,4 & 12,07 \\
\hline & & & \\
& & & \\
\hline Typ I & & & \\
\hline $\mathrm{B}$ in $\mathrm{mT} \pm 4 \%$ & 0 & 5,6 & 11,2 \\
$\mathrm{v}$ in $\mathrm{m} / \mathrm{s} \pm 1 \%$ & 48,5 & 123,2 & 129,3 \\
$\sigma$ in $\mathrm{m} / \mathrm{s}$ & 22,9 & 50,4 & 44,4 \\
\hline
\end{tabular}

Tabelle 5.4: Durchschnittliche Geschwindigkeit der Brennfleckfront und die magnetische Flussdichte bei Wolfram beschichtete Kohlenstoffkathoden für Typ I und Typ II, tabellarisch

\section{Aluminiumoxid Wolfram - Walo}

Im Versuch zeigte sich, dass sich die auf der Schicht brennenden Brennflecken um ein vielfaches langsamer bewegen und nur wenig vom externen Magnetfeld beschleunigt werden können. Auf der von den Vorgängern hinterlassenen Spur hingegen haben die Brennflecken eine ähnliche Geschwindigkeit wie auf unbeschichtetem Wolfram (Siehe Kapitel 5.1.4: Beobachtungen auf beschichtetem Wolfram, auf Seite 45). Die Ergebnisse der Messungen sind sowohl grafisch in Abbildung 5.40, als auch tabellarisch in Tabelle 5.5 dargestellt. 


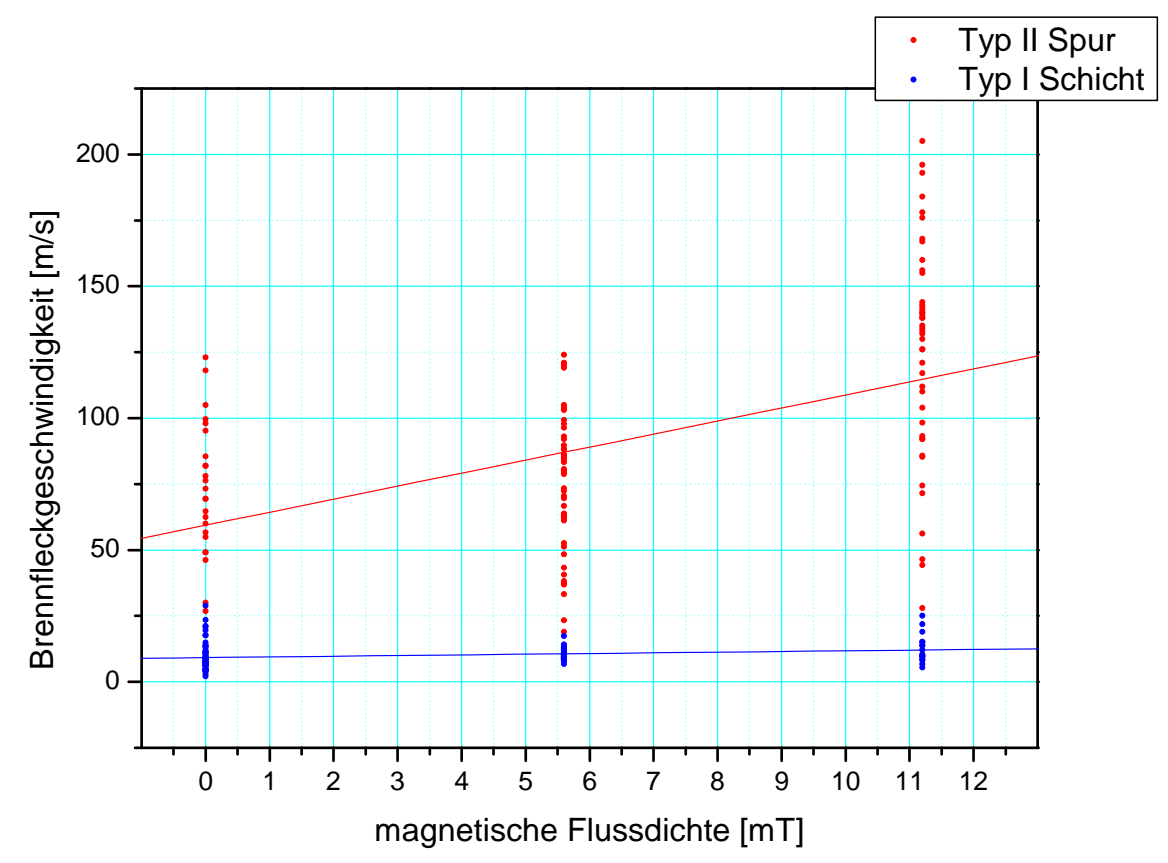

Abbildung 5.40: Geschwindigkeit der Brennfleckfront über der magnetischen Flussdichte für Kathoden aus $\mathrm{Al}_{2} \mathrm{O}_{3}$ beschichtetem Wolfram für Typ I und Typ II, grafisch; Messwert und Ausgleichsgerade

\begin{tabular}{cccc}
\hline Spur (Typ II) & & & \\
\hline $\mathrm{B}$ in $\mathrm{mT} \pm 4 \%$ & 0 & 5,6 & 11,1 \\
$\mathrm{v}$ in $\mathrm{m} / \mathrm{s} \pm 1 \%$ & 73,1 & 75,6 & 126,2 \\
$\sigma$ in $\mathrm{m} / \mathrm{s}$ & 25,1 & 25,8 & 41,6 \\
\hline
\end{tabular}

\begin{tabular}{cccc}
\hline Schicht (Typ I) & & & \\
\hline $\mathrm{B}$ in $\mathrm{mT} \pm 4 \%$ & 0 & 5,6 & 11,1 \\
$\mathrm{v}$ in $\mathrm{m} / \mathrm{s} \pm 1 \%$ & 9,3 & 10,3 & 12,7 \\
$\sigma$ in $\mathrm{m} / \mathrm{s}$ & 5,1 & 2,1 & 5,1 \\
\hline
\end{tabular}

Tabelle 5.5: Durchschnittliche Geschwindigkeit der Brennfleckfront und die magnetische Flussdichte bei isolierend beschichtetem Wolfram für Typ I und Typ II, tabellarisch

\section{Fehlerbetrachtung}

Um die Genauigkeit der angegebenen Geschwindigkeiten abzuschätzen, müssen verschieden Faktoren berücksichtigt werden. Dabei spielen systematische und zufällige Fehler, sowie der Vakuumbogenbrennfleck selbst eine unterschiedliche Rolle. Im Folgenden werden die relevanten Fehlerquellen für die magnetische Flussdichte und die Brennfleckgeschwindigkeit genannt, abgeschätzt und bewertet.

Die Inhomogenität und die Messung des magnetischen Feldes gehören zu den systematischen Fehlern. Die Unterschiede der Flussdichte aufgrund der räumlichen Ausdehnung im 
Bereich des Probenhalters betragen bei 500 Ampere Spulenstrom ca. $\pm 2 \%$. Die Qualität der Feldmessung wird vom Hersteller des Messgerätes mit einem Fehler von $\pm 2 \%$ angegeben. Demnach beträgt die Messwertabweichung für die magnetische Flussdichte insgesamt $\pm 4 \%$.

Zu den zufälligen Fehlern wird die Benutzung der zur Auswertung der Bilder genutzten Phantom Camera Control Software v9.2 gezählt. Die Benutzung der Software, dass heißt das Bestimmen der Distanzen durch Mausklick, sowie gerundete Zeitangaben der Software, führt zu einem Fehler der mit $\pm 1 \%$ der gemessenen Geschwindigkeit abgeschätzt werden kann.

Die Fehlerquellen führen zu einer Messwertabweichung, die für die Geschwindigkeitsmessung klein und für die Magnetfeldmessung etwas größer ist. Unabhängig davon muss die große Streuung $(\sigma)$ der Messergebnisse mit dem chaotischen Verhalten des Vakuumbogenbrennfleckes begründet werden. (Siehe Tabelle 5.1, 5.3, 5.4 und 5.5).

\subsubsection{Reaktion der Brennflecken auf schnelle Stromänderungen}

Bei den Beobachtungen der Brennflecken auf C- und CW-Proben konnten, unter anderem, Schwankungen in der Intensität des Leuchtens der Brennflecken festgestellt werden. Die folgende Betrachtung stellt den Bogenstrom der Leuchtintensität der Brennflecken gegenüber.

Der vom Plasma zu übertragende Bogenstrom (Gleichstrom) wird von der Stromquelle bereitgestellt. Die Stromquelle moduliert den Strom ähnlich wie ein Zweipunktregler. Es wird vermutet, dass die hochfrequenten Schwankungen des Bogenstromes den Schwankungen in der Leuchtintensität der Brennflecken entsprechen. Um dies nachzuweisen, wird die Leuchtintensität der Brennflecken sowie der Bogenstrom über die Entladungsdauer aufgetragen.

Die Leuchtintensität der Brennflecken ergibt sich hierbei aus den Bildern der Hochgeschwindigkeitskamera. Nachdem die Bilder ausgewählt und beschnitten worden sind, wird mit der Software Matlab R2009a das Flächenintegral der Intensitäten über jedes einzelne Bild errechnet, als absoluter Wert ausgegeben und anschließend normiert. Der normierte Wert wird dem Zeitpunkt des entsprechenden Bildes im Oszillogramm der Entladung zugeordnet. 


\section{Wolfram Kohlenstoff - CW}

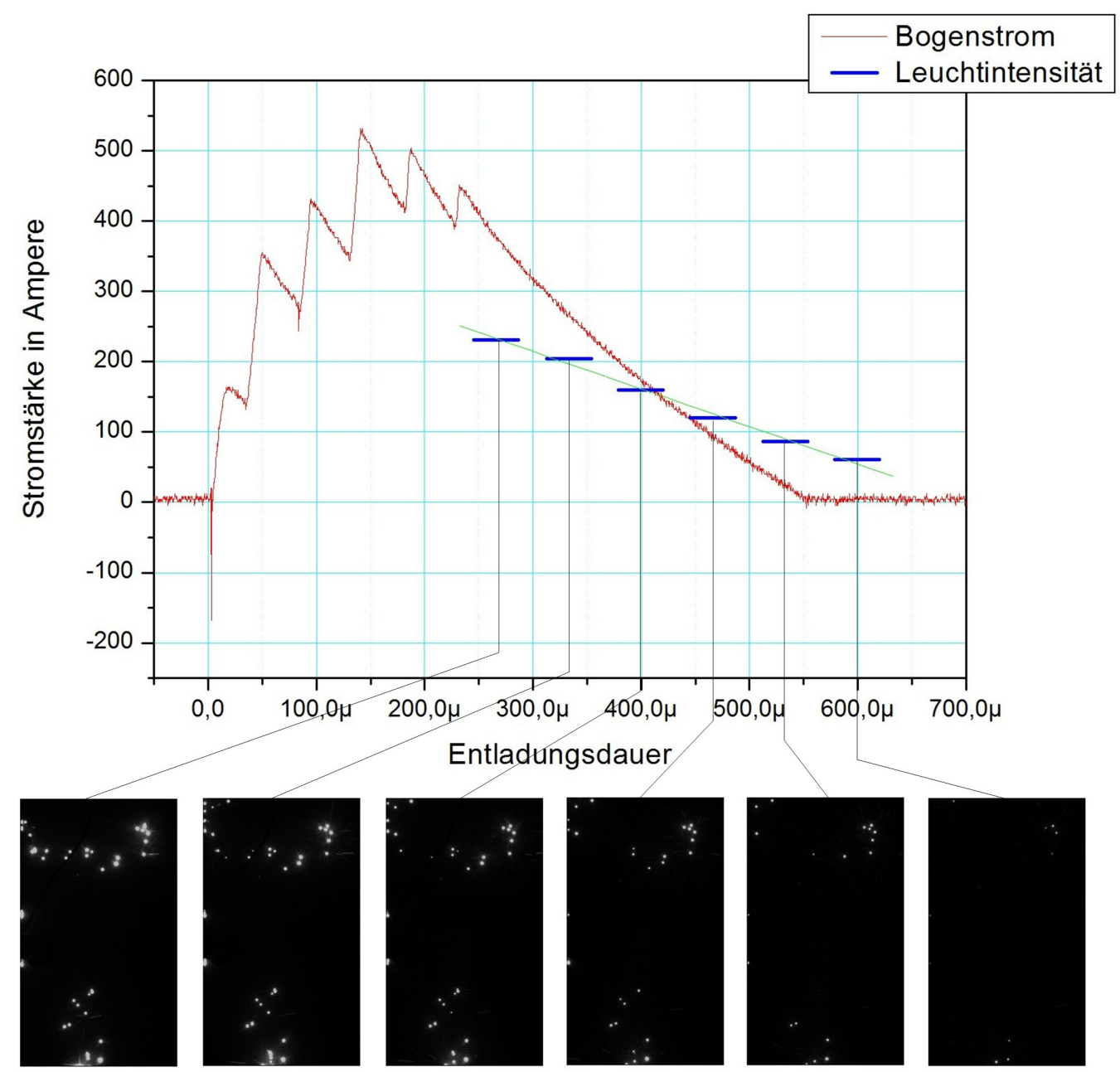

Abbildung 5.41: Oszillogram einer Wolfram beschichteten Kohlenstoff Probe (Bogenstrom - rot) mit relativer Leuchtintensität der Brennflecken (blau); Leuchtstärken sind den Hochgeschwindigkeitbildern zugeordnet

Abbildung 5.41 zeigt das Oszillogramm einer Entladung auf Wolfram beschichtetem Kohlenstoff. 500 Ampere Bogenstrom und $500 \mu s$ Entladungsdauer wurden an der Stromquellensteuerung eingestellt. Während der ersten Hälfte der Entladung (bis ca. $230 \mu s$ ) sind die beschriebenen Stromschwankungen zu erkennen, danach ein kontinuierlicher Abfall der Stromstärke. Die Leuchtintensitäten (blaue Striche) sinken ebenfalls (ab ca. $230 \mu s$ ). Offenbar bestätigt sich die Vermutung eines Zusammenhanges zwischen Bogenstrom und Leuchtstärke der Brennflecken für Kohlenstoffkathoden. Darüber hinaus ist, wie in Kapitel 5.1.1 auf Seite 31 dargestellt, auch in dieser Aufnahmen das Nachglühen der Brennflecken zu erkennen. Im vorderen Bereich der Kurve wurden die Leuchtintensitäten nicht eingetragen, denn dort ist die Belichtungszeit groß gegen die kurzen Stromschwankungen. 


\section{Wolfram - W}

Abbildung 5.43 zeigt den Bogenstrom und die Leuchtintensität über der Entladungsdauer für eine Wolframkathode. Die Bogenparameter betragen 500 Ampere und $500 \mu s$ Entladungsdauer. Einen Zusammenhang von Leuchtintensitäten und Bogenstrom scheint es für Wolframkathoden nicht zu geben.

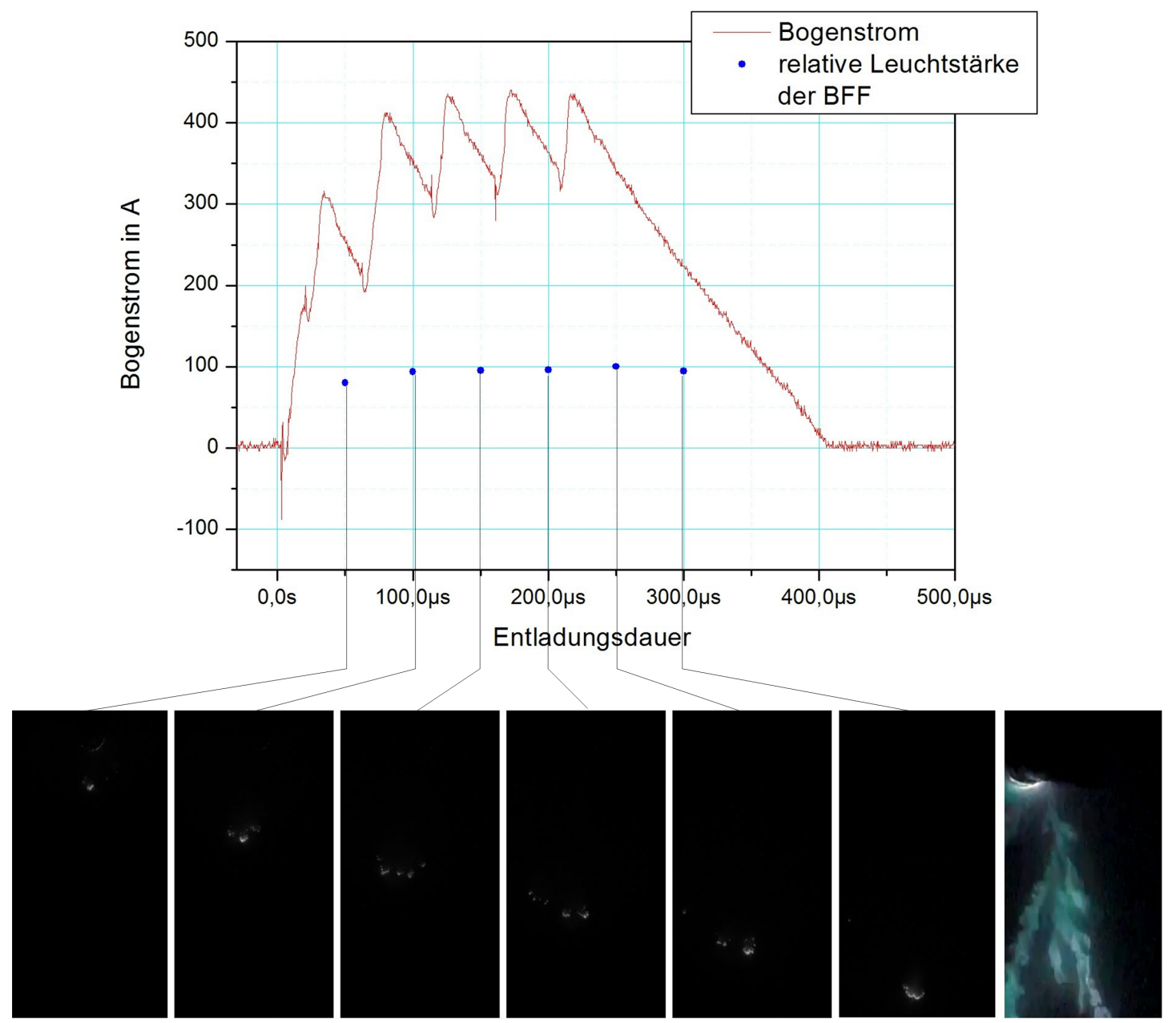

Abbildung 5.42: Oszillogram einer Wolfram Probe (Bogenstrom - rot) mit relativer Leuchtintensität der Brennfleckfronten (blau); Leuchtstärken sind den Hochgeschwindigkeitsbildern zugeordnet, rechts: Übersichtsaufnahme durch Kamera 2

\section{Kohlenstoff}

Abbildung 5.43 zeigt ebenfalls den Bogenstrom und die Leuchtintensität über der Entladungsdauer. Die Bogenparameter betragen 500 Ampere und 2 ms Entladungsdauer. Aufgrund der kurzen Belichtungszeit von einer Mikrosekunde können jetzt die Leuchtintensitäten mit den kurzen Stromschwankungen verglichen werden. 


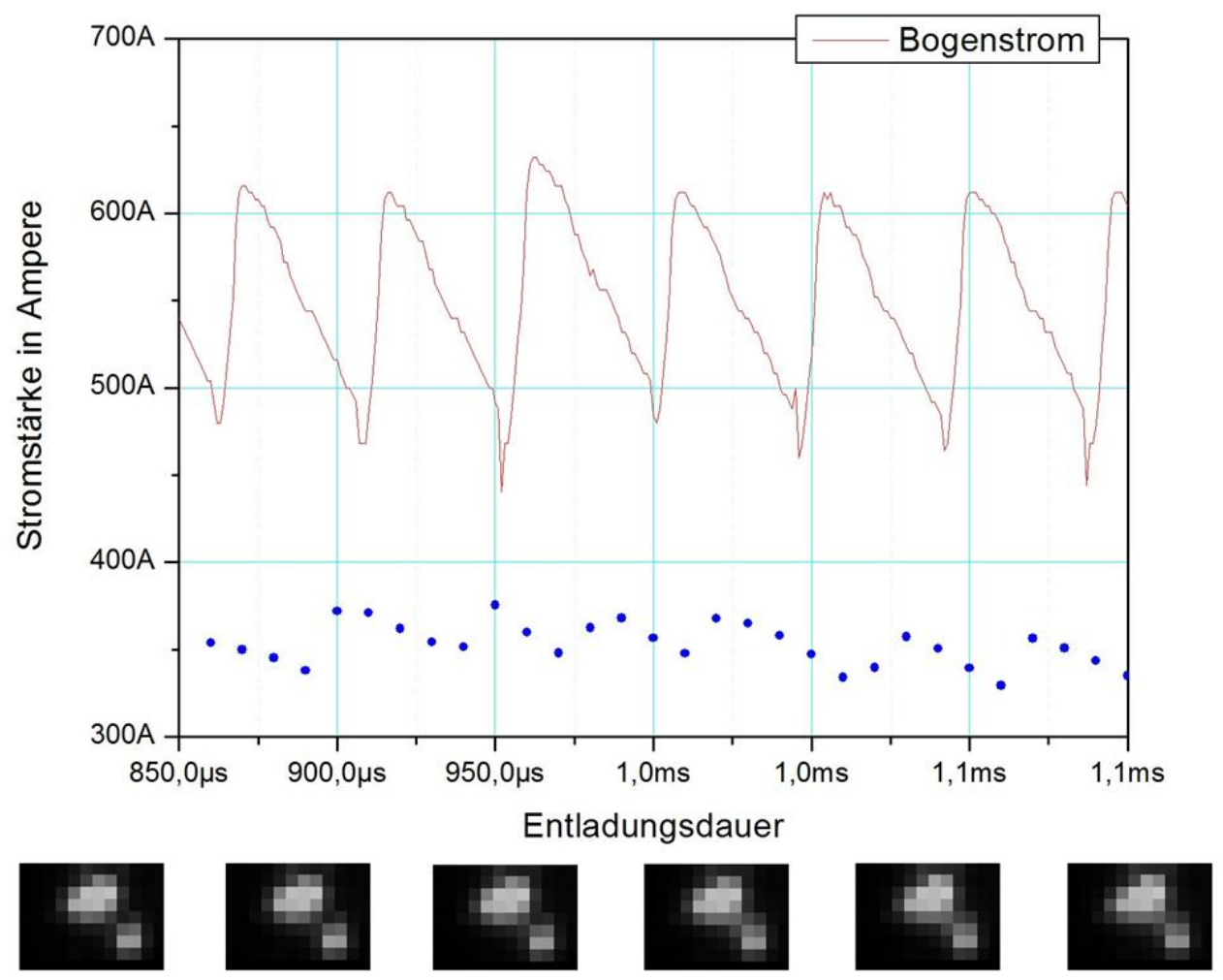

Abbildung 5.43: Oszillogram einer Kohlenstoff Probe (Bogenstrom - rot) mit relativer Leuchtintensität der Brennflecken (blau); Leuchtstärken sind den Hochgeschwindigkeitsbildern zugeordnet

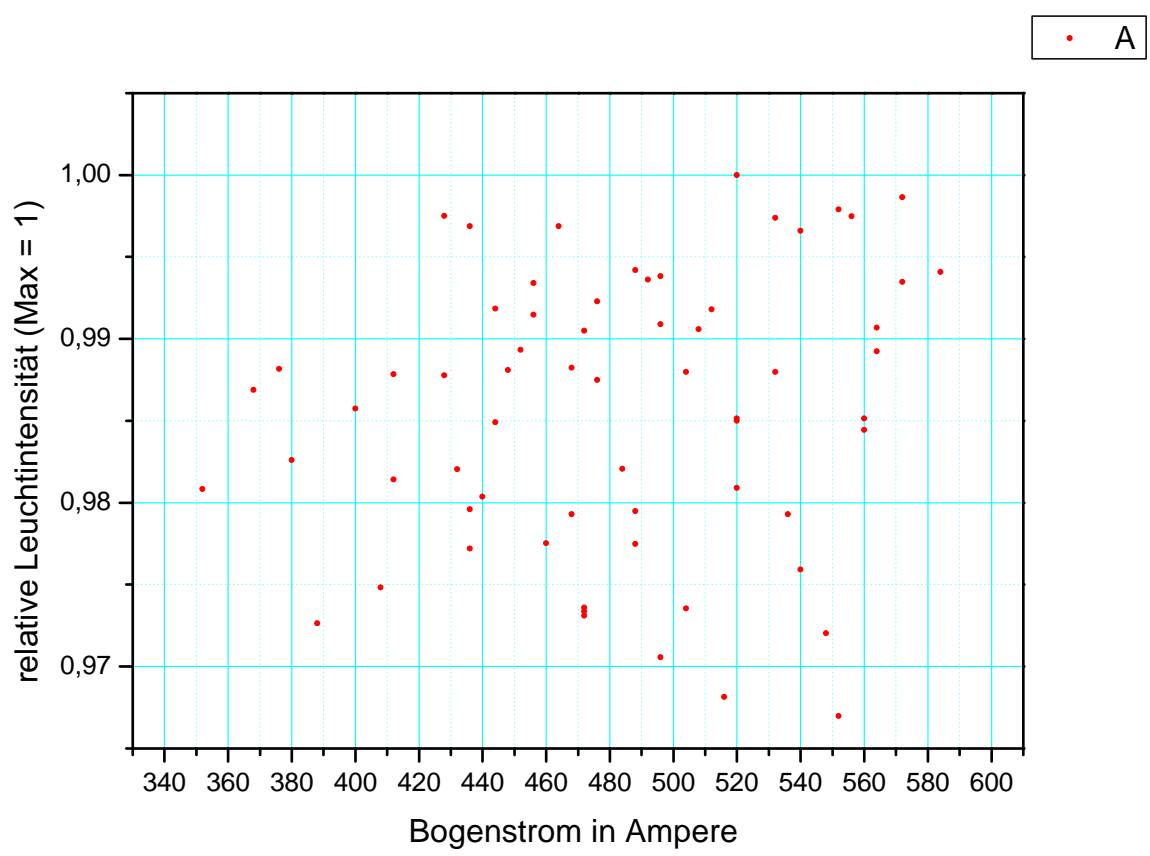

Abbildung 5.44: relative Leuchtintensität der Brennflecken (blau) über den Bogenstrom (rot) auf Kohlenstoff

Die Grafik 5.43 lässt den Eindruck entstehen, dass zwischen Leuchtintensität und Bogen- 
strom eine zeitlichen Verzögerung herrscht. Um dies zu überprüfen, werden beide Größen nicht über der Entladungsdauer sondern gegeneinander Aufgetragen. Abbildung 5.44 zeigt keine Hysterese, was auf eine Verzögerung deuten würde, aber auch keine Gerade.

\subsubsection{Geschwindigkeit der Makropartikel}

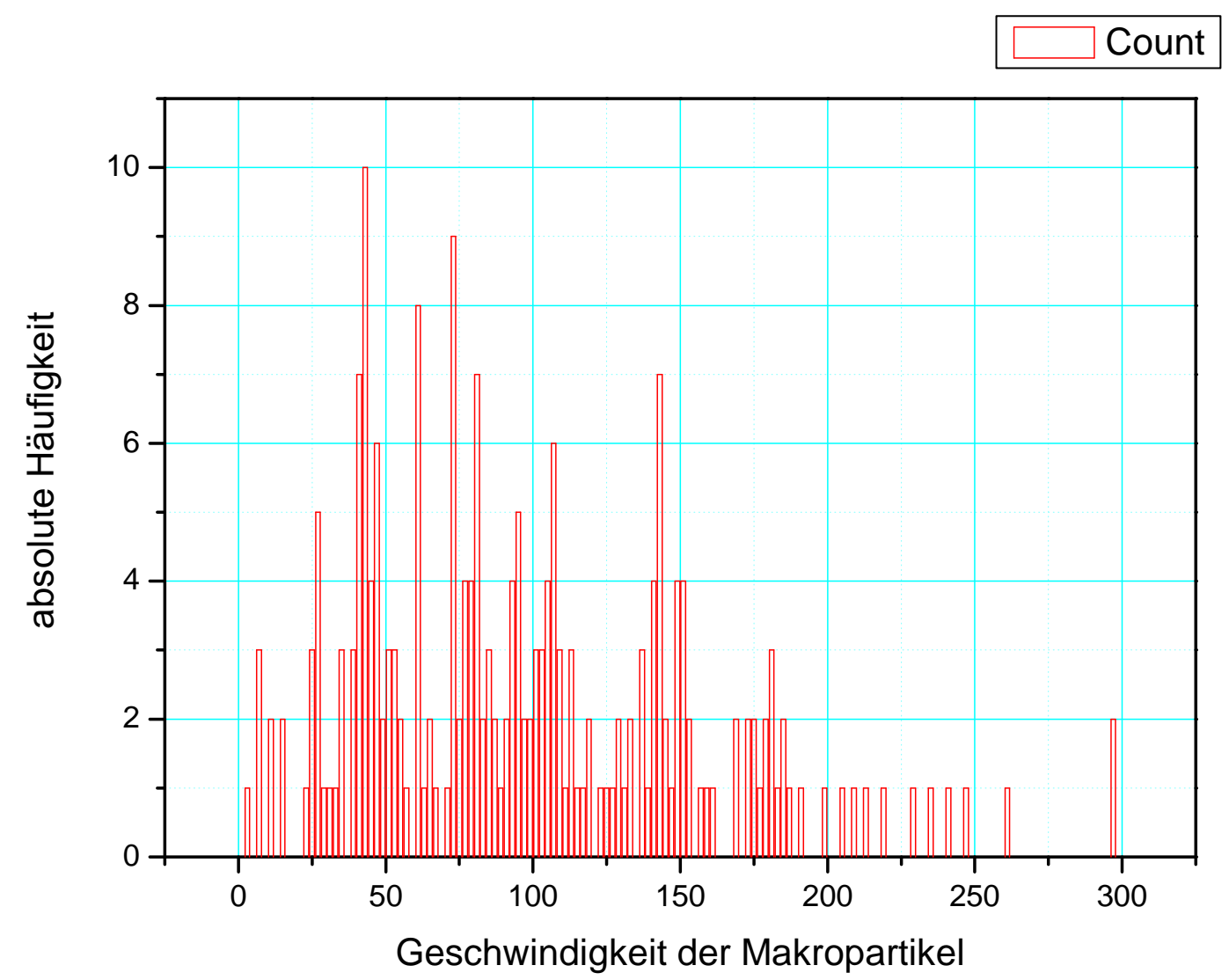

Abbildung 5.45: Verteilung der Makropartikelgeschwindigkeit auf Kohlenstoff; 500 A Bogenstrom, 1 ms Entladungsdauer, Versuchsreihe 101105

Die Geschwindigkeitsmessungen der Makropartikel wurde nach der in Kapitel 4.2.2 auf Seite 28 beschrieben Methode durchgeführt. Abbildung 5.45 gibt die Verteilung der gemessenen Geschwindigkeiten auf Kohlenstoffkathoden an. Die Durchschnittsgeschwindigkeit bzw. die Standardabweichung beträgt $99,8 \mathrm{~m} / \mathrm{s}$ bzw. $60,6 \mathrm{~m} / \mathrm{s}$.

Abbildung 5.46 gibt die Verteilung der gemessenen Geschwindigkeiten auf Wolfram beschichtetem Kohlenstoffkathoden $(\mathrm{CW})$ an. Die Durchschnittsgeschwindigkeit bzw. die Standardabweichung beträgt $26,9 \mathrm{~m} / \mathrm{s}$ bzw. $19,7 \mathrm{~m} / \mathrm{s}$.

Die Makropartikelgeschwindigkeiten sind auf Kohlenstoff breiter verteilt als auf beschichtetem Kohlenstoff. Und deren Durchschnittswerte unterscheiden sich deutlich voneinander. 




Abbildung 5.46: Verteilung der Makropartikelgeschwindigkeit auf Wolfram beschichtetem Kohlenstoff bei 500 A Bogenstrom, 0,5ms Entladungsdauer, Versuchsreihe 101129

\section{Fehlerbetrachtung}

Die Berechnung der Schärfentiefe [20] wird zeigen, dass der Fehler der Geschwindigkeitsmessungen, der aufgrund der Raumtiefenkomponente entsteht, gering ist.

- Für die Kamera mit 4/3-Typ-Digitalsensor (Sensordiagonale d $=22 \mathrm{~mm}$ ) gilt ein Zerstreungskreis Z. $Z=\frac{d}{1500}$

$$
\mathrm{Z}=0,015 \mathrm{~mm}
$$

- Die hyperfokale Länge dh der Anordnung (Objektiv und Kamera) ergibt sich aus Brennweite $\mathrm{f}=60 \mathrm{~mm}$, Blendzahl k (z.B 5,6) und Zerstreungskreis Z. $d h=\frac{f^{2}}{k \cdot Z}+f$

$$
\mathrm{dh}=42 \mathrm{~m}
$$

- Der Abstand von Nahpunkt und Linse dn ergibt sich aus der Geometrie von hyperfokaler Länge dh, Gegenstandsweite g (ca.200 mm) und der Brennweite f. $d n=\frac{g \cdot d h}{d h+(g-f)}$ 


$$
\operatorname{dn}=199,335 \mathrm{~mm}
$$

- Für den Fernpunkt gilt Analoges. $d f=\frac{g \cdot d h}{d h-(g-f)}$

$$
\mathrm{df}=200,669 \mathrm{~mm}
$$

- Die Differenz zwischen Nah- und Fernpunkt wird Schärfentiefe genannt. $\Delta d=d f-d n$

$$
\Delta d 1,34 \mathrm{~mm} \text {. }
$$

Die horizontale und vertikale Ausdehnung der Makropartikelflugbahn ist mit ca. $3 \mathrm{~cm}$ groß in Relation zur Raumtiefenkomponente mit 1,5 mm.

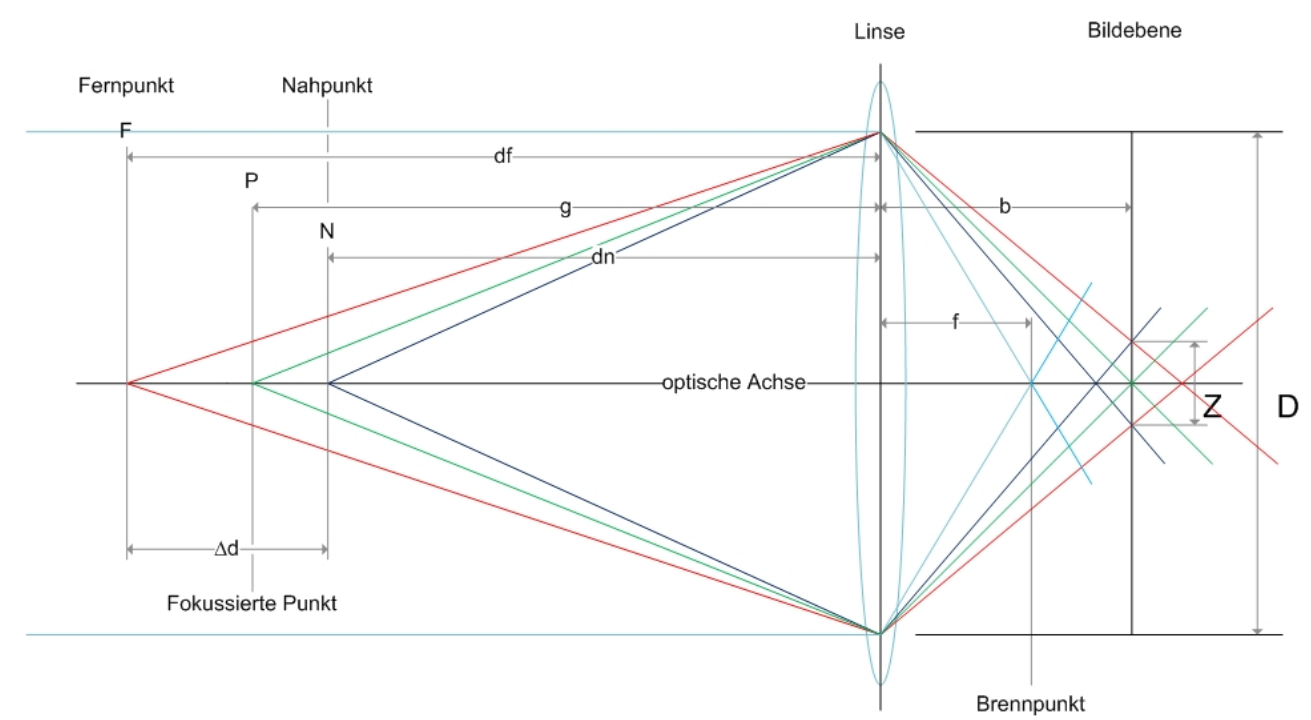

Abbildung 5.47: Schärfentiefe von Nah- bis Fernpunkt $\Delta d$, Linse, Bildebene, optisch Achse, Brennweite $f$, Gegenstandsweite g, Blendendurchmesser D, Zerstreungspunkt Z von [20] 


\section{Kapitel 6}

\section{Zusammenfassung}

Das Ziel der vorliegenden Arbeit war die Gewinnung von reproduzierbaren Laborergebnissen zum Verhalten des Vakuumbogens. Die Ergebnisse werden im weiteren Verlauf der Zusammenarbeit von Arc Precision und dem Max-Planck-Institut für Plasmaphysik ausgewertet, und gegebenenfalls mit der bekannten Literatur verglichen, um Schlussfolgerungen für die Forschung an Fusionsreaktoren zu ziehen, mit dem übergeordneten Ziel die Auswirkungen des Vakuumbogens auf Fusionsreaktoren besser zu verstehen bzw. zu minimieren.

Zu diesem Zweck wurde in Kapitel 2 kurz der derzeitige Wissenstand wiedergegeben. Es wurde ein UHV Pumpstand aufgebaut, in dessen Inneren vielfältige Untersuchungen mit dem Vakuumbogen auf vier unterschiedlichen Kathodenmaterialien durchgeführt werden konnten (siehe Kapitel 3 und Kapitel 4).

Die Vakuumbogenbrennflecken und Makropartikel konnten mit einer Hochgeschwindigkeitskamera beobachtet und sowohl die Kathodenerosion als auch die Makropartikel mikroskopisch untersucht werden. Die Ergebnisse sind in Kapitel 5.1 dokumentiert.

Eine ausführliche Übersicht über die Experimente ist im Anhang zu finden (Tabelle 6.1). Darüber hinaus wurden Proben und das gesamte Filmmaterial an das IPP zur weitergehenden Untersuchung übergeben.

Im Ergebnis der Beobachtungen und Auswertungen wurden folgende allgemeine Beobachtungen gemacht.

- Auf allen Materialien wurden die unterschiedlichen Brennflecktypen dargestellt

- Brennflecken auf Kohlenstoff haben eine deutlich längere Lebenszeit und glühen darüber hinaus auch noch nach der Entladung

- Die Brennflecken brennen bevorzugt auf dekontaminierten Oberflächen (Bevorzugung Typ I vor Typ II bei W, CW und $\mathrm{W} \mathrm{Al}_{2} \mathrm{O}_{3}$ )

- Die Proben C und CW weisen im Gegensatz zu W und W $\mathrm{Al}_{2} \mathrm{O}_{3}$ starke Oberflächenerosion auf (Krater auf der Oberfläche und tiefe Löcher in der Oberfläche)

- Nicht bestätigt werden konnte die Theorie der Bevorzugung von geringen Auswurfwinkeln der Makropartikel aus den Kathodenbrennflecken. Hierzu sind jedoch noch nicht alle Untersuchungen abgeschlossen

- Es konnte keine Einfluss eines externen Magnetfeldes auf die Makropartikel nachgewiesen werden. 
In Kapitel 5.2 wurden vier separate Fragestellungen beantwortet.

1. Die Strukturebenen des Vakuumbogenbrennfleckes wurden analysiert und definiert. Dabei wurde zwischen Bogenspuren, Brennflecken bzw. der Brennfleckfront und Subbrennflecken unterschieden.

2. Es konnte das lineare Ansteigen der Geschwindigkeit der Brennfleckfront, mit steigender Magnetfeldstärke, für den Bereich von $10 \mathrm{mT}$ bis $150 \mathrm{mT}$ auf Kohlenstoff bestätigt werden, und die Geschwindigkeit für alle Brennflecktypen auf allen Materialien gemessen werden.

3. Des weiteren wurde die Abhängigkeit der Leuchtintensität für langsame Kathodenbrennflecken von der Höhe des Bogenstromes für Kohlenstoff und CW N100 gezeigt.

4. Die Geschwindigkeitsverteilung der Makropartikel wurden für Kohlenstoff und beschichteten Kohlenstoff angegeben. 


\section{Anhang}

Tabelle 6.1: Übersicht über das aufgenommene Filmmaterial; Spulenstrom (I $\left.I_{\text {Spule }}\right)$, Bogenstrom (I $\left.I_{\text {Bogen }}\right)$, Bildabstand/Belichtungszeit (exp), Filmaufnahme der Hochgeschwindigkeitskamera (Film), Foto der Übersichtskamera (Foto), Entladungsnummer (Ent. nr.), Entladungsdauer $\left(t_{\text {ent }}\right)$, Entladungsabstand ( $\left.t_{\text {entab }}\right)$ und die Oszillografenaufzeichnung (Osz.)

\begin{tabular}{rrrcccccc}
\multicolumn{10}{c}{$\mathbf{1 0 1 0 2 1}$ - Kohlenstoff } \\
\hline$I_{\text {Spule }}$ & $I_{\text {Bogen }}$ & $\exp$ & Film & Foto & Ent. nr. & $t_{\text {ent }}$ & $t_{\text {entab }}$ & Osz. \\
{$[A]$} & {$[A]$} & {$[\mu s / \mu s]$} & & & & {$[m s]$} & {$[m s]$} & \\
00 & 500 & $27 / 9$ & cine04 & & $61-80$ & 10 & 120 &
\end{tabular}

\begin{tabular}{rrrlrcrrr}
\multicolumn{10}{c}{$\mathbf{1 0 1 0 2 7}$ - Kohlenstoff } \\
\hline$I_{\text {Spule }}$ & $I_{\text {Bogen }}$ & $\exp$ & Film & Foto & Ent. nr. & $t_{\text {ent }}$ & $t_{\text {entab }}$ & Osz. \\
{$[A]$} & {$[A]$} & {$[\mu s / \mu s]$} & & & & {$[m s]$} & {$[m s]$} & \\
500 & 500 & $27 / 25$ & cin04 & & 5 & 5 & 120 & 0607
\end{tabular}

\begin{tabular}{rrrlrcrrr}
\multicolumn{10}{c}{$\mathbf{1 0 1 0 2 8}$ - Kohlenstoff } \\
\hline$I_{\text {Spule }}$ & $I_{\text {Bogen }}$ & exp & Film & Foto & Ent. nr. & $t_{\text {ent }}$ & $t_{\text {entab }}$ & Osz. \\
{$[A]$} & {$[A]$} & {$[\mu s / \mu s]$} & & & {$[m s]$} & {$[m s]$} & \\
500 & 500 & $100 / 98$ & cine01 & 1 & 1 & & 1011 \\
100 & 500 & $100 / 98$ & cine02 & 2 & 1 & 70 & 1213 \\
100 & 500 & $100 / 98$ & cine04 & 4 & 1 & 70 & 1617 \\
100 & 500 & $50 / 9$ & cine05 & 5 & 1 & 70 & 1819 \\
100 & 500 & $50 / 9$ & cine06 & 6 & 1 & 70 & 2021 \\
100 & 500 & $50 / 1$ & cine07 & 7 & 1 & 70 & 24 \\
100 & 500 & $50 / 1$ & cine08 & 8 & 1 & 70 & 25 \\
100 & 800 & $50 / 1$ & cine09 & 9 & 1 & 70 & 2627 \\
100 & 800 & $50 / 1$ & cine10 & 10 & 1 & 70 & 2829 \\
200 & 500 & $50 / 1$ & cine11 & 11 & 3 & & 3031 \\
100 & 500 & $50 / 1$ & cine15 & 15 & 5 & & 5152 \\
100 & 500 & $50 / 1$ & cine16 & 16 & 5 & & 5354 \\
200 & 500 & $50 / 1$ & cine17 & 17 & 5 & & 5556
\end{tabular}

101104 - Kohlenstoff

\begin{tabular}{rrrcccccr}
\hline$I_{\text {Spule }}$ & $I_{\text {Bogen }}$ & $\exp$ & Film & Foto & Ent. nr. & $t_{\text {ent }}$ & $t_{\text {entab }}$ & Osz. \\
{$[A]$} & {$[A]$} & {$[\mu s / \mu s]$} & & & & {$[m s]$} & {$[m s]$} & \\
00 & 500 & $100 / 98$ & cine01 & & 1 & 1 & 70 &
\end{tabular}




$\begin{array}{rrrllll}00 & 500 & 100 / 19 & \text { cine02 } & 2 & 1 & 70 \\ 00 & 500 & 100 / 19 & \text { cine03 } & 3 & 1 & 70 \\ 00 & 500 & 27 / 9 & \text { cine06 } & 6 & 1 & 70\end{array}$

101105 - Kohlenstoff

\begin{tabular}{|c|c|c|c|c|c|c|c|}
\hline $\begin{array}{r}I_{\text {Spule }} \\
{[A]}\end{array}$ & $\begin{array}{r}I_{\text {Bogen }} \\
{[A]}\end{array}$ & $\begin{array}{r}\exp \\
{[\mu s / \mu s]}\end{array}$ & Film & Foto Ent. nr. & $\begin{array}{l}t_{\text {ent }} \\
{[m s]}\end{array}$ & $\begin{array}{c}t_{\text {entab }} \\
\text { [ms] }\end{array}$ & Osz. \\
\hline 00 & 500 & $27 / 9$ & cine07 & & 1 & & 6465 \\
\hline 00 & 500 & $50 / 9$ & cine08 & & 1 & & 6667 \\
\hline 00 & 500 & $50 / 9$ & cine09 & & 1 & & 6869 \\
\hline 00 & 500 & $50 / 9$ & cine10 & & 8 & & 7071 \\
\hline $100 \mathrm{~A}$ & 500 & $50 / 9$ & cine11 & & 5 & & 7273 \\
\hline $100 \mathrm{~A}$ & 500 & $50 / 9$ & cine12 & & 5 & & 7677 \\
\hline $100 \mathrm{~A}$ & 500 & $50 / 9$ & cine13 & & 5 & & 7879 \\
\hline $100 \mathrm{~A}$ & 500 & $50 / 9$ & cine14 & & 5 & & 8081 \\
\hline $100 \mathrm{~A}$ & 500 & $50 / 9$ & cine15 & & 5 & & 8283 \\
\hline $200 \mathrm{~A}$ & 500 & $50 / 9$ & cine16 & & 5 & & 8485 \\
\hline $200 \mathrm{~A}$ & 500 & $50 / 9$ & cine17 & & 5 & & 8687 \\
\hline $200 \mathrm{~A}$ & 500 & $50 / 9$ & cine18 & & 5 & & 8788 \\
\hline $200 \mathrm{~A}$ & 500 & $50 / 9$ & cine19 & & 5 & & 8990 \\
\hline $300 \mathrm{~A}$ & 500 & $50 / 9$ & cine20 & & 2 & & 9293 \\
\hline $300 \mathrm{~A}$ & 500 & $50 / 9$ & cine22 & & 2 & & 9495 \\
\hline $300 \mathrm{~A}$ & 500 & $50 / 9$ & cine23 & & 2 & & 9697 \\
\hline $300 \mathrm{~A}$ & 500 & $50 / 9$ & cine24 & & 2 & & 0001 \\
\hline $400 \mathrm{~A}$ & 500 & $50 / 9$ & cine25 & & 1 & & 0203 \\
\hline $400 \mathrm{~A}$ & 1000 & $50 / 9$ & cine31 & & 2 & & 1415 \\
\hline $200 \mathrm{~A}$ & 1000 & $50 / 9$ & cine33 & & 2 & & 1819 \\
\hline $100 \mathrm{~A}$ & 1000 & $50 / 9$ & cine34 & & 2 & & 2021 \\
\hline $100 \mathrm{~A}$ & 1000 & $50 / 9$ & cine35 & & 2 & & 2224 \\
\hline $100 \mathrm{~A}$ & 1000 & $50 / 9$ & cine36 & & 2 & & 2526 \\
\hline
\end{tabular}

101118 - Wolfram

\begin{tabular}{|c|c|c|c|c|c|c|c|c|}
\hline $\begin{array}{r}I_{\text {Spule }} \\
{[A]}\end{array}$ & $\begin{array}{r}I_{\text {Bogen }} \\
{[A]}\end{array}$ & $\begin{array}{r}\exp \\
{[\mu s / \mu s]}\end{array}$ & Film & Foto & Ent. nr. & $\begin{array}{l}t_{\text {ent }} \\
\text { [ms] }\end{array}$ & $\begin{array}{c}t_{\text {entab }} \\
{[\mathrm{ms}]}\end{array}$ & Osz. \\
\hline $100 \mathrm{~A}$ & 500 & $50 / 19$ & cine01 & & 5 & 2 & & 0,1 \\
\hline $100 \mathrm{~A}$ & 500 & $50 / 1$ & cine04 & & 9 & 2 & & 4,5 \\
\hline 00 & 500 & $50 / 1$ & cine05 & & 10 & 2 & & 5,7 \\
\hline 00 & 500 & $50 / 1$ & cine06 & & 11 & 2 & & 8,9 \\
\hline 00 & 500 & $10 / 2$ & cine08 & & 14 & 2 & & 12,13 \\
\hline 00 & 500 & $10 / 2$ & cine09 & & 15 & 2 & & \\
\hline 00 & 500 & $50 / 1$ & cine10 & & 16 & 2 & & \\
\hline 00 & 500 & $50 / 48$ & cine11 & & 17 & 2 & & 17,18 \\
\hline 00 & 500 & $50 / 48$ & cine12 & & 18 & 2 & & 19,20 \\
\hline 00 & 500 & $50 / 1$ & cine13 & & 19 & 2 & & 21,22 \\
\hline
\end{tabular}


101123 - Wolfram

\begin{tabular}{|c|c|c|c|c|c|c|c|c|}
\hline $\begin{array}{r}I_{\text {Spule }} \\
{[A]}\end{array}$ & $\begin{array}{r}I_{\text {Bogen }} \\
{[A]}\end{array}$ & $\begin{array}{r}\exp \\
{[\mu s / \mu s]}\end{array}$ & Film & Foto & Ent. $n r$. & $\begin{array}{l}t_{\text {ent }} \\
{[m s]}\end{array}$ & $\begin{array}{l}t_{\text {entab }} \\
{[m s]}\end{array}$ & Osz. \\
\hline 00 & 500 & $50 / 9$ & cine02 & 8 & 2 & 0,5 & & 0203 \\
\hline 50 & 500 & $50 / 9$ & cine05 & 9 & 3 & 0,5 & & \\
\hline 50 & 500 & $50 / 9$ & cine05 & 11 & 5 & 0,5 & & 0607 \\
\hline 50 & 500 & $50 / 48$ & cine07 & 12 & 22 & 0,5 & & \\
\hline 50 & 500 & $50 / 48$ & cine08 & 13 & 23 & 0,5 & & \\
\hline 50 & 500 & $50 / 48$ & cine07 & 14 & 24 & 0,5 & & 0813 \\
\hline 50 & 500 & $50 / 1$ & cine10 & 16 & 25 & 0,5 & & 1516 \\
\hline 50 & 500 & $50 / 1$ & cine12 & 18 & 27 & 0,5 & 120 & 1819 \\
\hline 100 & 500 & $50 / 9$ & cine14 & 20 & 28 & 0,5 & 120 & 2226 \\
\hline 100 & 500 & $50 / 9$ & cine15 & 21 & 29 & 0,5 & & \\
\hline 100 & 500 & $50 / 9$ & cine16 & 25 & 30 & 0,5 & & \\
\hline 100 & 500 & $50 / 9$ & cine17 & & $31-35$ & 0,5 & 120 & $27-28$ \\
\hline 00 & 500 & $100 / 38$ & cine18 & & $36-40$ & 0,5 & 120 & \\
\hline 00 & 500 & $100 / 38$ & cine18 & & $41-46$ & 0,5 & 120 & 2930 \\
\hline 50 & 500 & $100 / 38$ & cine20 & & $47-51$ & 0,5 & 120 & 3132 \\
\hline
\end{tabular}

101125 - Wolfram Kohlenstoff

\begin{tabular}{|c|c|c|c|c|c|c|c|c|}
\hline $\begin{array}{c}I_{\text {Spule }} \\
{[\mathrm{A}]}\end{array}$ & $\begin{array}{r}I_{\text {Bogen }} \\
{[\mathrm{A}]}\end{array}$ & $\begin{array}{r}\exp \\
{[\mu s / \mu s]}\end{array}$ & Film & Foto & Ent. $n r$. & $\begin{array}{l}t_{\text {ent }} \\
{[m s]}\end{array}$ & $\begin{array}{l}t_{\text {entab }} \\
{[\mathrm{ms}]}\end{array}$ & Osz. \\
\hline 00 & 500 & $50 / 9$ & cine01 & 5 & 1 & 0,5 & & \\
\hline 00 & 500 & $50 / 9$ & cine02 & 6 & 2 & 0,5 & & \\
\hline 00 & 500 & $50 / 9$ & cine03 & 7 & 3 & 0,5 & & 0102 \\
\hline 00 & 500 & $50 / 9$ & cine01 & 8 & 4 & 0,5 & & 0304 \\
\hline 00 & 500 & $50 / 1$ & cine05 & 10 & 5 & 0,5 & & 0506 \\
\hline 00 & 500 & $27 / 2$ & cine06 & 12 & 6 & 0,5 & & 0708 \\
\hline 00 & 500 & $27 / 2$ & cine07 & 13 & 7 & 0,5 & & 0910 \\
\hline 00 & 500 & $27 / 2$ & cine08 & 17 & $8-12$ & 0,5 & 70 & 1112 \\
\hline 30 & 500 & $27 / 2$ & cine09 & 18 & $13-17$ & 0,5 & 70 & $13-14$ \\
\hline 60 & 500 & $27 / 2$ & cine10 & 30 & $18-27$ & 0,5 & 70 & $15-16$ \\
\hline 60 & 500 & $15 / 1$ & cine11 & 41 & $28-37$ & 0,5 & 70 & $17-18$ \\
\hline 60 & 500 & $17 / 1$ & cine12 & 55 & $38-42$ & 0,5 & 120 & $19-20$ \\
\hline 60 & 500 & $17 / 15$ & cine13 & 61 & $43-47$ & 0,5 & 120 & $21-22$ \\
\hline 00 & 500 & $50 / 9$ & cine14 & 68 & $48-52$ & 0,5 & 120 & $23-24$ \\
\hline 30 & 500 & $50 / 9$ & cine15 & 74 & $53-57$ & 0,5 & 120 & $25-26$ \\
\hline
\end{tabular}

101129 - Wolfram Kohlenstoff

\begin{tabular}{|c|c|c|c|c|c|c|c|c|}
\hline $\begin{array}{r}I_{\text {Spule }} \\
{[A]}\end{array}$ & $\begin{array}{r}I_{\text {Bogen }} \\
{[A]}\end{array}$ & $\begin{array}{r}\exp \\
{[\mu s / \mu s]}\end{array}$ & Film & Foto & Ent. $n r$. & $\begin{array}{l}t_{\text {ent }} \\
{[m s]}\end{array}$ & $\begin{array}{r}t_{\text {entab }} \\
{[m s]}\end{array}$ & Osz. \\
\hline 00 & 500 & $45 / 8$ & cine04 & 8 & 4 & & & $4-5$ \\
\hline 00 & 500 & $45 / 8$ & cine05 & 10 & 5 & 0,5 & & $6-7$ \\
\hline 00 & 500 & $45 / 19$ & cine06 & 12 & 6 & 0,5 & & $8-9$ \\
\hline 30 & 500 & $45 / 19$ & cine07 & 14 & 7 & 0,5 & & \\
\hline 30 & 500 & $45 / 19$ & cine08 & 16 & 8 & 0,5 & & $0-11$ \\
\hline 30 & 500 & $45 / 19$ & cine09 & 18 & 9 & 0,5 & & $2-13$ \\
\hline
\end{tabular}




$\begin{array}{rrrlcccrr}30 & 500 & 100 / 39 & \text { cine11 } & 22 & 11 & 0,5 & & \\ 30 & 500 & 45 / 19 & \text { cine10 } & 20 & 10 & 0,5 & & 14-15 \\ 30 & 500 & 100 / 39 & \text { cine12 } & 26 & 31-35 & 0,5 & 120 & 16-17 \\ 30 & 500 & 100 / 39 & \text { cine13 } & 39 & 36-40 & 0,5 & 120 & 18-19 \\ 30 & 500 & 100 / 39 & \text { cine15 } & 53 & 91-95 & 0,5 & 120 & 22 \\ 30 & 500 & 100 / 39 & \text { cine16 } & 60 & 196-200 & 0,5 & 120 & 23-24 \\ 30 & 500 & 100 / 39 & \text { cine17 } & 66 & 201-205 & 0,5 & 120 & 25-26 \\ 60 & 500 & 100 / 19 & \text { cine18 } & 68 & 206-410 & 0,5 & 120 & 27-28 \\ 60 & 500 & 100 / 9 & \text { cine19 } & 70 & 411-415 & 0,5 & 120 & \end{array}$

101201 - Wolfram Kohlenstoff

\begin{tabular}{|c|c|c|c|c|c|c|c|c|}
\hline $\begin{array}{r}I_{\text {Spule }} \\
{[A]}\end{array}$ & $\begin{array}{r}I_{\text {Bogen }} \\
{[A]}\end{array}$ & $\begin{array}{r}\exp \\
{[\mu s / \mu s]}\end{array}$ & Film & Foto & Ent. nr. & $\begin{array}{l}t_{\text {ent }} \\
{[\mathrm{ms}]}\end{array}$ & $\begin{array}{l}t_{\text {entab }} \\
{[\mathrm{ms}]}\end{array}$ & Osz. \\
\hline 00 & 500 & $91 / 19$ & cine01 & 4 & 1 & 0,5 & & $00-01$ \\
\hline 00 & 500 & $91 / 1$ & cine02 & 6 & 2 & 0,5 & & $2-3$ \\
\hline 00 & 500 & $25 / 1$ & cine03 & 11 & 3 & 0,5 & & $4-5$ \\
\hline 00 & 500 & $25 / 1$ & cine04 & 14 & 5 & 0,5 & & $7-8$ \\
\hline 30 & 500 & $25 / 1$ & cine05 & 15 & 6 & 0,5 & & $9-10$ \\
\hline 30 & 500 & $25 / 1$ & cine06 & 18 & 7 & 0,5 & & $11-12$ \\
\hline 30 & 500 & $16 / 1$ & cine07 & 20 & 8 & 0,5 & & $13-14$ \\
\hline 60 & 500 & $25 / 1$ & cine08 & 22 & 9 & 0,5 & & $15-16$ \\
\hline
\end{tabular}

$101223-W \mathrm{Al}_{2} \mathrm{O}_{3}$

\begin{tabular}{|c|c|c|c|c|c|c|c|c|}
\hline $\begin{array}{r}I_{\text {Spule }} \\
{[A]}\end{array}$ & $\begin{array}{r}I_{\text {Bogen }} \\
{[A]}\end{array}$ & $\begin{array}{r}\exp \\
{[\mu s / \mu s]}\end{array}$ & Film & Foto & Ent. $n r$ & $\begin{array}{l}t_{\text {ent }} \\
{[m s]}\end{array}$ & $\begin{array}{c}t_{\text {entab }} \\
{[\mathrm{ms}]}\end{array}$ & Osz. \\
\hline 00 & 500 & $100 / 98$ & cine01 & $1708 / 09$ & 3 & 0,5 & & 0607 \\
\hline 00 & 500 & $58 / 55$ & cine02 & $1710 / 11$ & 4 & 0,5 & & 0809 \\
\hline 00 & 500 & $58 / 55$ & cine03 & 1712 & 5 & 0,5 & & 1011 \\
\hline 00 & 500 & $58 / 55$ & cine04 & 1713 & $6-10$ & 0,5 & 70 & 1213 \\
\hline 00 & 500 & $58 / 2$ & cine05 & 1714 & $11-15$ & 0,5 & 70 & 1415 \\
\hline 00 & 500 & $58 / 10$ & cine06 & $1715 / 16$ & $16-20$ & 0,5 & 70 & 1617 \\
\hline
\end{tabular}

$101228-W \mathrm{Al}_{2} \mathrm{O}_{3}$

\begin{tabular}{|c|c|c|c|c|c|c|c|c|}
\hline $\begin{array}{r}I_{\text {Spule }} \\
{[A]}\end{array}$ & $\begin{array}{r}I_{\text {Bogen }} \\
{[A]}\end{array}$ & $\begin{array}{r}\exp \\
{[\mu s / \mu s]}\end{array}$ & Film & Foto & Ent. $n r$. & $\begin{array}{l}t_{\text {ent }} \\
\text { [ms] }\end{array}$ & $\begin{array}{r}t_{\text {entab }} \\
{[\mathrm{ms}]}\end{array}$ & Osz. \\
\hline 00 & 500 & $56 / 56$ & cine01 & 1717 & 1 & 0,5 & & 0001 \\
\hline 00 & 500 & $35 / 35$ & cine02 & 1719 & 3 & 0,5 & & 0405 \\
\hline 00 & 500 & $35 / 35$ & cine03 & 1720 & 4 & 0,5 & & 0607 \\
\hline 100 & 500 & $56 / 56$ & cine04 & 1722 & 5 & 0,5 & & 0809 \\
\hline 100 & 500 & $60 / 10$ & cine05 & 1723 & 6 & 0,5 & & 1011 \\
\hline 100 & 500 & $60 / 2$ & cine06 & 1724 & 7 & 0,5 & & 1213 \\
\hline 50 & 500 & $60 / 2$ & cine07 & 1725 & 8 & 0,5 & & 1415 \\
\hline 50 & 500 & $74 / 2$ & cine08 & 1726 & 9 & 0,5 & & 1617 \\
\hline 50 & 500 & $74 / 2$ & cine09 & 1727 & $10-12$ & 0,5 & 70 & 1819 \\
\hline 100 & 500 & $56 / 56$ & cine10 & 1728 & $13-15$ & 0,5 & 70 & 2021 \\
\hline 100 & 500 & $53 / 2$ & cine11 & 1729 & $16-17$ & 0,5 & 70 & 2223 \\
\hline 100 & 500 & $53 / 20$ & cine12 & 1730 & $18-19$ & 0,5 & 70 & 2425 \\
\hline
\end{tabular}


110105 - Wolfram Kohlenstoff

\begin{tabular}{rrrcccccr}
\hline$I_{\text {Spule }}$ & $I_{\text {Bogen }}$ & exp & Film & Foto & Ent. nr. & $t_{\text {ent }}$ & $t_{\text {entab }}$ & Osz. \\
{$[A]$} & {$[A]$} & {$[\mu s / \mu s]$} & & & & {$[m s]$} & {$[m s]$} & \\
00 & 500 & $140 / 90$ & cine01 & 1742 & 1 & 0,5 & & 0001 \\
00 & 500 & $140 / 90$ & cine02 & 1743 & 2 & Fehlz & & 0203 \\
00 & 500 & $140 / 140$ & cine03 & 1744 & 3 & Fehlz & & 04
\end{tabular}

\section{7 - Wolfram Kohlenstoff}

\begin{tabular}{rrrcccccr}
\hline$I_{\text {Spule }}$ & $I_{\text {Bogen }}$ & exp & Film & Foto & Ent. $n r$. & $\begin{array}{c}t_{\text {ent }} \\
{[\mathrm{ms}]}\end{array}$ & $\begin{array}{c}t_{\text {entab }} \\
{[\mathrm{ms}]}\end{array}$ & Osz. \\
\hline 00 & 500 & $140 / 140$ & & & & & & \\
00 & 500 & $140 / 140$ & cine01 & 1 & 1 & 0,5 & & 0001 \\
00 & 500 & $43 / 2$ & cine02 & 2 & 2 & 0,5 & & 0203 \\
00 & 500 & $43 / 2$ & cine03 & 3 & 3 & 0,5 & & 0405 \\
00 & 500 & $43 / 2$ & cine04 & 4 & 4 & 0,5 & & 0607 \\
00 & 500 & $43 / 2$ & cine05 & 5 & 5 & 0,5 & & 0809 \\
00 & 500 & $43 / 2$ & cine06 & 6 & 6 & 0,5 & & 1011 \\
00 & 500 & $43 / 43$ & cine07 & $7-10$ & $7-10$ & 0,3 & 70 & 1213 \\
00 & 500 & $43 / 43$ & cine08 & $11-14$ & $11-14$ & 0,3 & 70 & 1415 \\
00 & 500 & $43 / 10$ & cine09 & $15-20$ & $15-20$ & 0,3 & 70 & 1617 \\
00 & 500 & $98 / 50$ & cine10 & $21-30$ & $21-30$ & 0,3 & 70 & 18 \\
00 & 500 & $98 / 50$ & cine11 & $31-40$ & $31-40$ & 0,3 & 70 & 1920 \\
00 & 500 & $98 / 50$ & cine12 & $41-51$ & $41-51$ & 0,3 & 70 & 2122
\end{tabular}

110113 - Wolfram Kohlenstoff

\begin{tabular}{|c|c|c|c|c|c|c|c|c|}
\hline $\begin{array}{r}I_{\text {Spule }} \\
{[A]}\end{array}$ & $\begin{array}{r}I_{\text {Bogen }} \\
{[A]}\end{array}$ & $\begin{array}{r}\exp \\
{[\mu s / \mu s]}\end{array}$ & Film & Foto & Ent. nr. & $\begin{array}{l}t_{\text {ent }} \\
{[\mathrm{ms}]}\end{array}$ & $\begin{array}{l}t_{\text {entab }} \\
\text { [ms] }\end{array}$ & Osz. \\
\hline 00 & 500 & $18 / 2$ & cine01 & 001 & 1 & 0,3 & & 0001 \\
\hline 00 & 500 & $11 / 1$ & cine02 & 002 & 2 & 0,3 & & 0203 \\
\hline 00 & 500 & $11 / 5$ & cine03 & 003 & 3 & 0,3 & & 0405 \\
\hline 00 & 500 & $11 / 9$ & cine04 & 004 & 4 & 0,3 & & 0607 \\
\hline 00 & 500 & $25 / 9$ & cine05 & $5-7$ & $5-7$ & 0,3 & 120 & 0809 \\
\hline 00 & 500 & $66 / 63$ & cine06 & $8-10$ & $8-10$ & 0,3 & 120 & 1011 \\
\hline 00 & 500 & $66 / 63$ & cine07 & $16-19$ & $16-20$ & 0,3 & 120 & 1213 \\
\hline 00 & 500 & $66 / 63$ & cine08 & 20 & $21-25$ & 0,3 & 120 & 1415 \\
\hline 00 & 500 & $66 / 63$ & cine09 & 50 & $51-55$ & 0,3 & 120 & 1617 \\
\hline 00 & 500 & $66 / 63$ & cine10 & 100 & $101-105$ & 0,3 & 120 & $18-19$ \\
\hline 00 & 500 & $66 / 63$ & cine11 & $494-499$ & $496-500$ & 0,3 & 120 & 2021 \\
\hline
\end{tabular}

110119 - Wolfram Kohlenstoff

$\begin{array}{rrrcccccr}I_{\text {Spule }} & I_{\text {Bogen }} & \text { exp } & \text { Film } & \text { Foto } & \text { Ent. nr. } & t_{\text {ent }} & t_{\text {entab }} & \text { Osz. } \\ {[A]} & {[A]} & {[\mu s / \mu s]} & & & & {[m s]} & {[m s]} & \\ 00 & 500 & 8 / 1 & \text { cine02 } & 07 & 2 & 0,3 & & \\ 00 & 500 & 8 / 1 & \text { cine03 } & 09 & 3 & 0,3 & & \\ 00 & 500 & 8 / 1 & \text { cine04 } & 12 & 4 & 0,3 & 05-06 \\ 00 & 500 & 35 / 1 & \text { cine05 } & 14 & 201 & 0,3 & 08-09\end{array}$


$\begin{array}{lllllllll}00 & 500 & 12 / 1 & \text { cine06 } & 15 & 202-204 & 0,3 & 70 \mathrm{~ms} & 09-10\end{array}$

110124 - Kohlenstoff

\begin{tabular}{|c|c|c|c|c|c|c|c|c|}
\hline $\begin{array}{r}I_{\text {Spule }} \\
{[A]}\end{array}$ & $\begin{array}{r}I_{\text {Bogen }} \\
{[\mathrm{A}]}\end{array}$ & $\begin{array}{r}\exp \\
{[\mu s / \mu s]}\end{array}$ & Film & Foto & Ent. $n r$. & $\begin{array}{l}t_{\text {ent }} \\
{[m s]}\end{array}$ & $\begin{array}{l}t_{\text {entab }} \\
{[\mathrm{ms}]}\end{array}$ & Osz. \\
\hline 00 & 500 & $27 / 1$ & cine01 & 02 & $(108-299)$ & 1 & & 01-02 \\
\hline 00 & 500 & $20 / 1$ & cine02 & 03 & 300 & 1 & & 03-05 \\
\hline 00 & 500 & $10 / 1$ & cine03 & 04 & 301 & 2 & & 04-06 \\
\hline 00 & 500 & $10 / 1$ & cine04 & 05 & 302 & 2 & & 06-07 \\
\hline 00 & 3000 & $140 / 1$ & cine05 & 06 & 303 & 1 & & 08-09 \\
\hline 00 & 3000 & $56 / 56$ & cine06 & 07 & 304 & 1 & & $10-11$ \\
\hline 00 & 3000 & $56 / 1$ & cine07 & 08 & 305 & 1 & & $12-13$ \\
\hline 00 & 1000 & $56 / 1$ & cine08 & 09 & 306 & 3 & & $14-15$ \\
\hline 00 & 1000 & $56 / 1$ & cine09 & 10 & 307 & 6 & & $16-17$ \\
\hline 00 & 1000 & $56 / 1$ & cine10 & 11 & 308 & 6 & & 18-19 \\
\hline 50 & 500 & $45 / 2$ & cine11 & 12 & 309 & 5 & & $20-21$ \\
\hline 50 & 500 & $45 / 2$ & cine12 & 13 & 310 & 5 & & $22-23$ \\
\hline 100 & 500 & $45 / 2$ & cine13 & 14 & 311 & 5 & & $24-25$ \\
\hline 100 & 500 & $45 / 2$ & cine14 & & 312 & 1 & & $26-27$ \\
\hline 100 & 500 & $45 / 2$ & cine15 & 15 & 313 & 1 & & $28-29$ \\
\hline 150 & 500 & $45 / 2$ & cine16 & 16 & 314 & 1 & & $32-33$ \\
\hline 150 & 500 & $45 / 2$ & cine17 & 17 & 315 & 1 & & $34-35$ \\
\hline 150 & 500 & $45 / 2$ & cine18 & 20 & 316 & 1 & & $36-37$ \\
\hline 150 & 500 & $45 / 2$ & cine19 & 21 & 317 & 1 & & 38-39 \\
\hline 200 & 500 & $35 / 2$ & cine20 & 22 & 318 & 1 & & $40-41$ \\
\hline 200 & 500 & $35 / 2$ & cine21 & 23 & 319 & 1 & & $41-42$ \\
\hline 250 & 500 & $35 / 2$ & cine 22 & 24 & 320 & 1 & & $43-44$ \\
\hline 250 & 500 & $35 / 2$ & cine23 & 25 & 321 & 0,5 & & $45-46$ \\
\hline 300 & 500 & $35 / 2$ & cine24 & 26 & 322 & 0,5 & & $47-48$ \\
\hline 350 & 500 & $35 / 2$ & cine25 & 27 & 323 & 0,5 & & $49-50$ \\
\hline 400 & 500 & $35 / 2$ & cine26 & & 324 & 0,5 & & $51-52$ \\
\hline 450 & 500 & $44 / 1$ & cine27 & & 325 & 0,5 & & $53-54$ \\
\hline 500 & 500 & $44 / 1$ & cine28 & & 326 & 0,5 & & $55-56$ \\
\hline 600 & 500 & $44 / 1$ & cine29 & & 327 & 0,5 & & $57-58$ \\
\hline 600 & 500 & $44 / 1$ & cine30 & & 328 & 0,5 & & 59-60 \\
\hline 500 & 500 & $44 / 1$ & cine31 & & 329 & 0,5 & & $61-62$ \\
\hline 500 & 500 & $44 / 1$ & cine32 & & $330-334$ & 0,5 & 120 & $63-64$ \\
\hline 500 & $1 \mathrm{kA}$ & $44 / 1$ & cine33 & & $335-339$ & 0,5 & 120 & $65-66$ \\
\hline 200 & $1 \mathrm{kA}$ & $53 / 2$ & cine34 & & $340-344$ & 0,5 & 120 & 67 \\
\hline 200 & $2 \mathrm{kA}$ & $53 / 2$ & cine35 & & $345-349$ & 0,5 & 120 & 68 \\
\hline 200 & 500 & $53 / 2$ & cine36 & & $350-354$ & 0,5 & 120 & $69-70$ \\
\hline 100 & 500 & $53 / 2$ & cine37 & & $355-359$ & 1 & 120 & $71-72$ \\
\hline
\end{tabular}

110125 - Kohlenstoff

\begin{tabular}{rrrcccccr}
\hline$I_{\text {Spule }}$ & $I_{\text {Bogen }}$ & exp & Film & Foto & Ent. nr. & $t_{\text {ent }}$ & $t_{\text {entab }}$ & Osz. \\
{$[A]$} & {$[A]$} & {$[\mu s / \mu s]$} & & & & {$[m s]$} & {$[m s]$} & \\
100 & 500 & $53 / 2$ & cine38 & 2 & 360 & 1 & & $01-02$
\end{tabular}




$\begin{array}{rrrlrcccc}100 & 500 & 53 / 2 & \text { cine39 } & 3 & 361 & 1 & & 03-05 \\ 100 & 500 & 28 / 2 & \text { cine40 } & 4 & 362 & 1 & & 04-06 \\ 100 & 500 & 28 / 2 & \text { cine41 } & 5 & 363 & 1 & & 06-07 \\ 100 & 500 & 28 / 2 & \text { cine42 } & 6 & 364-366 & 1 & 120 & 08-09 \\ 100 & 500 & 28 / 2 & \text { cine43 } & 7 & 367-369 & 1 & 120 & 10-11 \\ 50 & 500 & 28 / 2 & \text { cine44 } & 8 & 370-372 & 2 & 120 & 12-13 \\ 50 & 500 & 28 / 2 & \text { cine45 } & 9 & 373-375 & 5 & 120 & 14-15 \\ 200 & 500 & 28 / 2 & \text { cine46 } & 10 & 376-378 & 0,3 & 120 & 16-17 \\ 200 & 500 & 28 / 2 & \text { cine47 } & 11 & 379-381 & 0,6 & 120 & 18-19 \\ 500 & 500 & 28 / 2 & \text { cine48 } & 12 & 382-384 & 0,3 & 120 & 20-21 \\ 600 & 500 & 28 / 2 & \text { cine49 } & 13 & 385-387 & 0,3 & 120 & 22-23 \\ 700 & 500 & 28 / 2 & \text { cine50 } & 14 & 388-390 & 0,3 & 120 & 24-25 \\ 800 & 500 & 28 / 2 & \text { cine51 } & 15 & 391-393 & 0,3 & 120 & 26-27 \\ 500 & 3000 & 28 / 2 & \text { cine52 } & 16 & 394-396 & 0,3 & 120 & 28-29\end{array}$

110126 -Wolfram

\begin{tabular}{rrrcccccc}
\hline$I_{\text {Spule }}$ & $I_{\text {Bogen }}$ & exp & Film & Foto & Ent. nr. & $t_{\text {ent }}$ & $t_{\text {entab }}$ & Osz. \\
{$[A]$} & {$[A]$} & {$[\mu s / \mu s]$} & & & & {$[m s]$} & {$[m s]$} & \\
00 & 500 & $21 / 20$ & cine01 & 04 & 1 & 0,5 & & 30,31 \\
00 & 500 & $21 / 2$ & cine02 & 05 & 2 & 0,5 & & 32,33 \\
00 & 500 & $7 / 2$ & cine03 & 06 & 3 & 0,5 & 34,35
\end{tabular}

110127 - Wolfram

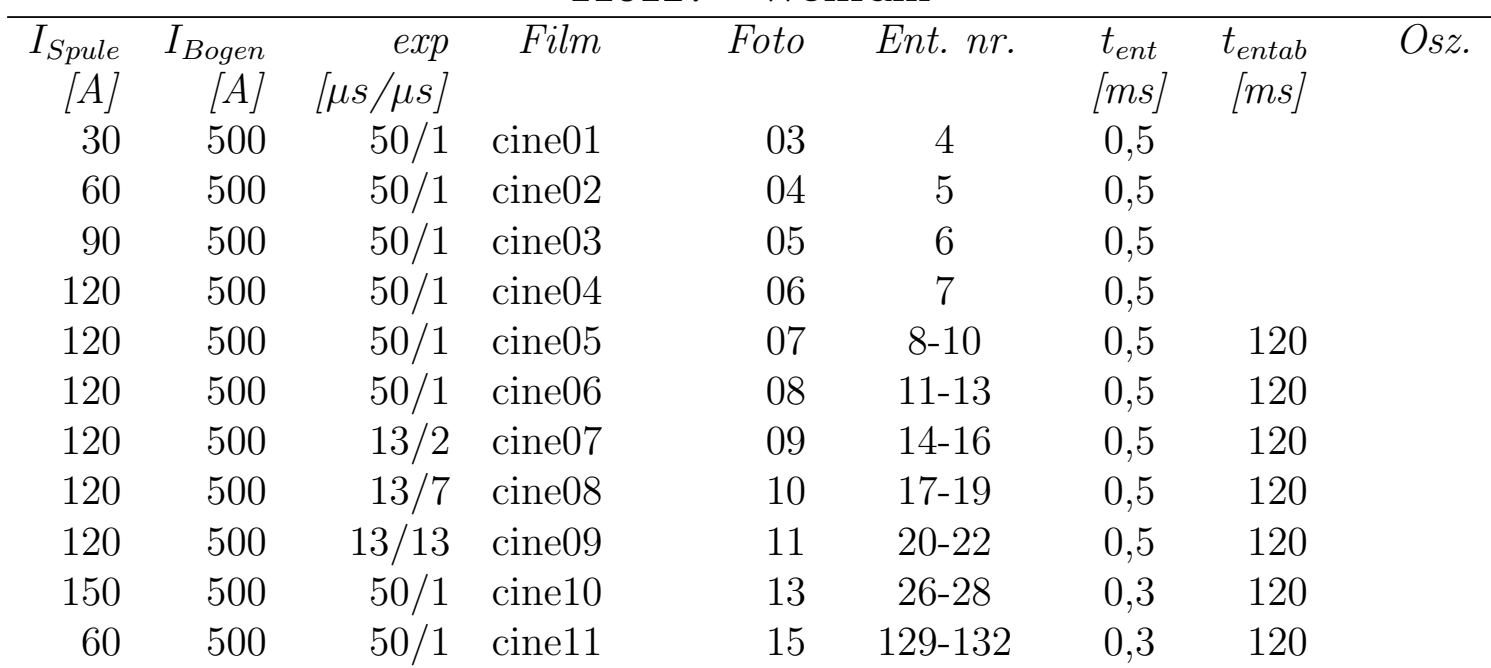

Weitere nicht zitierte Literatur: 


\section{Literaturverzeichnis}

[1] Arc Precision. Angebot zum Forschungsauftrag IPP. Juli 2010.

[2] R. L. Boxman and D. M. Sanders et al. Handbook of Vacuum Arc Science and Technology. Noyes Publication, 1995.

[3] E. Hantzsche. The Thermo-Field Emisiion of Electrons in Arc Discharges. Zentralinstitut für ElektronenPhysik der AdW der DDR, pages 325-346, March 1981.

[4] H. O. Schrade. The arc cathode spot: Their Mechanism and Motion. IEEE Trans. On Plasma Science, 5:635-637, Oktober 1989.

[5] P. J. Martin und D. R MCKenzie et al. Characteristics of Titanium Arc evaporation processes. Thin Solid Films, 153:91-102, 1987.

[6] C. W. Kimblin. Erosion and ionization in the cathode spot regions of vacuum arc. J. Appl. Phys., 44:3079-3081, 1973.

[7] K. Jakubka and B. Jüttner. On the influence of surface conditions on initiation and spot types of unipolar arcs in a Tokamak. J. of Nuc. Mat., 102:259-266, 1981.

[8] H. Wroe. Vaccum Arcs on Tungsten Cathodes. Natur, 182:338, August 1958.

[9] J. E. Daalder. Components of cathode erosion in vacuum arc. J. Phys. D: Appl. Phys., 9:2379 - 2395, 1976.

[10] J. Achtert and B. Altrichter et al. Influence of Surface Contaminations on Cathode Processes of Vacuum Discharges. Beiträge aus der Plasma Physik, 17:419-431, 1977.

[11] A. I. Bushik and B. Jüttner et al. Dynamics of Cathode Processes on a Bimetall Electrode under Ultrahigh Vacuum. High Temperature, 18:555-560, 1980.

[12] N. E. Perskii and V. I. Sysun et al. Dynamics of vacuum-discharge cathode spot. High Temperatur, 27:832-839, 1989.

[13] I. Kleberg. Die Dynamik von kathodischen Brennflecken in externen Magnetfeldern. PhD thesis, Mathematisch-Naturwissenschaftliche Fakultät I der HumboldtUniversität Berlin, 2001.

[14] B. E. Djakov. Cathde spot division in vacuum arcs with solid metal cathodes. J. Phys., 4:504-509, 1971.

[15] J. C. Sherman and Webster et al. Cathode spot motion in high-current vacuum arcs on copper electrodes. J. Phys. D: Appl. Phys., 8:696-702, 1975. 
[16] E. A. Litvinov and G. A. Mesyats et al. On the effect of an external magnetic field on the motion of a vacuum arc cathode spot. Proc. XIVth Int. Symp. Disch. Electr. Insul. Vaccum, pages 185-186, 1990.

[17] M. J. Lafferty. Triggered Vacuum Gaps. Proc. IEEE, 54:23-32, January 1966.

[18] Wikipedia. Zylinderspule — wikipedia, die freie enzyklopädie, 2010. [Online; Stand 16. März 2011].

[19] S. K. Sethuraman et al. . J. Nucl. Matter., 111 und 112:510-516, 1982.

[20] Wikipedia. Schärfentiefe — wikipedia, die freie enzyklopädie, 2011. [Online; Stand 18. März 2011].

[21] R. Basharov and E. M. Gavrilovskaya et al. Sov. Phys. - Tech. Phys., 12:1383-1390, 1967.

[22] J. E. Daalder. Cathoden erosion of metal vapour arcs in vacuum. PhD thesis, Technische Hochschule Eindhoven, 1978.

[23] J. E. Daalder. Random walk of arc cathode spots in vacuum. J. Phys. D: Appl. Phys., 16:17-27, 1983.

[24] J. P. Datlov and A.E. Guile et al. Beiträge aus der Plasma Physik, 21:135-141, 1978.

[25] M. G. Drouet and S. Gruber. Dynamic Measurements of catodic emission in a moving arc. IEEE Trans. Power App. Syst., Pas-95:105-112, January/February 1976.

[26] G. A. Farrel. Low Voltage Firing Characteristics of a Triggered Vacuum Gap. IEEE Trans. Eletrc. Dev., 13:432, 1966.

[27] A. E. Guile and B. Jüttner. Basic Erosion Processes of Oxidized and Clean Metal Cathodes by Electric Arcs. IEEE Transactions on Plasma sciense, 3:259-269, September 1980 .

[28] E. Hantzsche. Beitr. Plasma Phys., 17:65-74, 1977.

[29] E. Hantzsche and B. Jüttner et al. On the random walk of arc cathode spots in vacuum. J. Phys. D: Appl. Phys., 16:173-177, 1983.

[30] E. Hettkamp. Magnetische Beeinflussung von Pulsprozessen bei Vakuumbogenentladung. PhD thesis, Fakultät für Elektrotechnik und Informationstechnik der Otto von Guericke-Universität Magdeburg, 2006.

[31] B. Jüttner. Erosion crater and arc cathodes spots in Vaccum. Beitr. Plasma Phys., 19:25-48, 1979.

[32] T. Klein. Untersuchung der Kraterbildung auf Metallkathoden beim Lichtbogen. PhD thesis, Institut für theoretische Physik der Technischen Universität Braunschweig, 1992.

[33] I. G. Kesaev. Laws govering the cathode drop and the threshold currents in an arc discharge on pure metals. Sov. Phys. - Tech. Phys., 9:1146-1154, 1965. 
[34] R. N. Nilsson. Proceedings of the fourth international conference on Ionization Phenomena in Gases, volume 1. North-Holland Publishing Company - Amsterdam, 1959.

[35] V. F. Puchkarev and A. M. Murzakayev. Current density and the cathode spot lifetime in a vacuum arc at threshold currents. J. Phys. D: Appl. Phys., 23:26-35, 1990.

[36] S. Shalev and S. Goldsmith at al. In Situ Determination of Macroparticle Velocities in a Copper Vacuum Arc. IEEE, Trans. On Plasma Science., 11:146-151, September 1983. 


\section{Danksagung}

An dieser Stelle möchte ich mich bei allen Personen bedanken, die mich bei der Erstellung dieser Arbeit unterstützt haben und zu ihrem guten Gelingen beigetragen haben.

Allen voran den Mitarbeitern von Arc Precision, die mich an ihrer freundlichen und vertrausvollen Arbeitsatmosphäre teil haben ließen. Ein besonderer Dank gilt Dr. rer. nat. P. Siemroth, der mit guten Ideen und sehr viel Geduld meine Diplomarbeit betreute.

Ebenfalls bedanken möchte ich mich bei meinem Hochschulbetreuer Dipl. Phys. H. Beyer, der mich bei Arc Precision vorstellte und stets für mich ansprechbar war.

Bedanken möchte ich mich bei meiner Familie, die mir nicht nur das Studium ermöglichte sondern mich auch dazu motivierte.

Großer Dank gebührt auch meinen Freunden, ohne die die letzten vier Jahre nicht so großartig gewesen wären. 